\title{
Patterns of flavour violation in models with vector-like quarks
}

\author{
Christoph Bobeth, ${ }^{a, b, d}$ Andrzej J. Buras, ${ }^{a, b}$ Alejandro Celis ${ }^{c}$ and Martin Jung ${ }^{a, d}$ \\ ${ }^{a}$ TUM Institute for Advanced Study, Lichtenbergstr. 2a, D-85748 Garching, Germany \\ ${ }^{b}$ Physik Department, TU München, James-Franck-Straße, D-85748 Garching, Germany \\ ${ }^{c}$ Ludwig-Maximilians-Universität München, Fakultät für Physik, \\ Arnold Sommerfeld Center for Theoretical Physics, 80333 München, Germany \\ ${ }^{d}$ Excellence Cluster Universe, Technische Universität München, \\ Boltzmannstr. 2, D-85748 Garching, Germany \\ E-mail: christoph.bobeth@ph.tum.de, aburas@ph.tum.de, \\ Alejandro.Celis@physik.uni-muenchen.de, martin.jung@tum.de
}

ABSTRACT: We study the patterns of flavour violation in renormalisable extensions of the Standard Model (SM) that contain vector-like quarks (VLQs) in a single complex representation of either the SM gauge group $\mathrm{G}_{\mathrm{SM}}$ or $\mathrm{G}_{\mathrm{SM}}^{\prime} \equiv \mathrm{G}_{\mathrm{SM}} \otimes \mathrm{U}(1)_{\mathrm{L}_{\mu}-\mathrm{L}_{\tau}}$. We first decouple VLQs in the $M=(1-10) \mathrm{TeV}$ range and then at the electroweak scale also $Z, Z^{\prime}$ gauge bosons and additional scalars to study the phenomenology. The results depend on the relative size of $Z$ - and $Z^{\prime}$-induced flavour-changing neutral currents, as well as the size of $|\Delta F|=2$ contributions including the effects of renormalisation group Yukawa evolution from $M$ to the electroweak scale that turn out to be very important for models with right-handed currents through the generation of left-right operators. In addition to rare decays like $P \rightarrow \ell \bar{\ell}, P \rightarrow P^{\prime} \ell \bar{\ell}, P \rightarrow P^{\prime} \nu \bar{\nu}$ with $P=K, B_{s}, B_{d}$ and $|\Delta F|=2$ observables we analyze the ratio $\varepsilon^{\prime} / \varepsilon$ which appears in the SM to be significantly below the data. We study patterns and correlations between these observables which taken together should in the future allow for differentiating between VLQ models. In particular the patterns in models with left-handed and right-handed currents are markedly different from each other. Among the highlights are large $Z$-mediated new physics effects in Kaon observables in some of the models and significant effects in $B_{s, d^{-}}$observables. $\varepsilon^{\prime} / \varepsilon$ can easily be made consistent with the data, implying then uniquely the suppression of $K_{L} \rightarrow \pi^{0} \nu \bar{\nu}$. Significant enhancements of $\operatorname{Br}\left(K^{+} \rightarrow \pi^{+} \nu \bar{\nu}\right)$ are still possible. We point out that the combination of NP effects to $|\Delta F|=2$ and $|\Delta F|=1$ observables in a given meson system generally allows to determine the masses of VLQs in a given representation independently of the size of VLQ couplings.

KeYwords: Beyond Standard Model, CP violation, Heavy Quark Physics, Kaon Physics ARXIV EPRINT: 1609.04783 


\section{Contents}

1 Introduction 1

2 The VLQ models $\quad 4$

2.1 VLQ representations 4

2.2 Yukawa interactions of VLQs 5

2.2.1 $\mathrm{G}_{\mathrm{SM}} \quad 5$

$2.2 .2 \mathrm{G}_{\mathrm{SM}}^{\prime}(S) \quad 5$

2.2.3 $\mathrm{G}_{\mathrm{SM}}^{\prime}(\Phi) \quad 5$

2.2.4 Yukawa couplings of several representations 6

$\begin{array}{lll}2.3 & \text { Scalar sectors } & 7\end{array}$

$\begin{array}{lll}3 & \text { Decoupling of VLQs } & 7\end{array}$

3.1 Tree-level decoupling and $Z$ and $Z^{\prime}$ effects 9

3.1.1 $\mathrm{G}_{\mathrm{SM}}$-models 11

$\begin{array}{lll}3.1 .2 \mathrm{G}_{\mathrm{SM}^{-}}^{\prime} \text { models } & 12\end{array}$

$\begin{array}{lll}3.2 & \text { Decoupling at one-loop level } & 13\end{array}$

$\begin{array}{ll}3.3 & \text { Renormalisation group evolution } \\ \end{array}$

4 Implications for the down-quark sector $\quad 18$

$\begin{array}{ll}4.1|\Delta F|=2 & 19\end{array}$

$4.2|\Delta F|=1$ : semi-leptonic $d_{j} \rightarrow d_{i}+(\ell \bar{\ell}, \nu \bar{\nu}) \quad 21$

$4.3|\Delta F|=1$ : hadronic $d_{j} \rightarrow d_{i} q \bar{q}$ and $\varepsilon^{\prime} / \varepsilon \quad 22$

5 Patterns of flavour violation $\quad \mathbf{2 5}$

$5.1|\Delta F|=2 \quad 25$

$5.2|\Delta F|=1 \quad 27$

$\begin{array}{lll}5.3 & \text { Determination of } M & 29\end{array}$

$\begin{array}{lll}5.4 & \text { Kaon and } B \text {-meson systems } & 30\end{array}$

6 Numerics $\quad 32$

6.1 $\mathrm{G}_{\mathrm{SM}}$ models 34

$6.2 \mathrm{G}_{\mathrm{SM}}^{\prime}(\Phi)$ model $\quad 42$

$\begin{array}{lll}7 & \text { Summary and conclusions } & 45\end{array}$

A Scalar sectors of $\mathrm{G}_{\mathrm{SM}^{\prime}}^{- \text {models }} \quad 49$

A.1 $\mathrm{G}_{\mathrm{SM}}^{\prime}(S)$ models $\quad 49$

A.2 $\mathrm{G}_{\mathrm{SM}}^{\prime}(\Phi)$ models $\quad 50$ 
B VLQ decoupling and RG effects

B.1 $\psi^{2} \varphi^{2} D$ operators $\quad 51$

B.2 $\psi^{2} \varphi^{3}$ operators $\quad 52$

B.3 Top-Yukawa RG effects 53

C Master formulae for $K$ and $B$ decays

C.1 $|\Delta F|=2 \quad 55$

$\begin{array}{lll}\text { C.2 } & d_{j} \rightarrow d_{i} \nu \bar{\nu} & 56\end{array}$

C.3 $d_{j} \rightarrow d_{i} \ell \bar{\ell} \quad 57$

C.4 $d_{j} \rightarrow d_{i} q \bar{q}$ and $\varepsilon^{\prime} / \varepsilon \quad 59$

$\begin{array}{ll}\text { D Statistical approach and numerical input } & 60\end{array}$

\section{Introduction}

Among the simplest renormalisable extensions of the Standard Model (SM) that do not introduce any additional fine tunings of parameters are models in which the only new particles are vector-like fermions. Such fermions can be much heavier than the SM ones as they can acquire masses in the absence of electroweak symmetry breaking. If in the process of this breaking mixing with the SM fermions occurs, the generation of flavour-changing neutral currents (FCNC) mediated by the SM $Z$ boson is a generic implication. If in addition the gauge group is extended by a second $\mathrm{U}(1)$ factor, a new heavy gauge boson $Z^{\prime}$ is present and additional heavy scalars are necessary to provide mass for the $Z^{\prime}$ and to break the extended gauge-symmetry group down to the SM gauge group. There is a rich literature on FCNCs implied by the presence of vector-like quarks (VLQs), see in particular [1-12].

The goal of the present paper is an extensive study of patterns of flavour violation in models with VLQs that are based on the following gauge groups:

$$
\begin{aligned}
\mathrm{G}_{\mathrm{SM}} & \equiv \mathrm{SU}(3)_{\mathrm{c}} \otimes \mathrm{SU}(2)_{\mathrm{L}} \otimes \mathrm{U}(1)_{\mathrm{Y}}, \\
\mathrm{G}_{\mathrm{SM}}^{\prime} & \equiv \mathrm{G}_{\mathrm{SM}} \otimes \mathrm{U}(1)_{\mathrm{L}_{\mu}-\mathrm{L}_{\tau}} .
\end{aligned}
$$

The choice of the particular symmetry group $\mathrm{U}(1)_{\mathrm{L}_{\mu}-\mathrm{L}_{\tau}}[13,14]$ is phenomenologically motivated by the fact that it allows in a simple manner to address successfully the LHCb anomalies $[9,15]$, while being anomaly-free and containing less parameters than general $Z^{\prime}$ models [16].

In our paper we will be guided by the analyses in refs. $[3,11,17]$ which identified all renormalisable models with additional fermions residing in a single vector-like complex representation of the SM gauge group with a mass $M$. It turns out that there are 11 models where new fermions have the proper quantum numbers so that they can couple in a renormalisable manner to the SM Higgs and SM fermions, thereby implying new sources of flavour violation. Our analysis will concentrate on FCNCs in the $K, B_{d}$ and $B_{s}$ systems, 
therefore only the five models with couplings to down quarks are relevant for us, as specified in section 2. We call this class of models $\mathrm{G}_{\mathrm{SM}}$-models.

Consequently the models based on the gauge group $\mathrm{G}_{\mathrm{SM}}^{\prime}$ are called $\mathrm{G}_{\mathrm{SM}}^{\prime}$-models. The VLQs in these models belong to the same representations under $\mathrm{G}_{\mathrm{SM}}$ as in $\mathrm{G}_{\mathrm{SM}}$-models, but are additionally charged under $\mathrm{U}(1)_{\mathrm{L}_{\mu}-\mathrm{L}_{\tau}}$. These models also contain new heavy scalars.

As we will discuss in detail in section 2 and section 5 , the patterns of flavour violation in $\mathrm{G}_{\mathrm{SM}}$-models and $\mathrm{G}_{\mathrm{SM}}^{\prime}$-models differ significantly from each other:

- In $\mathrm{G}_{\mathrm{SM}}$-models Yukawa interactions of the SM scalar doublet $H$ involving ordinary quarks and VLQs imply flavour-violating $Z$ couplings to ordinary quarks, which then dominate $|\Delta F|=1 \mathrm{FCNC}$ transitions. However, the situation in $|\Delta F|=2$ transitions is much more involved and depends on whether right-handed $(\mathrm{RH})$ or left-handed (LH) flavour-violating quark couplings to the $Z$ are present. If they are $\mathrm{RH}$ the effects of renormalisation group (RG) evolution from $M$ (the common VLQ mass) down to the electroweak scale, $\mu_{\mathrm{EW}}$, generate left-right operators [18] via top-Yukawa induced mixing. These operators are strongly enhanced through QCD $\mathrm{RG}$ effects below the electroweak scale and in the case of the $K$ system through chirally enhanced hadronic matrix elements. They dominate then new physics (NP) contributions to $\varepsilon_{K}$, but in the $B_{s, d}$ meson systems for VLQ-masses above $5 \mathrm{TeV}$ they have to compete with contributions from box diagrams with VLQs [11]. If they are LH the Yukawa enhancement is less important, because left-right operators are not present and box diagrams play an important role both in the $B_{s, d}$ and $K$ systems.

- In $\mathrm{G}_{\mathrm{SM}^{\prime}}^{\prime}$-models the pattern of flavour violation depends on the scalar sector involved. We consider only models in which at least one of the additional scalars is charged under $\mathrm{U}(1)_{\mathrm{L}_{\mu}-\mathrm{L}_{\tau}}$ in such a way that Yukawa couplings between the given VLQ and ordinary quarks are allowed. If this is the case for a new scalar which is just a singlet $S$ under the SM group, the latter imply flavour-violating $Z^{\prime}$ couplings to ordinary quarks without any FCNCs mediated by the $Z$. In the following we refer to these models as $\mathrm{G}_{\mathrm{SM}}^{\prime}(S)$-models. If, on the other hand, such a Yukawa coupling requires the scalar to be a doublet $\Phi$, both tree-level $Z^{\prime}$ and $Z$ contributions to flavour observables will be present. Their relative size depends on the model parameters, specifically the $Z^{\prime}$ mass. In these cases we introduce again an additional scalar singlet, but without Yukawa couplings, since otherwise the $Z^{\prime}$ mass would have to be of the order of the electroweak scale, which is phenomenologically very difficult to achieve. In the following we refer to these models as $\mathrm{G}_{\mathrm{SM}}^{\prime}(\Phi)$-models.

In this manner we will consider three classes of VLQ models with rather different patterns of flavour violation:

$$
\mathrm{G}_{\mathrm{SM}}, \quad \mathrm{G}_{\mathrm{SM}}^{\prime}(S), \quad \mathrm{G}_{\mathrm{SM}}^{\prime}(\Phi),
$$

in which $|\Delta F|=1$ FCNCs are mediated by the $Z, Z^{\prime}$ and both, respectively. In $\mathrm{G}_{\mathrm{SM}}^{\prime}(\Phi)$ models $|\Delta F|=2$ transitions are dominated for $M \geq 5 \mathrm{TeV}$ by box diagrams with VLQs and scalar exchanges, while in the $\mathrm{G}_{\mathrm{SM}}^{\prime}(S)$ models also tree-level $Z^{\prime}$ exchanges can play 
an important, sometimes dominant, role. A particular feature of $\mathrm{G}_{\mathrm{SM}}$ models are the topYukawa induced RG effects to $|\Delta F|=2$ transitions that are largest for $\mathrm{RH}$ scenarios and are absent in $\mathrm{G}_{\mathrm{SM}}^{\prime}$ models.

In [11] an extensive analysis of the $\mathrm{G}_{\mathrm{SM}}$-models has been performed and a subset of $\mathrm{G}_{\mathrm{SM}^{-}}^{\prime}$-models has been analyzed in $[9,15]$. Therefore it is mandatory for us to state what is new in our article regarding these models:

- The authors of [11] concentrated on the derivation of bounds on the Yukawa couplings as functions of $M$ but did not study the correlations between various flavour observables which is the prime target of our paper. Similar comments apply to [9].

- NP contributions to flavour observables depend in each model on the products of complex Yukawa couplings $\lambda_{s}^{*} \lambda_{d}, \lambda_{b}^{*} \lambda_{d}$ and $\lambda_{b}^{*} \lambda_{s}$ for $s \rightarrow d, b \rightarrow d$ and $b \rightarrow s$ transitions, respectively, as well as the VLQ mass $M$. This structure allows to set one of the $\lambda_{q}$-phases to zero, such that each model depends on only five Yukawa parameters and $M$, implying a number of correlations between flavour observables. The strongest correlations are, however, still found between observables corresponding to the same flavour-changing transition, and we concentrate our analysis on them. The correlations between observables with different transitions are weaker, but could turn out to be useful in the future when the data and theory improve, in particular in the context of models for Yukawa couplings.

- An important novelty of our paper, relative to $[9,11,15]$, is the inclusion of the ratio $\varepsilon^{\prime} / \varepsilon$ in our study. Recent analyses indicate that the measurement of $\varepsilon^{\prime} / \varepsilon$ is significantly above its SM prediction [19-22]; it is hence of interest to see which of the models analyzed by us, if any, are capable of addressing this tension and what the consequences for other observables are.

- Another important novelty in the context of VLQ models and $|\Delta F|=2$ transitions in general is the inclusion of the effects of RG top-Yukawa evolution from $M$ to the electroweak scale that turn out to be very important for models with RH currents through the generation of left-right operators contributing to these transitions as mentioned above. This changes markedly the pattern of flavour violation in such models relative to models with LH currents where no left-right operators are generated.

Our paper is organized as follows. In section 2 we present the particle content of the considered VLQ models, together with the gauge interactions, Yukawa interactions and the scalar sector. In section 3 we perform the decoupling of the VLQs and construct the effective field theory $\left(\mathrm{G}_{\mathrm{SM}}^{(\prime)}\right.$-EFT) for each model for scales $\mu_{\mathrm{EW}}<\mu<M$. Section 4 is devoted to the matching of these EFTs to phenomenological ones describing $|\Delta F|=1,2$ processes below the scale $\mu_{\mathrm{EW}}$. This results in explicit flavour-violating couplings of the $Z$ and $Z^{\prime}$ to the SM quarks. These enter the effective Lagrangians for the various flavour-changing processes, from which we derive the explicit formulae for the considered observables. In section 5 we describe the patterns of flavour violation expected in different models, summarizing them with the help of two DNA tables. In section 6, after formulating our strategy for 
the phenomenology, we present numerical results of our study. We conclude in section 7 . Several appendices collect additional information on the models, the decoupling of VLQs, $\mathrm{RG}$ equations in the $\mathrm{G}_{\mathrm{SM}}-\mathrm{EFT}$, the considered decays, some technical details and the input and statistical procedure used in the numerical analysis.

\section{The VLQ models}

Throughout the article we focus on models with vector-like fermions residing in complex representations, either of the the $\mathrm{SM}$ gauge group $\mathrm{G}_{\mathrm{SM}}$ or its extension by an additional gauged $\left(L_{\mu}-L_{\tau}\right)$ symmetry, $\mathrm{U}(1)_{\mathrm{L}_{\mu}-\mathrm{L}_{\tau}}$. For both models we adapt the usual SM fermion content of the three generations $(i=1,2,3)$ of quarks $\left(q_{L}^{i}=\left(u_{L}^{i}, d_{L}^{i}\right)^{T}, u_{R}^{i}, d_{R}^{i}\right)$ and leptons $\left(L_{L}^{i}=\left(\nu_{i}, \ell_{L}^{i}\right)^{T}, \ell_{R}^{i}\right)$, which acquire masses via spontaneous symmetry breaking from the standard scalar $\mathrm{SU}(2)_{\mathrm{L}}$ doublet $H$.

The gauged $\left(L_{\mu}-L_{\tau}\right)$ symmetry is anomaly-free in the SM [13, 14]. The only nonvanishing $\left(L_{\mu}-L_{\tau}\right)$ charges of the SM fermions are introduced as

$$
Q^{\prime}\left(L_{L}^{2}\right)=Q^{\prime}\left(\mu_{R}\right)=Q_{\ell}^{\prime}, \quad Q^{\prime}\left(L_{L}^{3}\right)=Q^{\prime}\left(\tau_{R}\right)=-Q_{\ell}^{\prime} .
$$

Here $L_{L}^{2}=\left(\nu_{\mu}, \mu_{L}\right)$ and $L_{L}^{3}=\left(\nu_{\tau}, \tau_{L}\right)$ are left-handed $\mathrm{SU}(2)_{\mathrm{L}}$ doublets and $\mu_{R}$ and $\tau_{R}$ right-handed singlets. We normalize the $\left(L_{\mu}-L_{\tau}\right)$ charges of the leptons without loss of generality by setting $Q_{\ell}^{\prime}=1$. The SM quarks do not couple directly to the $\mathrm{U}(1)_{\mathrm{L}_{\mu}-\mathrm{L}_{\tau}}$ gauge boson $Z^{\prime}$. However, such couplings are generated in $\mathrm{G}_{\mathrm{SM}}^{\prime}$ models through Yukawa interactions of SM quarks with VLQs that couple directly to $Z^{\prime}$.

\subsection{VLQ representations}

As we are mainly interested in the phenomenology of down-quark physics, we will restrict our analysis to $\mathrm{SU}(3)_{\mathrm{c}}$ triplets and consider the following five models with $\mathrm{SU}(2)_{\mathrm{L}}$ singlets, doublets and triplets:

$$
\begin{array}{rrrr}
\text { singlets : } & D(1,-1 / 3,-X), & (\mathrm{V}) \\
\text { doublets : } & Q_{V}(2,+1 / 6,+X), & Q_{d}(2,-5 / 6,-X), & (\mathrm{IX}, \mathrm{XI}) \\
\text { triplets : } & T_{d}(3,-1 / 3,-X), & T_{u}(3,+2 / 3,+X), & (\mathrm{VII}, \mathrm{VIII})
\end{array}
$$

where the transformation properties are indicated as $\left(\mathrm{SU}(2)_{\mathrm{L}}, \mathrm{U}(1)_{\mathrm{Y}}, \mathrm{U}(1)_{\mathrm{L}_{\mu}-\mathrm{L}_{\tau}}\right)$, i.e. $X$ denotes the charge under $\mathrm{U}(1)_{\mathrm{L}_{\mu}-\mathrm{L}_{\tau}}$. It is implied that in $\mathrm{G}_{\mathrm{SM}^{-}}$-models the $\mathrm{U}(1)_{\mathrm{L}_{\mu}-\mathrm{L}_{\tau}}$ charge should be omitted. The representations $D, Q_{V}, Q_{d}, T_{d}, T_{u}$ correspond to the models V, IX, XI, VII, VIII introduced in ref. [11], where a complete list of renormalisable models with vector-like fermions under $\mathrm{G}_{\mathrm{SM}}$ can be found, see also [3, 17]. Concerning $\mathrm{G}_{\mathrm{SM}}^{\prime}$, the combination of representations $D, Q_{V}$ and additionally $\mathrm{U}(1,+2 / 3,-X)$ has been studied first in [9].

The kinetic and gauge interactions of the new VLQs are given by

$$
\mathcal{L}_{\text {kin }}=\bar{D}\left(i \not \mathcal{D}-M_{D}\right) D+\sum_{a=V, d} \bar{Q}_{a}\left(i \not \mathcal{D}-M_{Q_{a}}\right) Q_{a}+\sum_{a=d, u} \operatorname{Tr}\left[\bar{T}_{a}\left(i \not \mathcal{D}-M_{T_{a}}\right) T_{a}\right]
$$


with appropriate covariant derivatives $\mathcal{D}_{\mu}$ and we follow [11] for the triplet representations as given in (2.13) and (2.14) of that paper. The masses $M$ of the VLQs introduce a new scale, which we will assume to be significantly larger than all other scales. The covariant derivative is, omitting the $\mathrm{SU}(3)_{\mathrm{c}}$ part,

$$
\mathcal{D}_{\mu}=\partial_{\mu}-i g_{1} \frac{\sigma^{a}}{2} W_{\mu}^{a}-i g_{2} Y B_{\mu}-i g^{\prime} Q^{\prime} \hat{Z}_{\mu}^{\prime}
$$

with the gauge couplings $g_{2,1}$ and $g^{\prime}$ of $\mathrm{SU}(2)_{\mathrm{L}}, \mathrm{U}(1)_{\mathrm{Y}}$ and $\mathrm{U}(1)_{\mathrm{L}_{\mu}-\mathrm{L}_{\tau}}$, respectively, and charges $Y$ and $Q^{\prime}$ of $\mathrm{U}(1)_{\mathrm{Y}}$ and $\mathrm{U}(1)_{\mathrm{L}_{\mu}-\mathrm{L}_{\tau}}$. The Pauli-matrices are denoted by $\sigma^{a}$. The "hat" on $\hat{Z}_{\mu}^{\prime}$ indicates that we deal here with the gauge eigenstate and not mass eigenstate, see (A.7).

\subsection{Yukawa interactions of VLQs}

\subsection{1 $\mathrm{G}_{\mathrm{SM}}$}

The scalar sector consists of the SM scalar doublet $H$ with its usual scalar potential. The VLQs interact with SM quarks $\left(q_{L}, u_{R}, d_{R}\right)$ via Yukawa interactions

$$
\begin{aligned}
-\mathcal{L}_{\mathrm{Yuk}}(H)= & \left(\lambda_{i}^{D} H^{\dagger} \bar{D}_{R}+\lambda_{i}^{T_{d}} H^{\dagger} \bar{T}_{d R}+\lambda_{i}^{T_{u}} \widetilde{H}^{\dagger} \bar{T}_{u R}\right) q_{L}^{i} \\
& +\lambda_{i}^{V_{u}} \bar{u}_{R}^{i} \widetilde{H}^{\dagger} Q_{V L}+\bar{d}_{R}^{i}\left(\lambda_{i}^{V_{d}} H^{\dagger} Q_{V L}+\lambda_{i}^{Q_{d}} \widetilde{H}^{\dagger} Q_{d L}\right)+\text { h.c. },
\end{aligned}
$$

where $\widetilde{H} \equiv i \sigma_{2} H^{*}$. The complex-valued Yukawa couplings $\lambda_{i}^{\mathrm{VLQ}}$ give rise to mixing with the SM quarks and flavour-changing $Z$-couplings, which have been worked out in detail $[3,11]$ and are discussed in section 3.1.

\subsection{2 $\mathrm{G}_{\mathrm{SM}}^{\prime}(S)$}

In models with an additional $\mathrm{U}(1)_{\mathrm{L}_{\mu}-\mathrm{L}_{\tau}}$ the scalar sector has to be extended in order to generate the mass of the corresponding gauge boson $Z^{\prime}$. A complex scalar $S(1,0, X)$ $\left(\mathrm{SU}(3)_{\mathrm{c}}\right.$ singlet) is added in the minimal version. As VLQs are charged under $\mathrm{U}(1)_{\mathrm{L}_{\mu}-\mathrm{L}_{\tau}}$, their Yukawa couplings with the SM doublet $H$ are forbidden, but the ones involving $S$ are allowed for $Q_{S}^{\prime}= \pm Q_{\mathrm{VLQ}}^{\prime}$ and given by [9]

$$
-\mathcal{L}_{\text {Yuk }}(S)=\left(\lambda_{i}^{D} \bar{d}_{R}^{i} D_{L}+\lambda_{i}^{V} \bar{Q}_{V R} q_{L}^{i}\right) S+\text { h.c. . }
$$

In fact this scalar system is sufficient for models with VLQs having $\mathrm{U}(1)_{\mathrm{Y}}$ charges $Y=-1 / 3$ and $+1 / 6$ of the SM fermions $d_{R}$ and $q_{L}$, respectively. In the following we refer to these models as $\mathrm{G}_{\mathrm{SM}}^{\prime}(S)$-models. The special feature of these models is that because of the absence of tree-level $Z$ contributions tree-level $Z^{\prime}$ exchanges dominate $\Delta F=1$ transitions and in some part of the parameter space can also compete with contributions from box diagrams with VLQs and scalars in the case of $\Delta F=2$ transitions.

\subsection{3 $\mathrm{G}_{\mathrm{SM}}^{\prime}(\Phi)$}

For VLQs with $\mathrm{G}_{\mathrm{SM}}$ quantum numbers different from one of the SM quark fields, the simple extension by a scalar singlet is not possible. In a next-to-minimal version we therefore add 
to the scalar sector an additional scalar $\mathrm{SU}(2)_{\mathrm{L}}$ doublet $\Phi(2,+1 / 2, X)$, besides the SMlike $H(2,+1 / 2,0)$. We require $|X| \neq 1,2$ in order to avoid lepton-flavour violating (LFV) Yukawa couplings — see for example [23] — and in consequence there are no LFV $Z^{\prime}$ couplings, which are subject to strong constraints at low energies. The vacuum expectation value (VEV) of $\Phi$ gives an unavoidable contribution to the $Z^{\prime}$ mass of the order of the electroweak scale, contributes to the mass of $H$ and generates potentially large $Z-Z^{\prime}$ mass mixing effects. The latter would be strongly constrained by electroweak precision tests [24], in particular there would be sizeable corrections to the $Z$ couplings to muons. In order to avoid these difficulties, $\Phi$ is accompanied by an additional complex scalar singlet $S(1,0, Y)$, which breaks the $\mathrm{U}(1)_{\mathrm{L}_{\mu}-\mathrm{L}_{\tau}}$ symmetry at the TeV scale. The $L_{\mu}-L_{\tau}$ charge of $S$ is chosen to be $Y=X / 2$ in order to avoid the appearance of a Goldstone boson in the scalar sector and to forbid Yukawa couplings of $S$ with SM fermions and VLQs.

The Yukawa interactions of the VLQs with $\Phi$ are

$$
-\mathcal{L}_{\text {Yuk }}(\Phi)=\left(\lambda_{i}^{D} \Phi^{\dagger} \bar{D}_{R}+\lambda_{i}^{T_{d}} \Phi^{\dagger} \bar{T}_{d R}+\lambda_{i}^{T_{u}} \widetilde{\Phi}^{\dagger} \bar{T}_{u R}\right) q_{L}^{i}+\lambda_{i}^{Q_{d}} \widetilde{\Phi}^{\dagger} \bar{d}_{R}^{i} Q_{d L}+\text { h.c. },
$$

with $\widetilde{\Phi} \equiv i \sigma_{2} \Phi^{*}$ and we will refer to these models as $\mathrm{G}_{\mathrm{SM}}^{\prime}(\Phi)$-models. We note that the structure of couplings equals the one of $\mathrm{G}_{\mathrm{SM}}$ models given in eq. (2.5) upon $H \leftrightarrow \Phi$. For the VLQ $D(1,-1 / 3, X)$ we consider thus two versions, one in $\mathrm{G}_{\mathrm{SM}}^{\prime}(S)$ and one in the $\mathrm{G}_{\mathrm{SM}}^{\prime}(\Phi)$-model. We refrain from the same procedure for $Q_{V}(2,+1 / 6, X)$. In $\mathrm{G}_{\mathrm{SM}}^{\prime}(\Phi)$ models FCNCs are mediated by both $Z$ and $Z^{\prime}$ but in the case of $\Delta F=2$ transitions box diagrams with VLQs and scalars play the dominant role for sufficiently large $M$.

For ease of notation, we will sometimes refrain below from explicitly labelling the $\lambda_{i}$ by the VLQ representation, as should be done if several of them are considered simultaneously.

\subsubsection{Yukawa couplings of several representations}

In our numerics we will consider one VLQ representation at a time as this simplifies the analysis significantly. In particular the number of parameters is quite limited. Still it is useful to make a few comments on the structure of flavour-violating interactions and at various places in our paper to state how our formulae would be modified through the presence of several VLQ representations in a given model. We plan to return to the phenomenology of such models in the future.

When admitting several VLQ representations $F^{m}$ and $F^{n}$ simultaneously, potentially additional locally gauge-invariant Yukawa couplings $\sim \widetilde{\lambda}_{m n} \bar{F}_{L}^{m} \varphi_{m n} F_{R}^{n}$ with $\varphi_{m n}=H$ have to be included in the case of $\mathrm{G}_{\mathrm{SM}}$-models [3]. They give rise to flavour-changing neutral Higgs currents at tree level. In the $\mathrm{G}_{\mathrm{SM}^{-}}^{\prime}$-models the $\mathrm{U}(1)_{\mathrm{L}_{\mu}-\mathrm{L}_{\tau}}$-charges of the additional $\varphi_{m n}=S, \Phi$ have been chosen following the criteria explained above, which fixes in turn the $\mathrm{U}(1)_{\mathrm{L}_{\mu}-\mathrm{L}_{\tau}}$-charges of the VLQs. In consequence such couplings to $\varphi_{m n}=S, \Phi$ are not permitted, however they are still allowed for $\varphi_{m n}=H$, which has zero $\mathrm{U}(1)_{\mathrm{L}_{\mu}-\mathrm{L}_{\tau}}$-charge. In $\mathrm{G}_{\mathrm{SM}}^{\prime}(S)$ models, only the particular choice of the $\mathrm{U}(1)_{\mathrm{L}_{\mu}-\mathrm{L}_{\tau}}$ charges $Q_{Q_{V}}^{\prime}=-Q_{D}^{\prime}[9]$ forbids these couplings to $H$, whereas the choice $Q_{Q_{V}}^{\prime}=Q_{D}^{\prime}$ would allow them, due to the possibility to replace $\bar{Q}_{V R} q_{L}^{i} \rightarrow \bar{q}_{L}^{i} Q_{V R}$ in eq. (2.6), which maintains gauge invariance since $S$ is a singlet. On the other hand, in $\mathrm{G}_{\mathrm{SM}}^{\prime}(\Phi)$ models such couplings arise for $Q_{d}$ with $D$ and $T_{d}$. 
Another important consequence of the presence of several representations is the generation of left-right $|\Delta F|=2$ operators in models with both LH and RH currents via box diagrams discussed in section 3.2, which is the case when singlets or triplets together with doublets are present. In the case of a single representation such operators can also be generated in models with doublets through the top-Yukawa RG evolution from $M$ to the electroweak scale, see section 3.3.

\subsection{Scalar sectors}

In the $\mathrm{G}_{\mathrm{SM}}$-models, the scalar sector contains only the standard doublet $H(2,+1 / 2,0)$, which provides masses to gauge bosons and standard fermions in the course of spontaneous symmetry breaking of $\mathrm{SU}(2)_{\mathrm{L}} \otimes \mathrm{U}(1)_{\mathrm{Y}} \rightarrow \mathrm{U}(1)_{\mathrm{em}}$ via the $\mathrm{VEV} v \simeq 246 \mathrm{GeV}$, where

$$
\langle H\rangle=(0, v / \sqrt{2})^{T} .
$$

In $\mathrm{G}_{\mathrm{SM}}^{\prime}(S)$-models the doublet $H(2,+1 / 2,0)$ fulfils again the same role, whereas the singlet $S(1,0, X)$ provides via its $\operatorname{VEV}\langle S\rangle=v_{S} / \sqrt{2}$ a mass for the additional $\mathrm{U}(1)_{\mathrm{L}_{\mu}-\mathrm{L}_{\tau}}$ $Z^{\prime}$-gauge boson

$$
M_{Z^{\prime}}^{2}=g^{\prime 2} v_{S}^{2} X^{2}
$$

In $\mathrm{G}_{\mathrm{SM}}^{\prime}(\Phi)$-models the doublet $\Phi_{2} \equiv H(2,+1 / 2,0)$ gives masses to the chiral fermions, whereas $\Phi_{1} \equiv \Phi(2,+1 / 2, X)$ contributes to the masses of the $Z$ and $Z^{\prime}$ gauge bosons in combination with $S(1,0, X / 2) .{ }^{1}$ The neutral components of the doublets acquire VEV's

$$
\left\langle\Phi_{a}^{0}\right\rangle=\frac{v_{a}}{\sqrt{2}}, \quad \tan \beta \equiv \frac{v_{2}}{v_{1}}, \quad v=\sqrt{v_{1}^{2}+v_{2}^{2}} \simeq 246 \mathrm{GeV},
$$

with $0 \leq \beta \leq \pi / 2$. In this case, neutral gauge boson mixing occurs with details given in appendix A.2.

Further details on the scalar sectors of the $\mathrm{G}_{\mathrm{SM}}^{\prime}(S)$ and $\mathrm{G}_{\mathrm{SM}}^{\prime}(\Phi)$ models are collected in appendix A.1 and A.2, respectively. In table 1 we summarize all $\mathrm{G}_{\mathrm{SM}^{\prime}}$-models and indicate which diagrams dominate NP contributions to $|\Delta F|=1$ and $|\Delta F|=2$ transitions in a given model.

\section{Decoupling of VLQs}

The VLQ models are characterised by the masses $M$ of the VLQs, the various Yukawa couplings $\lambda_{i}^{\mathrm{VLQ}}(i=1,2,3)$ of section 2.2 and the VEVs of the respective scalar sectors, see section 2.3. The present lower bound on $M$ from the LHC is in the ballpark of $1 \mathrm{TeV}$, while the lower bounds on $M_{Z^{\prime}}$ are typically close to $3 \mathrm{TeV}$ if $Z^{\prime}$ has a direct coupling to light quarks. But as emphasized in $[9,15,25], Z^{\prime}$ of $\mathrm{U}(1)_{\mathrm{L}_{\mu}-\mathrm{L}_{\tau}}$ does not have such couplings, implying a much weaker lower bound on its mass, which could in fact be as low

\footnotetext{
${ }^{1}$ This convention corresponds to that of the Type I 2HDM.
} 


\begin{tabular}{|r|c|c|c|c|}
\hline VLQ Representation & Scalar Singlet & Scalar Doublets & $|\Delta F|=1$ & $|\Delta F|=2$ \\
\hline$D_{a}(3,1,-1 / 3,-X)$ & $S(1,1,0, X)$ & $H(1,2,1 / 2,0)$ & $Z^{\prime}$ & $Z^{\prime}$, Box \\
$D_{b}(3,1,-1 / 3,-X)$ & $S(1,1,0, X / 2)$ & $\Phi_{1}(1,2,1 / 2, X), \Phi_{2}(1,2,1 / 2,0)$ & $Z^{\prime}, Z$ & Box \\
$Q_{V}(3,2,+1 / 6,+X)$ & $S(1,1,0, X)$ & $H(1,2,1 / 2,0)$ & $Z^{\prime}$ & $Z^{\prime}$, Box \\
$Q_{d}(3,2,-5 / 6,-X)$ & $S(1,1,0, X / 2)$ & $\Phi_{1}(1,2,1 / 2, X), \Phi_{2}(1,2,1 / 2,0)$ & $Z^{\prime}, Z$ & Box \\
$T_{d}(3,3,-1 / 3,-X)$ & $S(1,1,0, X / 2)$ & $\Phi_{1}(1,2,1 / 2, X), \Phi_{2}(1,2,1 / 2,0)$ & $Z^{\prime}, Z$ & Box \\
$T_{u}(3,3,+2 / 3,+X)$ & $S(1,1,0, X / 2)$ & $\Phi_{1}(1,2,1 / 2, X), \Phi_{2}(1,2,1 / 2,0)$ & $Z^{\prime}, Z$ & Box \\
\hline
\end{tabular}

Table 1. Fermion and scalar representations under $\mathrm{SU}(3)_{\mathrm{c}} \otimes \mathrm{SU}(2)_{\mathrm{L}} \otimes \mathrm{U}(1)_{\mathrm{Y}} \otimes \mathrm{U}(1)_{\mathrm{L}_{\mu}-\mathrm{L}_{\tau}}$ in $\mathrm{G}_{\mathrm{SM}^{-}}^{\prime}$ models. In the last two columns we show which diagrams dominate NP contributions to $|\Delta F|=1$ and $|\Delta F|=2$ transitions for $M \geq 5 \mathrm{TeV}$.

as the electroweak scale and even lower. While it could also be as heavy as the VLQ mass, we will assume the hierarchy

$$
M_{Z} \lesssim M_{Z^{\prime}} \ll M, \quad \text { or equivalently } \quad v \lesssim v_{S} \ll M
$$

in order to simplify the analysis. It is then natural to decouple first the VLQs and to consider EFTs for $\mathrm{G}_{\mathrm{SM}}$ and $\mathrm{G}_{\mathrm{SM}}^{\prime}$ valid between the scales $\mu_{M} \sim M$ and $\mu_{\mathrm{EW}} \sim v \simeq v_{S}$. These are subsequently matched in one step onto $\mathrm{SU}(3)_{\mathrm{c}} \otimes \mathrm{U}(1)_{\mathrm{em}}$-invariant phenomenological EFTs of $|\Delta F|=1,2$ decays, which are valid between $\mu_{\mathrm{EW}}$ and $\mu_{b} \sim m_{b}$, where $m_{b}$ denotes the bottom mass. The coefficients determined in the process will indicate which operators are the most important. In principle one could consider an intermediate EFT which is constructed by integrating out $Z^{\prime}$ and the new scalars before integrating out top quark, $W$ and $Z$, but from the point of view of renormalisation group effects, integrating out all these heavy fields simultaneously appears to be an adequate approximation.

In this section we present the results from the decoupling of the VLQs that are important for our phenomenological applications within the framework of the $\mathrm{G}_{\mathrm{SM}}^{(\prime)}$-EFTs. The matching step of the $\mathrm{G}_{\mathrm{SM}^{\prime}}^{\left({ }^{\prime}\right.}$-EFTs to phenomenological EFT's of $|\Delta F|=1,2$ processes at the scale $\mu_{\mathrm{EW}}$ is given in section 4 . The Lagrangian of the $\mathrm{G}_{\mathrm{SM}}^{(\prime)}$-EFT consists of the dimension-four interactions of the light fields and dimension six interactions generated by the decoupling of VLQs

$$
\mathcal{L}_{\mathrm{G}_{\mathrm{SM}}^{(\prime)}-\mathrm{EFT}}=\mathcal{L}_{\mathrm{dim}-4}+\sum_{a} \mathcal{C}_{a} \mathcal{O}_{a}
$$

which are invariant under either $\mathrm{G}_{\mathrm{SM}}$ or $\mathrm{G}_{\mathrm{SM}}^{\prime}$, depending on the model. Thus in $\mathrm{G}_{\mathrm{SM}^{-}}$ models $\mathcal{L}_{\operatorname{dim}-4}$ coincides with the SM Lagrangian and the corresponding non-redundant set of operators of dimension six has been classified in ref. [26]. In $\mathrm{G}_{\mathrm{SM}^{\prime}}^{\prime}$-models operators that are invariant under $\mathrm{G}_{\mathrm{SM}}^{\prime}$ must be added, which involve the $Z^{\prime}$-boson and the additional scalar singlets and/or doublets. The Wilson coefficients $\mathcal{C}_{a}{ }^{2}$ are effective couplings, which are suppressed by $1 / M^{2}$ and their effects on observables by $v_{i}^{2} / M^{2}$ compared to the SM,

\footnotetext{
${ }^{2}$ The Wilson coefficients of $\mathrm{G}_{\mathrm{SM}}^{(\prime)}$-EFTs are denoted with calligraphic $\mathcal{C}_{i}$, whereas the ones of phenomenological EFTs with $C_{i}$.
} 
with $v_{i}=\left(v, v_{1}, v_{S}\right)$ depending on the model. They are determined at the scale $\mu_{M}$ when decoupling VLQs. The decoupling proceeds either by explicit matching calculations starting at tree-level and including subsequently higher orders or by integrating them out in the path integral method [3]. The tree-level decoupling has been known for a long time for $\mathrm{G}_{\mathrm{SM}}$ models [3] and is given for $\mathrm{G}_{\mathrm{SM}}^{\prime}(S)$ models in ref. [9].

Within the EFT, RG equations allow to evolve the Wilson coefficients from $\mu_{M}$ down to $\mu_{\mathrm{EW}}$. In leading logarithmic approximation and retaining only the first logarithm (1stLLA) it has the approximate solution

$$
\mathcal{C}_{a}\left(\mu_{\mathrm{EW}}\right)=\left[\delta_{a b}-\frac{\gamma_{a b}}{(4 \pi)^{2}} \ln \frac{\mu_{M}}{\mu_{\mathrm{EW}}}\right] \mathcal{C}_{b}\left(\mu_{M}\right),
$$

which holds as long as the second term remains small compared to the first. The anomalous dimension matrices (ADM) $\gamma_{a b}$ depend in general on couplings of the gauge, Yukawa and scalar sectors and are known for the $\mathrm{G}_{\mathrm{SM}}-\mathrm{EFT}$ [27-29]. Largest contributions might be expected for the case of $\gamma_{a b} \propto Y_{u}^{\dagger} Y_{u} \sim y_{t}^{2}$ mixing due to the top-quark Yukawa coupling $y_{t} \sim 1$ of the order of a few percent in the case of self-mixing $(a=b)$ and from the mixing due to QCD under $\alpha_{s}$. On the other hand, for $a \neq b$ non-zero Wilson coefficients can be generated at 1stLLA order. ${ }^{3}$ In particular, as we will see below, in the case of models with right-handed neutral currents left-right operators can be generated in this manner with profound direct impact on $|\Delta F|=2$ transitions, thereby affecting the predictions for $|\Delta F|=1$ observables.

The VLQs have a very limited set of couplings to light fields, which are either via gauge interactions (2.3) to the gauge bosons or via Yukawa interactions (2.5)-(2.7) to light w.r.t. to VLQ mass $M-\mathrm{SM}$ quarks and scalars $\varphi=H, S$ or $\Phi$, depending on the model. At tree-level, this particular structure of interactions can give rise only to flavour-changing $Z$ and $Z^{\prime}$ couplings, whereas all other decoupling effects are loop-suppressed [30].

The decoupling of the VLQs proceeds in the unbroken phase of $\mathrm{SU}(2)_{\mathrm{L}} \otimes \mathrm{U}(1)_{\mathrm{Y}}$, hence quark fields are flavour-eigenstates and neutral components of scalar fields are without VEV at this stage. After the RG evolution from $\mu_{M}$ to $\mu_{\mathrm{EW}}$, spontaneous symmetry breaking will take place within the $\mathrm{G}_{\mathrm{SM}}^{(\prime)}$-EFTs and the transformation from flavour- to mass-eigenstates for fermions and gauge bosons can be performed, accounting for the dimension six part in eq. (3.2).

\subsection{Tree-level decoupling and $Z$ and $Z^{\prime}$ effects}

The couplings of the VLQs permit at tree level only a dimension six contribution from the generic 4-point diagram in figure 1a. Since its dimension-five contribution vanishes [3], it is equivalent to consider the 5-point diagram figure $1 \mathrm{~b}$, where either $\mathrm{SU}(2)_{\mathrm{L}}$ or $\mathrm{U}(1)_{\mathrm{Y}}$ gauge bosons in $\mathrm{G}_{\mathrm{SM}}$-models or in addition a $\hat{Z}^{\prime}$ in $\mathrm{G}_{\mathrm{SM}^{\prime}}^{\prime}$-models is radiated off the VLQ [3, 9]. As a consequence, in $\mathrm{G}_{\mathrm{SM}^{-}}$and $\mathrm{G}_{\mathrm{SM}^{-}}^{\prime}$ models only operators of the type $\psi^{2} \varphi^{2} D \propto$

\footnotetext{
${ }^{3}$ Note that the 1stLLA neglects "secondary mixing" effects that are present in LLA, i.e. summing all large logarithms, because although operator $\mathcal{O}_{A}$ might not have ADM entry with operator $\mathcal{O}_{B}$ (no "direct mixing"), it can still contribute to the Wilson coefficient $C_{B}\left(\mu_{\mathrm{EW}}\right)$, if it mixes directly with some operator $\mathcal{O}_{C}$ that in turn mixes directly into $\mathcal{O}_{B}$.
} 


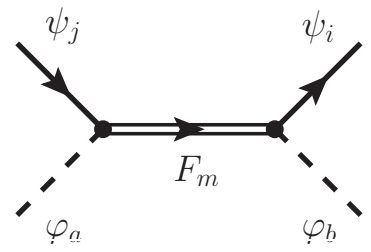

(a)

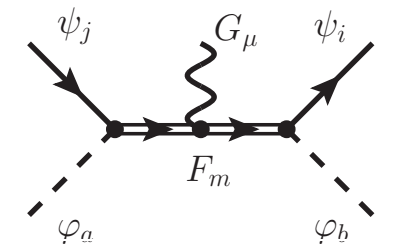

(b)

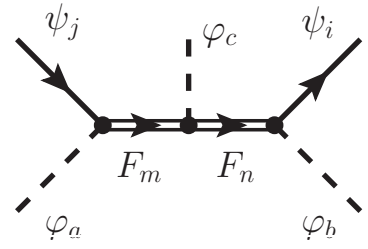

(c)

Figure 1. Tree-level graphs (a) and (b) of the decoupling of a VLQ $F_{m}$ that give rise to $\psi^{2} \varphi^{2} D$ operators. They proceed via their Yukawa interactions with scalars $\varphi=(H, S, \Phi)$ and SM quarks $\psi=\left(q_{L}, u_{R}, d_{R}\right)$. The gauge boson $G_{\mu}$ depends on the representation. Tree-level graph (c) requires two representations $F_{m, n}$ with a Yukawa coupling via $\varphi_{c}$ and give rise to $\psi^{2} \varphi^{3}$ operators.

\begin{tabular}{|c|c|c|c|c|c|}
\hline & $\mathrm{G}_{\mathrm{SM}}$ & & $\mathrm{G}_{\mathrm{SM}}^{\prime}(S)$ & & $\mathrm{G}_{\mathrm{SM}}^{\prime}(\Phi)$ \\
\hline \multicolumn{6}{|c|}{$\psi^{2} \varphi^{2} D$} \\
\hline $\mathcal{O}_{H q}^{(1)}$ & $\left(H^{\dagger} i \overleftrightarrow{\mathcal{D}_{\mu}} H\right)\left[\bar{q}_{L}^{i} \gamma^{\mu} q_{L}^{j}\right]$ & $\mathcal{O}_{S q}$ & $\left(S^{*} i \overleftrightarrow{\mathcal{D}_{\mu}} S\right)\left[\bar{q}_{L}^{i} \gamma^{\mu} q_{L}^{j}\right]$ & $\mathcal{O}_{\Phi q}^{(1)}$ & $\left(\Phi^{\dagger} i \overleftrightarrow{\mathcal{D}_{\mu}} \Phi\right)\left[\bar{q}_{L}^{i} \gamma^{\mu} q_{L}^{j}\right]$ \\
\hline $\mathcal{O}_{H q}^{(3)}$ & $\left(H^{\dagger} i \overleftrightarrow{\mathcal{D}}_{\mu}^{a} H\right)\left[\bar{q}_{L}^{i} \sigma^{a} \gamma^{\mu} q_{L}^{j}\right]$ & - & - & $\mathcal{O}_{\Phi q}^{(3)}$ & $\left(\Phi^{\dagger} i \overleftrightarrow{\mathcal{D}}_{\mu}^{a} \Phi\right)\left[\bar{q}_{L}^{i} \sigma^{a} \gamma^{\mu} q_{L}^{j}\right]$ \\
\hline $\mathcal{O}_{H u}$ & $\left(H^{\dagger} i \overleftrightarrow{\mathcal{D}_{\mu}} H\right)\left[\bar{u}_{R}^{i} \gamma^{\mu} u_{R}^{j}\right]$ & $\mathcal{O}_{S u}$ & $\left(S^{*} i \overleftrightarrow{\mathcal{D}}_{\mu} S\right)\left[\bar{u}_{R}^{i} \gamma^{\mu} u_{R}^{j}\right]$ & $\mathcal{O}_{\Phi u}$ & $\left(\Phi^{\dagger} i \overleftrightarrow{\mathcal{D}_{\mu}} \Phi\right)\left[\bar{u}_{R}^{i} \gamma^{\mu} u_{R}^{j}\right]$ \\
\hline $\mathcal{O}_{H d}$ & $\left(H^{\dagger} i \overleftrightarrow{\mathcal{D}_{\mu}} H\right)\left[\bar{d}_{R}^{i} \gamma^{\mu} d_{R}^{j}\right]$ & $\mathcal{O}_{S d}$ & $\left(S^{*} i \overleftrightarrow{\mathcal{D}_{\mu}} S\right)\left[\bar{d}_{R}^{i} \gamma^{\mu} d_{R}^{j}\right]$ & $\mathcal{O}_{\Phi d}$ & $\left(\Phi^{\dagger} i{\overleftrightarrow{\mathcal{D}_{\mu}}} \Phi\right)\left[\bar{d}_{R}^{i} \gamma^{\mu} d_{R}^{j}\right]$ \\
\hline $\mathcal{O}_{\text {Hud }}$ & $\left(\widetilde{H}^{\dagger} i \mathcal{D}_{\mu} H\right)\left[\bar{u}_{R}^{i} \gamma^{\mu} d_{R}^{j}\right]$ & - & - & $\mathcal{O}_{\Phi u d}$ & $\left(\widetilde{\Phi}^{\dagger} i \mathcal{D}_{\mu} \Phi\right)\left[\bar{u}_{R}^{i} \gamma^{\mu} d_{R}^{j}\right]$ \\
\hline \multicolumn{6}{|c|}{$\psi^{2} \varphi^{3}$} \\
\hline $\mathcal{O}_{u H}$ & $\left(H^{\dagger} H\right)\left[\bar{q}_{L}^{i} u_{R}^{j} \widetilde{H}\right]$ & $\mathcal{O}_{u S}$ & $\left(S^{*} S\right)\left[\bar{q}_{L}^{i} u_{R}^{j} \widetilde{H}\right]$ & $\mathcal{O}_{u \Phi}$ & $\left(\Phi^{\dagger} \Phi\right)\left[\bar{q}_{L}^{i} u_{R}^{j} \widetilde{H}\right]$ \\
\hline $\mathcal{O}_{d H}$ & $\left(H^{\dagger} H\right)\left[\bar{q}_{L}^{i} d_{R}^{j} H\right]$ & $\mathcal{O}_{d S}$ & $\left(S^{*} S\right)\left[\bar{q}_{L}^{i} d_{R}^{j} H\right]$ & $\mathcal{O}_{d \Phi}$ & $\left(\Phi^{\dagger} \Phi\right)\left[\bar{q}_{L}^{i} d_{R}^{j} H\right]$ \\
\hline
\end{tabular}

Table 2. We follow the definitions of [26] for $\psi^{2} \varphi^{2} D$ operators, except for the signs of gauge couplings in the covariant derivatives, and $\left(\psi^{2} \varphi^{3}+\right.$ h.c.) operators in the case of $\mathrm{G}_{\mathrm{SM}}$ models and extend them to $\mathrm{G}_{\mathrm{SM}}^{\prime}(S)$ and $\mathrm{G}_{\mathrm{SM}}^{\prime}(\Phi)$-models $(\varphi=H, S, \Phi)$. Superindices $i, j=1,2,3$ on quark fields denote the generations. These are all operators that could arise from tree-level decoupling of VLQs, depending on the model.

$\left(\varphi^{\dagger} i \overleftrightarrow{\mathcal{D}}_{\mu} \varphi\right)\left[\bar{\psi}_{i} \gamma^{\mu} \psi_{j}\right](\varphi=H, S, \Phi)$ receive non-vanishing contributions at tree-level, which are projected in part onto $\psi^{2} \varphi^{3}$-type operators via equation of motions (EOM) [26, 31]. We list the corresponding definitions of the operators in table 2, following the notation of [26] in the case of the $\mathrm{G}_{\mathrm{SM}}$-EFT and extending it to $\mathrm{G}_{\mathrm{SM}^{\prime}}$-EFTs.

After spontaneous symmetry breaking the $\psi^{2} \varphi^{3}$ operators contribute to the quark masses $m_{\psi}(\psi=u, d)$ at the scale $\mu_{\mathrm{EW}}$ via

$$
m_{\psi}^{i j}=\frac{v_{2}}{\sqrt{2}}\left(Y_{\psi}^{i j}-\frac{v_{2}^{2}}{2} \mathcal{C}_{\psi H}^{i j}-\frac{v_{S}^{2}}{2} \mathcal{C}_{\psi S}^{i j}-\frac{v_{1}^{2}}{2} \mathcal{C}_{\psi \Phi}^{i j}\right),
$$

which allows to substitute Yukawa couplings $Y_{\psi}$ in terms of measured $m_{\psi}$ and new physics parameters $\mathcal{C}_{\psi^{2} \varphi^{3}} \propto Y_{\psi} \mathcal{C}_{\psi^{2} \varphi^{2} D}$, see appendix B.2. If several representations of VLQs are 
present in a given model and two of them $F_{m, n}$ couple to a scalar $\varphi_{c}{ }^{4}$ via Yukawa couplings $\widetilde{\lambda}_{m n}$, a third possibility is allowed at tree-level depicted in figure 1c, which contributes directly to $\psi^{2} \varphi^{3}$ operators and gives rise to flavour-changing neutral $H \bar{\psi}_{i} \psi_{j}$ interactions at tree-level [3]. The various possibilities for $\mathrm{G}_{\mathrm{SM}}$ models, where $\varphi_{c}=H$, can be found in [3].

The relation of quark masses to the Yukawa interactions (3.4) includes now also $1 / M^{2}$ contributions. Their diagonalisation proceeds as usual for the quark fields with the help of $3 \times 3$ unitary rotations in flavour space:

$$
\psi_{L} \rightarrow V_{L}^{\psi} \psi_{L}, \quad \psi_{R} \rightarrow V_{R}^{\psi} \psi_{R}
$$

implying

$$
V_{L}^{\psi \dagger} m_{\psi} V_{R}^{\psi}=m_{\psi}^{\text {diag }}, \quad \quad V=\left(V_{L}^{u}\right)^{\dagger} V_{L}^{d},
$$

with diagonal up- and down-quark masses $m_{\psi}^{\text {diag }}$ and the unitary quark-mixing matrix $V$. In the limit of vanishing dimension-six contributions, $V$ will become the Cabibbo-KobayashiMaskawa (CKM) matrix of the SM. Throughout we will assume for down quarks the weak basis in which the mass term $m_{d}$ is already diagonal, implying $q_{L}=\left(V^{\dagger} u_{L}, d_{L}\right)^{T}$. This fixes also the definition of the Wilson coefficients $\mathcal{C}_{\psi^{2} \varphi^{2} D}$ (for more details see [32]) and the basis for the VLQ Yukawa couplings $\lambda_{i}^{\mathrm{VLQ}}$.

After spontaneous symmetry breaking the $\psi^{2} \varphi^{2} D$ operators give rise to flavourchanging $Z$ and $Z^{\prime}$ interactions for fermions $(f=\ell, u, d)$, which we parametrise as follows:

$$
\begin{aligned}
\mathcal{L}_{\mathrm{VLQ}}^{(Z)} & =\bar{f}^{i}\left[\Delta_{L}^{i j}(Z) \gamma^{\mu} P_{L}+\Delta_{R}^{i j}(Z) \gamma^{\mu} P_{R}\right] f^{j} Z_{\mu}, \\
\mathcal{L}^{\left(Z^{\prime}\right)} & =\bar{f}^{i}\left[\Delta_{L}^{i j}\left(Z^{\prime}\right) \gamma^{\mu} P_{L}+\Delta_{R}^{i j}\left(Z^{\prime}\right) \gamma^{\mu} P_{R}\right] f^{j} Z_{\mu}^{\prime} .
\end{aligned}
$$

For completeness, we provide the matching conditions for the Wilson coefficients in appendix B. We note that RG effects have been neglected in (3.7) and (3.8) since they are only due to self-mixing of $\psi^{2} \varphi^{2} D$ operators as listed in appendix B.3.

The flavour-diagonal $(i=j)$ couplings of leptons to the $Z$ will be set to the ones of the $\mathrm{SM}$ as corrections from NP to them are in $\mathrm{G}_{\mathrm{SM}}$-models one-loop suppressed. This is also the case of $\mathrm{G}_{\mathrm{SM}}^{\prime}(S)$ models where $Z$ does not play any role in FCNCs. In $\mathrm{G}_{\mathrm{SM}}^{\prime}(\Phi)$ models modifications of the $Z f \bar{f}$ couplings come from $Z-Z^{\prime}$ mixing. These shifts are relevant for leptons in partial widths of $Z \rightarrow \ell \bar{\ell}$ (see appendix A.2) and could be of relevance in electroweak precision tests. In the semi-leptonic $|\Delta F|=1$ FCNCs we will include them for consistency in $\mathrm{G}_{\mathrm{SM}}^{\prime}(\Phi)$ models, although they are negligible in comparison to other effects.

\subsubsection{G $\quad$ SM-models $_{\text {SM }}$}

In the case of $\mathrm{G}_{\mathrm{SM}}$-models, the decoupling of VLQs gives the results for $\Delta_{L, R}(Z)$ couplings collected for down-quarks in table 3 , where

$$
\Delta^{i j} \equiv \frac{\lambda_{i}^{*} \lambda_{j}}{g_{Z}} \frac{M_{Z}^{2}}{M^{2}}, \quad g_{Z} \equiv \sqrt{g_{1}^{2}+g_{2}^{2}} .
$$

Except for the sign in the case of $T_{u}$, our results agree with those in [11]. Furthermore, also non-zero couplings to up-type quarks arise [11] but they will not play any role in our paper.

\footnotetext{
${ }^{4}$ As discussed above $\varphi_{c}=H$ in $\mathrm{G}_{\mathrm{SM}}$ and $\mathrm{G}_{\mathrm{SM}}^{\prime}$-models.
} 


\begin{tabular}{|c|c|c|c|c|c|c|}
\hline Coupling & $q$ & $D$ & $Q_{V}$ & $Q_{d}$ & $T_{d}$ & $T_{u}$ \\
\hline \multirow{2}{*}{$\Delta_{L}^{q_{i} q_{j}}(Z)$} & $d$ & $\Delta^{i j}$ & 0 & 0 & $\Delta^{i j} / 2$ & $-\Delta^{i j}$ \\
& $u$ & 0 & 0 & 0 & $V_{i m} \Delta^{m n}\left(V^{\dagger}\right)_{n j}$ & $-V_{i m} \Delta^{m n}\left(V^{\dagger}\right)_{n j} / 2$ \\
\hline \multirow{2}{*}{$\Delta_{R}^{q_{i} q_{j}}(Z)$} & $d$ & 0 & $-\left(\Delta^{i j}\right)^{*}$ & $\left(\Delta^{i j}\right)^{*}$ & 0 & 0 \\
& $u$ & 0 & $\left(\Delta_{u}^{i j}\right)^{*}$ & 0 & 0 & 0 \\
\hline
\end{tabular}

Table 3. $\Delta_{L, R}^{q_{i} q_{j}}(Z)$ for down- and up-type-quark couplings $(i, j=1,2,3)$ to the $Z$ boson in $\mathrm{G}_{\mathrm{SM}^{-}}$ models. Here $V_{i j}$ is the CKM matrix and $\Delta_{u}=\Delta\left(\lambda_{i}^{V_{d}} \rightarrow \lambda_{i}^{V_{u}}\right)$, see (2.5).

\subsection{2 $\mathrm{G}_{\mathrm{SM}}^{\prime}$-models}

In the $\mathrm{G}_{\mathrm{SM}}^{\prime}$-models, the $\left(L_{\mu}-L_{\tau}\right)$ symmetry fixes the $Z^{\prime}$ coupling to leptons to be

$$
\Delta_{L}^{\ell \bar{\ell}}\left(Z^{\prime}\right)=\Delta_{R}^{\ell \bar{\ell}}\left(Z^{\prime}\right)=\Delta_{L}^{\nu_{\ell} \bar{\nu}_{\ell}}\left(Z^{\prime}\right)=g^{\prime} Q_{\ell}^{\prime},
$$

with $Q_{\ell}^{\prime}=\{0,+1,-1\}$ for $\ell=\{e, \mu, \tau\}$. Here we have neglected $Z-Z^{\prime}$ mixing effects existing in $\mathrm{G}_{\mathrm{SM}}^{\prime}(\Phi)$-models. However, for consistency we have to include these effects in the couplings of the $Z$ to leptons

$$
\Delta_{L}^{\ell \bar{\ell}}(Z)=-g_{Z}\left(\frac{1}{2}-s_{W}^{2}\right)+g^{\prime} Q_{\ell}^{\prime} \xi_{Z Z^{\prime}}, \quad \Delta_{R}^{\ell \bar{\ell}}(Z)=g_{Z} s_{W}^{2}+g^{\prime} Q_{\ell}^{\prime} \xi_{Z Z^{\prime}},
$$

to first order in the small mixing angle $\xi_{Z Z^{\prime}}$ (see appendix A.2 for details). On the other hand, the gauge couplings to quarks are model dependent.

In $\mathrm{G}_{\mathrm{SM}}^{\prime}(S)$-models the scalar sector of $S$ and $H$ generates only non-zero quark couplings to $Z^{\prime}$, whereas in $\mathrm{G}_{\mathrm{SM}}^{\prime}(\Phi)$-models the scalar sector of $S, H$ and $\Phi$ gives rise to non-zero couplings of SM quarks to both $Z^{\prime}$ and $Z$. We define

$$
G^{i j} \equiv-\frac{\lambda_{i}^{*} \lambda_{j}}{2 X g^{\prime}} \frac{M_{Z^{\prime}}^{2}}{M^{2}}, \quad \quad K^{i j} \equiv c_{\beta}^{2} \frac{\lambda_{i}^{*} \lambda_{j}}{g_{Z}} \frac{M_{Z}^{2}}{M^{2}}=c_{\beta}^{2} \Delta^{i j},
$$

with $\Delta^{i j}$ defined in eq. (3.9) and the $Z-Z^{\prime}$ mixing angle [see (A.9)]

$$
\xi_{Z Z^{\prime}} \simeq r^{\prime} c_{\beta}^{2} \frac{M_{Z}^{2}}{M_{Z^{\prime}}^{2}}, \quad \quad r^{\prime} \equiv \frac{2 X g^{\prime}}{g_{Z}} .
$$

Here $c_{\beta} \equiv \cos \beta$ is a parameter associated with the scalar sector (see $(2.10)$ ) of $\mathrm{G}_{\mathrm{SM}}^{\prime}(\Phi)$ models, i.e. $v_{1}=v \cos \beta$. The $\xi_{Z Z^{\prime}}$ describes $Z-Z^{\prime}$ mixing, which is phenomenologically constrained to be small, $\xi_{Z Z^{\prime}}<0.1$, due to constraints from the $Z$-boson mass, $M_{Z}$, and partial widths $Z \rightarrow \ell \bar{\ell}$ measured at LEP, as described in more detail in appendix A.2. The down- and up-quark couplings to $Z^{\prime}$ and $Z$ are collected for these models in table 4 . We confirm previous findings [9] for the $\mathrm{G}_{\mathrm{SM}}^{\prime}(S)$-models.

We note that the $Z^{\prime}$ couplings are suppressed/enhanced by the ratio $r^{\prime}$ w.r.t. the $Z$ couplings. Enhancement takes place for $2 g^{\prime} X>g_{Z} \approx 0.75$, such that for example $r^{\prime} \approx 3$ can be reached with $g^{\prime} X \approx 1.1$, still within the perturbative regime. The couplings of $T_{d}$ and $T_{u}$ differ just by a sign and factors $1 / 2$. In distinction to $Z$-contributions in $\mathrm{G}_{\mathrm{SM}^{-}}$ models, both $Z$ - and $Z^{\prime}$-contributions in $\mathrm{G}_{\mathrm{SM}}^{\prime}(\Phi)$ models decouple with large $\tan \beta$, see $K^{i j}$ in eq. (3.12). 


\begin{tabular}{|c|c|c|c|c|c|}
\hline Model & $q$ & $\Delta_{L}^{q_{i} q_{j}}\left(Z^{\prime}\right)$ & $\Delta_{R}^{q_{i} q_{j}}\left(Z^{\prime}\right)$ & $\Delta_{L}^{q_{i} q_{j}}(Z)$ & $\Delta_{R}^{q_{i} q_{j}}(Z)$ \\
\hline \multicolumn{6}{|c|}{$\mathrm{G}_{\mathrm{SM}}^{\prime}(S)$} \\
\hline \multirow{3}{*}{$\begin{array}{c}D \\
Q_{V}\end{array}$} & $d$ & 0 & $\left(G^{i j}\right)^{*}$ & 0 & 0 \\
\hline & $d$ & $G^{i j}$ & 0 & 0 & 0 \\
\hline & $u$ & $V_{i m} G^{m n}\left(V^{\dagger}\right)_{n j}$ & 0 & 0 & 0 \\
\hline \multicolumn{6}{|c|}{$\mathrm{G}_{\mathrm{SM}}^{\prime}(\Phi)$} \\
\hline$D$ & $d$ & $-r^{\prime} K^{i j}$ & 0 & {$\left[1-r^{\prime} \xi_{Z Z^{\prime}}\right] K^{i j}$} & 0 \\
\hline$Q_{d}$ & $d$ & 0 & $-r^{\prime}\left(K^{i j}\right)^{*}$ & 0 & {$\left[1-r^{\prime} \xi_{Z Z^{\prime}}\right]\left(K^{i j}\right)^{*}$} \\
\hline \multirow{2}{*}{$T_{d}$} & $d$ & $-r^{\prime} K^{i j} / 2$ & 0 & {$\left[1-r^{\prime} \xi_{Z Z^{\prime}}\right] K^{i j} / 2$} & 0 \\
\hline & $u$ & $-r^{\prime} V_{i m} K^{m n}\left(V^{\dagger}\right)_{n j}$ & 0 & {$\left[1-r^{\prime} \xi_{Z Z^{\prime}}\right] V_{i m} K^{m n}\left(V^{\dagger}\right)_{n j}$} & 0 \\
\hline \multirow{2}{*}{$T_{u}$} & $d$ & $r^{\prime} K^{i j}$ & 0 & $-\left[1-r^{\prime} \xi_{Z Z^{\prime}}\right] K^{i j}$ & 0 \\
\hline & $u$ & $r^{\prime} V_{i m} K^{m n}\left(V^{\dagger}\right)_{n j} / 2$ & 0 & $-\left[1-r^{\prime} \xi_{Z Z^{\prime}}\right] V_{i m} K^{m n}\left(V^{\dagger}\right)_{n j} / 2$ & 0 \\
\hline
\end{tabular}

Table 4. $\Delta_{L, R}^{q_{i} q_{j}}\left(Z^{\prime}\right)$ and $\Delta_{L, R}^{q_{i} q_{j}}(Z)$ for down- and up-type quark couplings $(i, j=1,2,3)$ in $\mathrm{G}_{\mathrm{SM}^{-}}^{\prime}$ models. Here $V_{i j}$ is the CKM matrix.

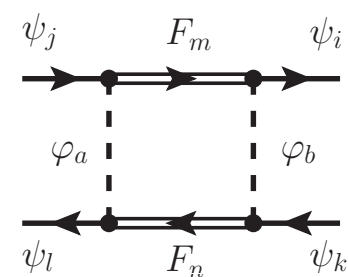

(a)

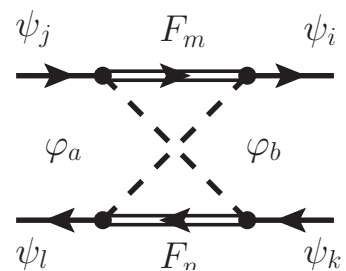

(b)

Figure 2. Box graphs for the decoupling of VLQs in representations $F_{m, n}$ due to their Yukawa interactions with scalars $\varphi=H, S, \Phi$ and SM quarks $\psi=\left(q_{L}, d_{R}, u_{R}\right)$. The crossed graph appears for certain representations $F_{m} \neq F_{n}$. The $|\Delta F|=2$ graphs are found for $k=j$ and $l=i$.

\subsection{Decoupling at one-loop level}

All other decoupling processes proceed via loops. Those that would lead to non-canonical kinetic terms in the $\mathrm{G}_{\mathrm{SM}}^{(\prime)}$-EFTs can be absorbed by a suitable choice of wave-function renormalisation constants in the full theory above the scale $\mu_{M}$, resulting in non-minimal renormalisation of interactions and giving rise to finite threshold effects of coupling constants. In $\mathrm{G}_{\mathrm{SM}}^{\prime}$-models this is the case for kinetic mixing of $B_{\mu}$ and $\hat{Z}_{\mu}^{\prime}$, which enters our analysis only as a higher order effect.

All other effects enter as dimension six operators. The ones with four quarks are most important for quark-flavour phenomenology. They involve only VLQ-Yukawa interactions, as depicted in figure $2 \mathrm{a}$ and figure $2 \mathrm{~b}$, and give rise to $\psi^{4}$-type operators, among which are also $|\Delta F|=2$ operators. Here we match directly to the operators present in the phenomenological EFT of $|\Delta F|=2$ decays, using the conventions in appendix C.1, avoiding 
thereby the intermediate matching to the $\mathrm{G}_{\mathrm{SM}}$-invariant form. ${ }^{5}$ Still, we outline this step for completeness here. In the VLQ models considered, there are four relevant $\psi^{4}$ operators in $\mathrm{G}_{\mathrm{SM}}^{(\prime)}$-EFTs at the VLQ scale $\mu_{M}$ and a fifth operator is generated due to QCD mixing via $\mathrm{RG}$ evolution from $\mu_{M}$ to $\mu_{\mathrm{EW}}$. These are the $(\bar{L} L)(\bar{L} L)$ operators

$$
\left[\mathcal{O}_{q q}^{(1)}\right]_{i j k l}=\left[\bar{q}_{L}^{i} \gamma_{\mu} q_{L}^{j}\right]\left[\bar{q}_{L}^{k} \gamma^{\mu} q_{L}^{l}\right], \quad\left[\mathcal{O}_{q q}^{(3)}\right]_{i j k l}=\left[\bar{q}_{L}^{i} \gamma_{\mu} \sigma^{a} q_{L}^{j}\right]\left[\bar{q}_{L}^{k} \gamma^{\mu} \sigma^{a} q_{L}^{l}\right]
$$

the $(\bar{L} L)(\bar{R} R)$ operators

$$
\left[\mathcal{O}_{q d}^{(1)}\right]_{i j k l}=\left[\bar{q}_{L}^{i} \gamma_{\mu} q_{L}^{j}\right]\left[\bar{d}_{R}^{k} \gamma^{\mu} d_{R}^{l}\right], \quad\left[\mathcal{O}_{q d}^{(8)}\right]_{i j k l}=\left[\bar{q}_{L}^{i} \gamma_{\mu} T^{A} q_{L}^{j}\right]\left[\bar{d}_{R}^{k} \gamma^{\mu} T^{A} d_{R}^{l}\right],
$$

and the $(\bar{R} R)(\bar{R} R)$ operator

$$
\left[\mathcal{O}_{d d}\right]_{i j k l}=\left[\bar{d}_{R}^{i} \gamma_{\mu} d_{R}^{j}\right]\left[\bar{d}_{R}^{k} \gamma^{\mu} d_{R}^{l}\right]
$$

with $k l=i j$ for $|\Delta F|=2$ processes and the $T^{A}$ denoting $\mathrm{SU}(3)_{\mathrm{c}}$ colour generators. Their Wilson coefficients are matched to the ones of the $|\Delta F|=2$ phenomenological EFT at the electroweak scale $\mu_{\mathrm{EW}}[32]$ as

$$
\begin{array}{ll}
C_{\mathrm{VLL}}^{i j}=-\mathcal{N}_{i j}^{-1}\left(\left[\mathcal{C}_{q q}^{(1)}\right]_{i j i j}+\left[\mathcal{C}_{q q}^{(3)}\right]_{i j i j}\right), & C_{\mathrm{VRR}}^{i j}=-\mathcal{N}_{i j}^{-1}\left[\mathcal{C}_{d d}\right]_{i j i j} \\
C_{\mathrm{LR}, 1}^{i j}=-\mathcal{N}_{i j}^{-1}\left(\left[\mathcal{C}_{q d}^{(1)}\right]_{i j i j}-\frac{\left[\mathcal{C}_{q d}^{(8)}\right]_{i j i j}}{2 N_{c}}\right), & C_{\mathrm{LR}, 2}^{i j}=\mathcal{N}_{i j}^{-1}\left[\mathcal{C}_{q d}^{(8)}\right]_{i j i j}
\end{array}
$$

where $\mathcal{N}_{i j}$ is given in (C.2). Here we anticipate this matching to the VLQ scale $\mu_{M}$ as there are no RG effects of phenomenological importance for the discussion of $B$-meson and Kaon sectors. For more details see section 3.3, where also QCD mixing is given for these operators. Since the Wilson coefficients of these operators are generated at $\mu_{M}$ at one-loop, their interplay with other sectors in quark-flavour physics due to RG mixing are considered higher order and hence beyond the scope of our work.

In $\mathrm{G}_{\mathrm{SM}}$-models VLQs contribute to $|\Delta F|=2$ operators $O_{a}^{i j}$ for $a=\mathrm{VLL}, \mathrm{VRR}, \mathrm{LR} 1$ via box diagrams (see figures $2 \mathrm{a}$ and $2 \mathrm{~b}$ ), which contain two heavy VLQ propagators with representations $F_{m}$ and $F_{n}$ and massless components of the standard doublet $H=$ $\left(H^{+}, H^{0}\right)^{T}$. These box diagrams yield the general structure of the Wilson coefficients

$$
\mathcal{C}_{a}^{i j}\left(\mu_{M}\right)=\frac{\eta_{m n}}{(4 \pi)^{2}} \frac{\Lambda_{i j}^{m} \Lambda_{i j}^{n}}{\mathcal{N}_{i j}} f_{1}\left(M_{m}, M_{n}\right)
$$

at the scale $\mu_{M}$. Here the prefactor corresponds to the SM normalisation of the $|\Delta F|=2$ EFT, see (C.2). The function

$$
f_{1}\left(M_{m}, M_{n}\right)=\frac{\ln \left(M_{m}^{2} / M_{n}^{2}\right)}{M_{m}^{2}-M_{n}^{2}}, \quad \text { with } \quad f_{1}\left(M_{m}, M_{m}\right)=\frac{1}{M_{m}^{2}},
$$

\footnotetext{
${ }^{5}$ Note that the set of $\psi^{4}$-type operators is the same in all $\mathrm{G}_{\mathrm{SM}}^{(\prime)}$ models and a non-redundant set can be found in ref. [26].
} 


\begin{tabular}{|c|c|c|c|c|c|}
\hline$\left(F_{m}, F_{n}\right)$ & $D$ & $Q_{d}$ & $Q_{V}$ & $T_{d}$ & $T_{u}$ \\
\hline$D$ & $\mathrm{VLL},+1 / 8$ & $\mathrm{LR} 1,+1 / 4$ & $\mathrm{LR} 1,-1 / 4$ & $\mathrm{VLL},+1 / 16$ & $\mathrm{VLL},-1 / 8$ \\
$Q_{d}$ & & $\mathrm{VRR},+1 / 4$ & $\mathrm{VRR},-1 / 4$ & $\mathrm{LR} 1,+3 / 8$ & $\mathrm{LR} 1,-3 / 8$ \\
$Q_{V}$ & & & $\mathrm{VRR},+1 / 4$ & $\mathrm{LR} 1,-3 / 8$ & $\mathrm{LR} 1,+3 / 8$ \\
$T_{d}$ & & & & $\mathrm{VLL},+5 / 32$ & $\mathrm{VLL},-1 / 8$ \\
$T_{u}$ & & & & & $\mathrm{VLL},+5 / 32$ \\
\hline
\end{tabular}

Table 5. The index $a=$ VLL, VRR, LR1 appearing in eq. (3.18) for representations $\left(F_{m}, F_{n}\right)$, followed by corresponding $\eta_{m n}$.

depends on the VLQ masses of representations $F_{m, n}$. The couplings $\Lambda_{i j}^{m}$ are

$$
\begin{array}{lll}
\Lambda_{i j}^{m}=\left(\lambda_{i}^{m}\right)^{*} \lambda_{j}^{m} & \text { for } & F_{m}=D, T_{d}, T_{u}, \\
\Lambda_{i j}^{m}=\lambda_{i}^{m}\left(\lambda_{j}^{m}\right)^{*} & \text { for } & F_{m}=Q_{d}, Q_{V} .
\end{array}
$$

The index $a$ of the operator and the numerical factors $\eta_{m n}$ are collected in table 5 . Note that $a=\mathrm{VLL}$ for $F_{m, n}=D, T_{d}, T_{u}$, and $a=\mathrm{VRR}$ for $F_{m, n}=Q_{d}, Q_{V}$, whereas $a=\mathrm{LR} 1$ for $F_{m}=D, T_{d}, T_{u}$ and $F_{n}=Q_{d}, Q_{V}$. The factors $\eta_{m n}$ are positive except for interference of $F_{m}=D, Q_{d}, T_{d}$ with $F_{n}=Q_{V}, T_{u}$, because in this case the scalar propagators are crossed, which gives rise to an additional sign w.r.t. the diagram with non-crossed scalar propagators. For $F_{m}=F_{n}$, these results agree with [11] for $D, T_{u}, T_{d}$, but for $Q_{d}$ (model XI) we find an additional factor of 2 . Concerning $Q_{V}$ (model IX) we find a contribution to $\sim O_{\mathrm{VRR}}$ instead of $\sim O_{\mathrm{VLL}}$ and also opposite sign. For completeness we provide also the results for $F_{m} \neq F_{n}$.

In $\mathrm{G}_{\mathrm{SM}}^{\prime}(S)$ models we consider only VLQs $D$ and $Q_{V}$ and their interference

$$
\begin{aligned}
D: \quad \mathcal{C}_{\mathrm{VRR}} & =\frac{1}{(4 \pi)^{2}} \frac{\left(\lambda_{i}^{D} \lambda_{j}^{D *}\right)^{2}}{\mathcal{N}_{i j}} \frac{1}{8 M_{D}^{2}}, \\
Q_{V}: \quad \mathcal{C}_{\mathrm{VLL}} & =\frac{1}{(4 \pi)^{2}} \frac{\left(\lambda_{i}^{V *} \lambda_{j}^{V}\right)^{2}}{\mathcal{N}_{i j}} \frac{1}{8 M_{V}^{2}}, \\
D \times Q_{V}: \quad \mathcal{C}_{\mathrm{LR} 1} & =-\frac{1}{(4 \pi)^{2}} \frac{\left(\lambda_{i}^{D} \lambda_{j}^{D *}\right)\left(\lambda_{i}^{V *} \lambda_{j}^{V}\right)}{\mathcal{N}_{i j}} \frac{f_{1}\left(M_{D}, M_{V}\right)}{4}
\end{aligned}
$$

which agrees with [9] except for a minus sign from crossed scalar propagators in the interference term $D \times Q_{V}$.

The results for $\mathrm{G}_{\mathrm{SM}}^{\prime}(\Phi)$ models can be found straight-forwardly from the ones of the $\mathrm{G}_{\mathrm{SM}}$ models, bearing in mind that (2.5) and (2.7) are equivalent up to the replacement $H \rightarrow \Phi$. 


\subsection{Renormalisation group evolution}

The VLQ tree-level exchange in the considered VLQ scenarios generates only $\psi^{2} \varphi^{2} D$ - and $\psi^{2} \varphi^{3}$-type operators at the scale $\mu_{M}$ with nonvanishing Wilson coefficients (see appendix B)

$$
\begin{array}{rrr}
\mathrm{G}_{\mathrm{SM}}: & \mathcal{C}_{H d}, \mathcal{C}_{H q}^{(1)}, \mathcal{C}_{H q}^{(3)}, & \mathcal{C}_{u H}, \mathcal{C}_{d H}, \\
\mathrm{G}_{\mathrm{SM}}^{\prime}(S): & \mathcal{C}_{S d}, \mathcal{C}_{S q}, & \mathcal{C}_{u S}, \mathcal{C}_{d S}, \\
\mathrm{G}_{\mathrm{SM}}^{\prime}(\Phi): & \mathcal{C}_{\Phi d}, \mathcal{C}_{\Phi q}^{(1)}, \mathcal{C}_{\Phi q}^{(3)}, & \mathcal{C}_{u \Phi}, \mathcal{C}_{d \Phi},
\end{array}
$$

depending on the VLQ scenario. ${ }^{6}$ The RG evolution from $\mu_{M}$ down to $\mu_{\mathrm{EW}}$ can induce via operator mixing leading logarithmic contributions also to other classes of operators in $\mathrm{G}_{\mathrm{SM}}^{(\prime)}$ EFTs at the scale $\mu_{\mathrm{EW}}$. These operators are possibly related to a variety of processes and thus imply additional potential constraints.

The largest enhancements can appear if the ADM $\gamma_{a b}$ in (3.3) is proportional to the strong coupling $4 \pi \alpha_{s} \sim 1.4$ or the top-Yukawa coupling $y_{t} \sim 1$. Note that QCD mixing is flavour-diagonal and hence can not give rise to new genuine phenomenological effects, i.e. one can not expect qualitative changes. On the other hand, Yukawa couplings are the main source of flavour-off-diagonal interactions and we will focus on these here. The $\mathrm{SU}(2)_{\mathrm{L}}$ gauge interactions induce via ADMs $\gamma_{a b} \propto g_{2}^{2}[29]$ only intra-generational mixing between $u_{L}^{i} \leftrightarrow d_{L}^{i}$ and are parametrically smaller than $y_{t}$-induced effects, such that we do not consider them here. The $\mathrm{U}(1)_{\mathrm{Y}}$ gauge interactions are only flavour-diagonal and numerically even more suppressed.

Concerning $\mathrm{G}_{\mathrm{SM}}^{\prime}$ models, RG effects due to top-Yukawa couplings are absent for $\psi^{2} \varphi^{2} D$ and $\psi^{2} \varphi^{3}$ operators, because $\varphi=S, \Phi$ do not have Yukawa couplings to $q_{L}, u_{R}, d_{R}$, which are forbidden by their additional $\mathrm{U}(1)_{\mathrm{L}_{\mu}-\mathrm{L}_{\tau}}$ charge. Hence RG effects as discussed below are not present in these scenarios.

The ADMs due to Yukawa interactions can be found in [28] for the $\mathrm{G}_{\mathrm{SM}}$-EFT $(\varphi=H)$ and we collect the ones involving the Wilson coefficients (3.22) in appendix B.3. The RG equations of these Wilson coefficients are also coupled with those of SM couplings, such as the quartic Higgs coupling and quark-Yukawa couplings [27], but in 1stLLA they decouple. The modification of SM couplings due to dim-6 effects can be neglected when discussing the RG evolution of dim- 6 effects themselves in first approximation. Moreover, the quartic Higgs coupling is irrelevant for the processes discussed here and the quark masses are determined from low-energy experiments, i.e. much below $\mu_{\mathrm{EW}}$. Hence phenomenologically most interesting are RG effects of mixing of $\psi^{2} H^{2} D$ and $\psi^{2} H^{3}$ operators into other operator classes that do not receive tree-level matching contributions at $\mu_{M}$. Those classes are

$$
H^{6}(1), \quad H^{4} D^{2}(2), \quad \psi^{4}(5),
$$

where we list in parentheses the number of operators. ${ }^{7}$ We focus on the $\psi^{4}$ operators, which all turn out to be four-quark operators, because they are most relevant for processes

\footnotetext{
${ }^{6}$ We assume that in the VLQ scenario $Q_{V}$ the VLQ Yukawa couplings $\lambda_{i}^{V_{u}}=0$, otherwise in this scenario also $\mathcal{C}_{H u}$ and $\mathcal{C}_{H u d}$ must be considered.

${ }^{7}$ Implying footnote 6 .
} 
of down-type quarks considered here. We comment shortly on the $H^{6}$ and $H^{4} D^{2}$ classes in appendix B.3.

The RG equation (3.3) implies for a specific $a \in \psi^{4}$, see also [18],

$$
\mathcal{C}_{a}\left(\mu_{\mathrm{EW}}\right)=-\frac{1}{(4 \pi)^{2}} \ln \frac{\mu_{M}}{\mu_{\mathrm{EW}}} \sum_{b \in \psi^{2} H^{2} D} \gamma_{a b} \mathcal{C}_{b}\left(\mu_{M}\right)
$$

where $a \neq b$, such that 1stLLA contributions are one-loop suppressed w.r.t. tree-level generated $\psi^{2} H^{2} D$ contributions. Three of the $\psi^{4}$ operators $\left(\mathcal{O}_{q q}^{(1,3)}\right.$ and $\left.\mathcal{O}_{q d}^{(1)}\right)$ can mediate downtype quark $|\Delta F|=2$ processes and all five $|\Delta F|=1$ processes, see again appendix B.3.

The $|\Delta F|=1$ four-quark operators modify directly hadronic $|\Delta F|=1$ processes, whereas they enter semileptonic $|\Delta F|=1$ processes only via additional operator mixing in both SMEFT and phenomenological EFTs, therefore receiving another suppression in semileptonic processes. The 1stLLA contribution is a novel effect for $|\Delta F|=2$ processes, where it competes with the direct one-loop box contribution in VLQ models discussed in section 3.2. On the other hand, semileptonic and hadronic $|\Delta F|=1$ processes are generated directly by $\psi^{2} H^{2} D$ operators in the next matching step of $\mathrm{G}_{\mathrm{SM}}$ to phenomenological EFTs at $\mu_{\mathrm{EW}}$ (see section 4 and figure 4), which are therefore enhanced in these processes compared to the 1stLLA contributions discussed here. Consequently, the 1stLLA is oneloop suppressed in VLQ models in hadronic $|\Delta F|=1$ processes, unless the potentially novel chiral structure of the $\psi^{4}$ operators enhances a specific hadronic observable. We will return to this point in section 4.3 .

Under the transformation from weak to mass eigenstates for up-type quarks (3.5)

$$
Y_{u} \stackrel{\operatorname{dim}}{\approx} \frac{\sqrt{2}}{v} V_{L}^{u} m_{U}^{\operatorname{diag}} V_{R}^{u \dagger}=\frac{\sqrt{2}}{v} V_{\mathrm{CKM}}^{\dagger} m_{U}^{\operatorname{diag}},
$$

the corresponding ADMs of $\psi^{4}$ operators in appendix B.3 transform as

$$
\begin{aligned}
& {\left[Y_{u}^{\dagger} Y_{u}\right]_{i j}=\frac{2}{v^{2}} \sum_{k} m_{k}^{2} \delta_{k i} \delta_{k j} \approx \frac{2}{v^{2}} m_{t}^{2} \delta_{3 i} \delta_{3 j},} \\
& {\left[Y_{u} Y_{u}^{\dagger}\right]_{i j}=\frac{2}{v^{2}} \sum_{k=u, c, t} m_{k}^{2} V_{k i}^{*} V_{k j} \approx \frac{2}{v^{2}} m_{t}^{2} \lambda_{i j}^{(t)}}
\end{aligned}
$$

with up-type quark mass $m_{k}$ and the definition of CKM-products $\lambda_{i j}^{(t)}$ given in (4.1). Since the ADMs are needed here for the evolution of dim-6 Wilson coefficients themselves, we have used tree-level relations derived from the dim- 4 part of the Lagrangian only, thereby neglecting dim- 6 contributions, which would constitute a dim- 8 corrections in this context. In the sum over $k$ only the top-quark contribution is relevant $\left(m_{u, c} \ll m_{t}\right)$, if one assumes that the unitary matrix $V$ is equal to the CKM matrix up to dim- 6 corrections. ${ }^{8}$

The $|\Delta F|=2$ mediating $\psi^{4}$ operators involve the combination (3.29). We obtain via (3.26) and explicit matching conditions (B.1)

$$
\mathcal{C}_{a}^{i j}\left(\mu_{\mathrm{EW}}\right)=\frac{\kappa_{m}}{(4 \pi)^{2}} \frac{\Lambda_{i j}^{m} \lambda_{i j}^{(t)}}{\mathcal{N}_{i j}} \frac{1}{M^{2}} \frac{2 m_{t}^{2}}{v^{2}} \ln \frac{\mu_{M}}{\mu_{\mathrm{EW}}},
$$

\footnotetext{
${ }^{8}$ We expect only tiny contributions from $k=c$ in case that $i j=s d$, for $i j=b d, b s$ such contributions are entirely negligible.
} 
with $\Lambda_{i j}^{m}$ from (3.20), the chirality of the $|\Delta F|=2$ operator

$$
\begin{array}{lll}
a=\mathrm{VLL} & \text { for } & F_{m}=D, T_{d}, T_{u}, \\
a=\mathrm{LR}, 1 & \text { for } & F_{m}=Q_{d}, Q_{V},
\end{array}
$$

and the VLQ-model-dependent factor

$$
\kappa_{m}=\left(0,-\frac{1}{2},+\frac{1}{2},-\frac{1}{2},+\frac{1}{4}\right) \quad \text { for } \quad F_{m}=\left(D, Q_{d}, Q_{V}, T_{d}, T_{u}\right) .
$$

We note the relations

$$
\kappa_{m} \frac{\Lambda_{i j}^{m}}{M^{2}}=\left[\mathcal{C}_{H q}^{(1)}-\mathcal{C}_{H q}^{(3)}\right]_{i j} \quad\left(F_{m}=D, T_{d}, T_{u}\right)
$$

where the relative sign comes from relative signs in (B.23) and (B.24) when inserted in $(3.17)$ and

$$
\kappa_{m} \frac{\Lambda_{i j}^{m}}{M^{2}}=\left[\mathcal{C}_{H d}\right]_{i j}, \quad\left(F_{m}=Q_{d}, Q_{V}\right) .
$$

We point out the different flavour structure of the 1stLLA contribution (3.30) compared to the one of the direct box-contribution (3.18) discussed in the previous section section 3.2:

$$
\left.\mathcal{C}_{a}^{i j}\right|_{1 \text { stLLA }} \sim \Lambda_{i j} \times \lambda_{i j}^{(t)},\left.\quad \quad \mathcal{C}_{b}^{i j}\right|_{\text {Box }} \sim\left(\Lambda_{i j}\right)^{2},
$$

showing linear versus quadratic dependence on the product of VLQ Yukawa couplings $\Lambda_{i j}$. A detailed comparison of both contributions is given in section 5 .

The LLA RG equations of $|\Delta F|=2$ Wilson coefficients from QCD, only [33, 34], are given as

$$
\begin{aligned}
\mathcal{C}_{\mathrm{VLL}(\mathrm{VRR})}\left(\mu_{\mathrm{EW}}\right) & =\eta_{6}^{2 / 7} \mathcal{C}_{\mathrm{VLL}(\mathrm{VRR})}\left(\mu_{M}\right) \\
\mathcal{C}_{\mathrm{LR}, 1}\left(\mu_{\mathrm{EW}}\right) & =\eta_{6}^{1 / 7} \mathcal{C}_{\mathrm{LR}, 1}\left(\mu_{M}\right) \\
\mathcal{C}_{\mathrm{LR}, 2}\left(\mu_{\mathrm{EW}}\right) & =\frac{2}{3}\left(\eta_{6}^{1 / 7}-\eta_{6}^{-8 / 7}\right) \mathcal{C}_{\mathrm{LR}, 1}\left(\mu_{M}\right)+\eta_{6}^{-8 / 7} \mathcal{C}_{\mathrm{LR}, 2}\left(\mu_{M}\right)
\end{aligned}
$$

with $N_{f}=6$ denoting the number of active quark flavours and $\eta_{6}=\alpha_{s}^{(6)}\left(\mu_{M}\right) / \alpha_{s}^{(6)}\left(\mu_{\mathrm{EW}}\right)$. The initial conditions of $\mathcal{C}_{a}^{i j}\left(\mu_{M}\right)$ from box-diagrams are collected in (3.18) and (3.21). Note that $\mathcal{C}_{\mathrm{LR}, 2}\left(\mu_{M}\right)=0$, and $\mathcal{C}_{\mathrm{LR}, 1}\left(\mu_{M}\right) \neq 0$ only in the presence of several VLQ representations.

\section{Implications for the down-quark sector}

In the previous section the decoupling of the VLQs at tree-level and for $|\Delta F|=2$ at one-loop level at the scale $\mu_{M}$ has been presented, including the most important effects from the RG evolution down to the electroweak scale $\mu_{\mathrm{EW}}$. In this section we discuss the decoupling of degrees of freedom of the order of $\mu_{\mathrm{EW}}$ by matching onto phenomenological $|\Delta F|=1,2$ EFTs. In the $\mathrm{G}_{\mathrm{SM}}$-models these are the $W$ and $Z$ bosons, the top-quark and 
the standard Higgs $h^{0}$ that are all in the mass range $\mu_{\mathrm{EW}} \in[80,180] \mathrm{GeV}$. In $\mathrm{G}_{\mathrm{SM}}^{\prime}$ models the $Z^{\prime}$ and additional scalars are present, which we allow to be heavier, up to the $\sim 1 \mathrm{TeV}$ range. For the purpose of the decoupling, however, we ignore this hierarchy with the heavy standard sector $\sim 100 \mathrm{GeV}$.

In our analysis we will frequently use general formulae for flavour observables in models with tree-level neutral gauge boson exchanges that are collected in [35]. These formulae were given in terms of the so-called master one-loop functions which have been already used before in many concrete extensions of the SM, see [36] for a review. Therefore our task is to calculate NP contributions to these functions in the VLQ models, using the results obtained in the previous section. To this end it will be useful to adopt the notations of $[35,36]$.

We define the relevant CKM factors by ${ }^{9}$

$$
\lambda_{i j}^{(U)}=V_{U i}^{*} V_{U j} \quad \text { with } \quad U \in\{u, c, t\} \quad \text { and } \quad i, j \in\{d, s, b\} .
$$

We introduce further

$$
g_{\mathrm{SM}}^{2}=4 \frac{G_{F}^{2} M_{W}^{2}}{2 \pi^{2}}=1.78137 \times 10^{-7} \mathrm{GeV}^{-2}
$$

The relevant master functions in the SM are

$$
S_{0}\left(x_{t}\right), \quad X_{0}\left(x_{t}\right), \quad Y_{0}\left(x_{t}\right), \quad Z_{0}\left(x_{t}\right) .
$$

They are flavour universal and real valued. For completeness their explicit expressions can be found in the appendices. In the considered VLQ models new contributions not only break flavour universality, but also bring in new CP-violating phases, so that minimal flavour violation (MFV) is violated.

\section{$4.1|\Delta F|=2$}

The Wilson coefficients ${ }^{10}$ of $|\Delta F|=2$ operators governing neutral kaon and $B_{q}$-meson mixing $(q=d, s)$, defined in appendix C.1, can receive at the scale $\mu_{\mathrm{EW}}$ several contributions depicted in figure 3, depending on the model. Firstly, there are the local contributions, figure 3a, from the one-loop decoupling presented in section 3.2, which are formally of order $v^{2} / M^{2}$, but one-loop suppressed. Secondly, there are also local 1stLLA contributions in $\mathrm{G}_{\mathrm{SM}}$ models due to top-Yukawa RG effects from $\psi^{2} H^{2} D$ operators presented in section 3.3, which are formally of order $v^{2} / M^{2} \ln (v / M)$ and also one-loop suppressed. Thirdly, there are double-insertions of flavour-changing $Z^{(\prime)}$ couplings, figure $3 \mathrm{~b}$, that count due to the double insertion formally as $v^{4} / M^{4}$, but are generated already at tree-level. Fourthly, when considering several VLQ representations also double-insertions of $\psi^{2} \varphi^{3}$-type operators [3], generating flavour-changing neutral Higgs exchange, can contribute in analogy to figure $3 \mathrm{~b}$ when replacing the $Z^{(\prime)}$ by $h^{0}$. As a consequence in this case also non-vanishing contributions can arise to the operators $O_{\mathrm{S} \chi \chi, 1}$ with $\chi=L, R$ and $O_{\mathrm{LR}, 2}$ [32].

\footnotetext{
${ }^{9}$ This notation differs sufficiently from the one for Yukawa couplings $\lambda_{i}$ so that there should not be any problem in distinguishing them.

${ }^{10}$ See footnote 2 .
} 


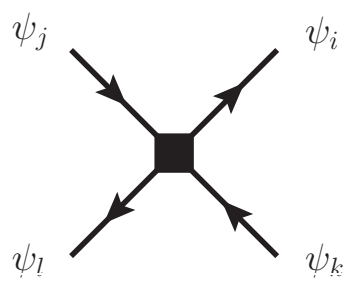

(a)

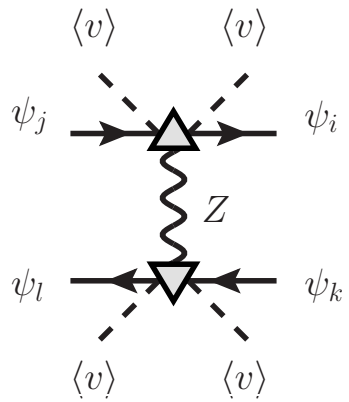

(b)

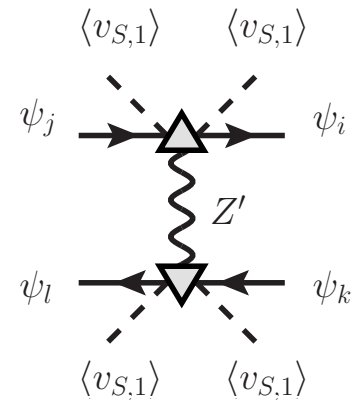

Figure 3. Figure 3a shows flavour-changing four-quark transitions in the $\mathrm{G}_{\mathrm{SM}}^{(\prime)}$-EFT that are mediated by local $\psi^{4}$-operators, generated at the scale $\mu_{M}$ at one-loop level (indicated by the filled square). Figure $3 \mathrm{~b}$ shows contributions from double insertions of $\psi^{2} \varphi^{2} D$-operators via intermediate $Z$ or $Z^{\prime}$ exchange, which are formally of higher power, but are generated by tree-level VLQ exchange (indicated by the triangles).

Unless we consider several VLQ representations simultaneously, new physics contributions from box diagrams, the top-Yukawa generated 1stLLA contributions in LH $\mathrm{G}_{\mathrm{SM}}$ models and the double-insertions of flavour-changing $Z^{(\prime)}$-couplings involve only the operators $O_{\mathrm{VLL}}^{i j}$ and $O_{\mathrm{VRR}}^{i j}$. Below $\mu_{\mathrm{EW}}$, they obey the same RG evolution $(3.36)$ - with appropriate change of number of active quark flavours $N_{f}=6 \rightarrow 5-$ and enter the $M_{12}$ element of the mass-mixing matrix as the linear combination

$$
\left[C_{\mathrm{VLL}}^{i j}+C_{\mathrm{VRR}}^{i j}\right]\left(\mu_{\mathrm{EW}}\right) \equiv S_{i j}=S_{0}\left(x_{t}\right)+\Delta S_{i j}
$$

with $\Delta S_{i j}$ denoting VLQ contributions. The SM contribution is given at LO by $S_{0}\left(x_{t}\right)$, see (C.4). We have

$$
\Delta S_{i j}=\left[\Delta S_{i j}\right]_{\mathrm{VLL}}+\left[\Delta S_{i j}\right]_{\mathrm{VRR}}
$$

although in a given model only one of these contributions is present. If two different models containing LH and RH couplings are combined, the most important transitions in $|\Delta F|=2$ are not these two operators, but $O_{\mathrm{LR}, 1}^{i j}$ and $O_{\mathrm{LR}, 2}^{i j}$.

The $\left[\Delta S_{i j}\right]_{\mathrm{V} \chi \chi}$ with $\chi=L, R$ include quite generally box diagrams with VLQs and scalar exchanges, the top-Yukawa generated 1stLLA contributions in LH $\mathrm{G}_{\mathrm{SM}}$ models as well as tree-level $Z$ and $Z^{\prime}$ contributions. We can therefore write

$$
\left[\Delta S_{i j}\right]_{\mathrm{V} \chi \chi}=\mathcal{C}_{\mathrm{V} \chi \chi}^{i j}\left(\mu_{\mathrm{EW}}\right)+\frac{4 r_{Z}}{g_{\mathrm{SM}}^{2} M_{Z}^{2}}\left[\frac{\Delta_{\chi}^{i j}(Z)}{\lambda_{i j}^{(t)}}\right]^{2}+\frac{4 r_{Z^{\prime}}}{g_{\mathrm{SM}}^{2} M_{Z^{\prime}}^{2}}\left[\frac{\Delta_{\chi}^{i j}\left(Z^{\prime}\right)}{\lambda_{i j}^{(t)}}\right]^{2},
$$

where $\mathcal{C}_{\mathrm{V} \chi \chi}^{i j}\left(\mu_{\mathrm{EW}}\right)$ are given by (3.36) for $\chi=R$ or the sum of (3.36) and (3.30) for $\chi=L$. The $r_{V}$ for $V=Z, Z^{\prime}$ are NLO QCD corrections ${ }^{11}$ to figure $3 \mathrm{~b}$ from decoupling of the $V$ boson at the scale $\mu=\mu_{\mathrm{EW}}$ [38], Note the model-dependence of the factors $\Delta_{\chi}^{i j}(Z)$ and

\footnotetext{
${ }^{11}$ Since we decouple $Z$ and $Z^{\prime}$ simultaneously at $\mu_{\mathrm{EW}} \sim M_{Z}$, we do not resum logarithms between scales $M_{Z^{\prime}}$ and $\mu_{\mathrm{EW}}$ as for example in ref. [37].
} 


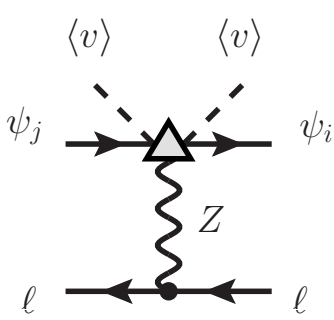

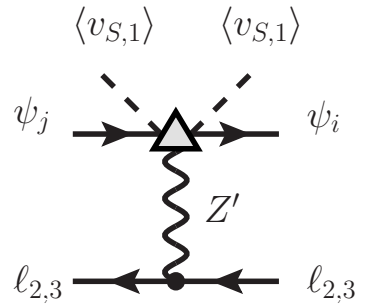

(a)

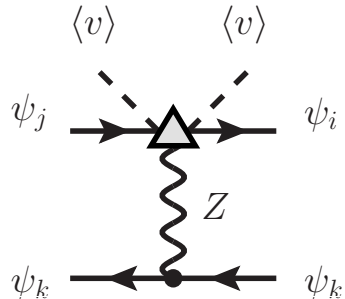

(b)

Figure 4. Flavour-changing $\Delta F=1$ processes that are mediated in the $G_{\mathrm{SM}^{\prime}}^{(\prime}$-EFTs by dimension six $\psi^{2} \varphi^{2} D$-operators (indicated by the triangle), which are in turn generated at the scale $\mu_{M}$ at tree level. Semileptonic transitions $\psi_{j} \rightarrow \psi_{i} \ell \bar{\ell}$ in figure 4 a can be mediated by both $Z$ and $Z^{\prime}$ exchange, depending on the model. Not shown are analogous transitions $\psi_{j} \rightarrow \psi_{i} \nu \bar{\nu}$. Note that the $Z^{\prime}$ couples only to the second and third generations of leptons and neutrinos. Hadronic transitions $\psi_{j} \rightarrow \psi_{i} \psi_{k} \bar{\psi}_{k}$ in figure $4 \mathrm{~b}$ are mediated only by $Z$ exchange, with $\psi=\left(q_{L}, u_{R}, d_{R}\right)$, depending on the operator.

$\Delta_{\chi}^{i j}\left(Z^{\prime}\right)$, given in table 3 and table 4 , and the different dependence on the VLQ mass of these factors and $\mathcal{C}_{\mathrm{V} \chi \chi}^{i j}\left(\mu_{\mathrm{EW}}\right)$.

The top-Yukawa operator mixing generates in RH $\mathrm{G}_{\mathrm{SM}}$ models also LR operators for a single VLQ representation. When two or more representations are considered, also LR and SLL (SRR) operators contribute in principle. The Wilson coefficients of LR operators can receive contributions from box diagrams, top-Yukawa generated RG effects and tree-level $Z^{(\prime)}$ exchanges, whereas SLL (SRR) and LR,2 from tree-level $h^{0}$ exchange. The results for all box contributions $\mathcal{C}_{\mathrm{LR}, 1}^{i j}$ are given in formulae (3.18) and (3.21) and the RG evolution in (3.36), to which the top-Yukawa generated 1stLLA contributions (3.30) have to be added in $\mathrm{RH} \mathrm{G}_{\mathrm{SM}}$ models. Adding the $Z$ - and $Z^{\prime}$-contributions, one arrives at

$$
\begin{aligned}
& C_{\mathrm{LR}, 1}^{i j}\left(\mu_{\mathrm{EW}}\right)=\mathcal{C}_{\mathrm{LR}, 1}^{i j}\left(\mu_{\mathrm{EW}}\right)+\frac{1}{\mathcal{N}_{i j}}\left[\frac{\Delta_{L}^{i j}(Z) \Delta_{R}^{i j}(Z)}{M_{Z}^{2}}+\frac{\Delta_{L}^{i j}\left(Z^{\prime}\right) \Delta_{R}^{i j}\left(Z^{\prime}\right)}{M_{Z^{\prime}}^{2}}\right], \\
& C_{\mathrm{LR}, 2}^{i j}\left(\mu_{\mathrm{EW}}\right)=\mathcal{C}_{\mathrm{LR}, 2}^{i j}\left(\mu_{\mathrm{EW}}\right)
\end{aligned}
$$

with the couplings $\Delta_{\chi}^{i j}\left(Z^{(\prime)}\right)(\chi=L, R)$ collected in table 3 and table $4 . \mathcal{N}_{i j}$ is defined in (C.2).

The RG evolution from $\mu_{\mathrm{EW}}$ to $m_{b}$ is done at NLLA accuracy for the SM contribution and LLA accuracy for the VLQ contribution.

\section{$4.2|\Delta F|=1:$ semi-leptonic $d_{j} \rightarrow d_{i}+(\ell \bar{\ell}, \nu \bar{\nu})$}

Semileptonic decays in the down-quark sector receive in VLQ models contributions via the $Z$ and $Z^{\prime}$ tree-level exchanges depicted in figure $4 \mathrm{a}$. They lead to modifications of the Wilson coefficients of the corresponding phenomenological EFTs of $d_{j} \rightarrow d_{i} \nu \bar{\nu}$ and $d_{j} \rightarrow$ $d_{i} \ell \bar{\ell}$ decays given in appendix C.2 and appendix C.3, respectively. All Wilson coefficients 
in this section are formally at $\mu_{\mathrm{EW}}$, but since the corresponding operators are conserved currents under QCD, the RG evolution to the scale $\mu_{b}$ is trivial in all cases. ${ }^{12}$

The $V=Z, Z^{\prime}$ contributions modify the Wilson coefficients and one-loop functions

$$
C_{L(R)}^{i j, \nu}=-\sum_{V} \frac{X_{L(R)}^{i j, \nu}(V)}{s_{W}^{2}}, \quad \quad X_{L(R)}^{i j, \nu}(V)=\frac{\Delta_{L}^{\nu \bar{\nu}}(V)}{g_{\mathrm{SM}}^{2} M_{V}^{2}} \frac{\Delta_{L(R)}^{i j}(V)}{\lambda_{i j}^{(t)}},
$$

which enter the expressions for $d_{j} \rightarrow d_{i} \bar{\nu} \nu$ decays like $K^{+} \rightarrow \pi^{+} \nu \bar{\nu}, K_{L} \rightarrow \pi^{0} \nu \bar{\nu}$ and also $B \rightarrow K^{(*)} \nu \bar{\nu}$ with more details in appendix C.2.

The Wilson coefficients of the operators entering the $d_{j} \rightarrow d_{i} \ell \bar{\ell}$ transitions receive the following contributions

$$
\begin{aligned}
C_{9\left(9^{\prime}\right)}^{i j, \ell} & =-\sum_{V} \frac{\left[\Delta_{R}^{\ell \bar{\ell}}(V)+\Delta_{L}^{\ell \bar{\ell}}(V)\right]}{s_{W}^{2} g_{\mathrm{SM}}^{2} M_{V}^{2}} \frac{\Delta_{L(R)}^{i j}(V)}{\lambda_{i j}^{(t)}}, \\
C_{10\left(10^{\prime}\right)}^{i j, \ell} & =-\sum_{V} \frac{\left[\Delta_{R}^{\ell \bar{\ell}}(V)-\Delta_{L}^{\ell \bar{\ell}}(V)\right]}{s_{W}^{2} g_{\mathrm{SM}}^{2} M_{V}^{2}} \frac{\Delta_{L(R)}^{i j}(V)}{\lambda_{i j}^{(t)}},
\end{aligned}
$$

where the leptonic $Z$ couplings are taken to be the ones of the SM except for $\mathrm{G}_{\mathrm{SM}}^{\prime}(\Phi)$ models, where $Z-Z^{\prime}$ mixing is included following (3.11). There are no $Z^{\prime}$ contributions to $C_{10\left(10^{\prime}\right)}$, as the lepton couplings are vectorial, see (3.10).

The purely leptonic decay $K_{L} \rightarrow \mu \bar{\mu}$ is described by $(\bar{s} \rightarrow \bar{d})$

$$
Y_{\mathrm{A}}(K)=Y_{L}^{\mathrm{SM}}+\frac{\left[\Delta_{R}^{\mu \bar{\mu}}(Z)-\Delta_{L}^{\mu \bar{\mu}}(Z)\right]}{g_{\mathrm{SM}}^{2} M_{Z}^{2}}\left[\frac{\Delta_{L}^{s d}(Z)-\Delta_{R}^{s d}(Z)}{\lambda_{s d}^{(t)}}\right],
$$

with $Y_{L}^{\mathrm{SM}}=0.942[39]$.

\section{3 $|\Delta F|=1$ : hadronic $d_{j} \rightarrow d_{i} q \bar{q}$ and $\varepsilon^{\prime} / \varepsilon$}

Purely hadronic flavour-changing decays $d_{j} \rightarrow d_{i} q \bar{q}$ receive in the considered VLQ models predominantly contributions from $Z$ exchange depicted in figure $4 \mathrm{~b}$. Other contributions from scalar boxes, figure $3 \mathrm{a}$, or double-insertions of $Z$ or $Z^{\prime}$ exchange in figure $3 \mathrm{~b}$ are either loop- or power-suppressed. The phenomenological EFT of these transitions is given in appendix C.4. Since the flavour-diagonal $Z$ couplings are given by the SM ones to the order we are working in, no dependence on $q$ arises. The non-vanishing contributions to the $|\Delta F|=1$ Wilson coefficients are conveniently rewritten as NP contributions to the Inami-Lim $Z$-penguin function $C$ (see appendices C.2 and C.3) ${ }^{13}$

$$
C_{L(R)}^{i j}=-\frac{g_{Z}}{2 g_{\mathrm{SM}}^{2} M_{Z}^{2}} \frac{\Delta_{L(R)}^{i j}(Z)}{\lambda_{i j}^{(u)}} .
$$

\footnotetext{
${ }^{12}$ The usual mixing of $Q_{9}$ operators with current-current operators $Q_{1,2}$ present in the SM and affecting $C_{9}$ coefficient is fully negligible here because NP contributions to $C_{1,2}$ are tiny in all models.

${ }^{13}$ Note that whereas the SM contribution to the function $C$ is gauge dependent this shift is gauge independent.
} 
It contributes at the scale $\mu_{\mathrm{EW}}$ to the Wilson coefficients of the QCD- and EW-penguin operators [40],

$$
C_{3\left(5^{\prime}\right)}^{i j}=\frac{\alpha}{6 \pi} \frac{C_{L(R)}^{i j}}{s_{W}^{2}}, \quad C_{7\left(9^{\prime}\right)}^{i j}=\frac{\alpha}{6 \pi} 4 C_{L(R)}^{i j}, \quad C_{9\left(7^{\prime}\right)}^{i j}=-\frac{\alpha}{6 \pi} \frac{c_{W}^{2}}{s_{W}^{2}} 4 C_{L(R)}^{i j} .
$$

The RG evolution induces also non-vanishing contributions for the remaining QCD- and EW-penguin operators at lower scales relevant for Kaon and $B$-meson decays. Here we are mainly interested in $\mathrm{CP}$ violation in the Kaon sector, especially $\varepsilon^{\prime} / \varepsilon$.

It is known from various analyses of $\varepsilon^{\prime} / \varepsilon$, see [40] and references therein, that NP has to generate contributions to the Wilson coefficients of $O_{8} \propto(V-A) \otimes(V+A)$ or $O_{8}^{\prime} \propto$ $(V+A) \otimes(V-A)$ operators at the low energy scale in order to be able to modify significantly the SM predictions. This requires the presence of both LH flavour-violating couplings and $\mathrm{RH}$ flavour-diagonal couplings of $Z$ or $Z^{\prime}$ in the case of $O_{8}$, or RH flavour-violating couplings and LH flavour-diagonal couplings in the case of $O_{8^{\prime}}$. But in the models considered quark couplings of the $Z^{\prime}$ are either $\mathrm{LH}$ or $\mathrm{RH}$, hence such contributions can only be generated as a higher-order effect. Given that $(V-A)$ and $(V+A)$ flavour-diagonal $Z$ couplings to SM quarks are always present, tree-level $Z$ exchanges fully dominate. NP contributions to $O_{9,10} \propto(V-A) \otimes(V-A)$ or $O_{9,10}^{\prime} \propto(V+A) \otimes(V+A)$ operators are negligible due to their suppressed hadronic matrix elements relative to the ones of $O_{8}$ and $O_{8}^{\prime}$. This can be clearly seen in the semi-numeric expression (C.29) for $\varepsilon^{\prime} / \varepsilon$, where the coefficients of $C_{7}^{(\prime)}$, which mixes into $C_{8}^{(\prime)}$, is largely enhanced w.r.t. all others. Whether $O_{8}$ or $O_{8}^{\prime}$ is generated depends on whether a given model has $(V-A)$ or $(V+A)$ flavour-violating couplings:

- Within the $\mathrm{G}_{\mathrm{SM}^{-}}$and $\mathrm{G}_{\mathrm{SM}}^{\prime}(\Phi)$-models, the pattern of NP contributions to $\varepsilon^{\prime} / \varepsilon$ is as follows

$$
\begin{aligned}
& \text { singlets : } D \rightarrow\left(O_{8}\right) \text {, } \\
& \text { doublets: } Q_{V}, Q_{d} \rightarrow\left(O_{8}^{\prime}\right) \text {, } \\
& \text { triplets: } \quad T_{d}, \quad T_{u} \rightarrow\left(O_{8}\right) \text {. }
\end{aligned}
$$

- In $\mathrm{G}_{\mathrm{SM}}^{\prime}(S)$-models $\varepsilon^{\prime} / \varepsilon$ remains SM-like, which could become problematic as we discuss briefly below.

Tree-level $Z$ contributions to $\varepsilon^{\prime} / \varepsilon$ have been recently considered in detail in ref. [40], where explicit expressions for the relevant hadronic matrix elements $\left\langle Q_{8}\left(m_{c}\right)\right\rangle_{2}$ and $\left\langle Q_{8}^{\prime}\left(m_{c}\right)\right\rangle_{2}$ can be found. Whereas these matrix elements differ only by sign from each other, their Wilson coefficients differ also in magnitude, the one of $Q_{8}^{\prime}$ being larger by a factor of $c_{W}^{2} / s_{W}^{2}=3.33$. This can also be seen in eq. (4.13), remembering that the Wilson coefficients of $Q_{8}$ and $Q_{8}^{\prime}$ at $\mu=m_{c}$ are directly related to the Wilson coefficients of $Q_{7}$ and $Q_{7}^{\prime}$ at $\mu_{\mathrm{EW}}$, respectively.

Finally let us mention that the top-Yukawa generated 1stLLA contributions to $|\Delta F|=$ 1 operators in $\mathrm{G}_{\mathrm{SM}}$ models discussed in section 3.3 induce operators with the same chiral structure as already present from the $Z$-exchange due to $\psi^{2} H^{2} D$ operators. In particular the $\psi^{2} H^{2} D$ Wilson coefficients generate $\psi^{4}$ Wilson coefficients via the mixing given 
in (B.23)-(B.28)

$$
\begin{aligned}
\mathcal{C}_{H q}^{(1,3)} & \rightarrow \mathcal{C}_{q q}^{(1,3)}, \mathcal{C}_{q u}^{(1)} \\
\mathcal{C}_{H d} & \rightarrow \mathcal{C}_{q d}^{(1)}, \mathcal{C}_{u d}^{(1)}
\end{aligned}
$$

where $\mathcal{O}_{q q}^{(1,3)} \sim(V-A) \otimes(V-A), \mathcal{O}_{q u, q d}^{(1)} \sim(V-A) \otimes(V+A)$ and $\mathcal{O}_{u d}^{(1)} \sim(V+A) \otimes(V+A)$. Given their additional suppression w.r.t. existing contributions we do not consider these contributions further.

The status of $\varepsilon^{\prime} / \varepsilon$ in the SM can be summarized as follows. The RBC-UKQCD lattice collaboration calculating hadronic matrix elements of all operators, but not including isospin-breaking effects, finds [19, 41]

$$
\left(\varepsilon^{\prime} / \varepsilon\right)_{\mathrm{SM}}=(1.38 \pm 6.90) \times 10^{-4} \quad(\mathrm{RBC}-\mathrm{UKQCD}) .
$$

Using the hadronic matrix elements of QCD- and EW-penguin $(V-A) \otimes(V+A)$ operators from RBC-UKQCD lattice collaboration $[19,41]$ but extracting the matrix elements of $(V-A) \otimes(V-A)$-penguin operators from the CP-conserving $K \rightarrow \pi \pi$ amplitudes and including isospin breaking effects, one finds [20]

$$
\left(\varepsilon^{\prime} / \varepsilon\right)_{\mathrm{SM}}=(1.9 \pm 4.5) \times 10^{-4} \quad(\mathrm{BGJJ}) .
$$

This result differs by $2.9 \sigma$ from the experimental world average from the NA48 [42] and $\mathrm{KTeV}[43,44]$ collaborations,

$$
\left(\varepsilon^{\prime} / \varepsilon\right)_{\exp }=(16.6 \pm 2.3) \times 10^{-4},
$$

suggesting that models providing enhancement of $\varepsilon^{\prime} / \varepsilon$ are favoured. A new analysis in ref. [22] confirms these findings

$$
\left(\varepsilon^{\prime} / \varepsilon\right)_{\mathrm{SM}}=(1.1 \pm 5.1) \times 10^{-4} \quad(\mathrm{KNT}) .
$$

These results are supported by upper bounds on the matrix elements of the dominant penguin operators from the large- $N_{c}$ dual-QCD approach [21, 45], which allows to derive an upper bound on $\varepsilon^{\prime} / \varepsilon[20]$,

$$
\left(\varepsilon^{\prime} / \varepsilon\right)_{\mathrm{SM}} \leq(8.6 \pm 3.2) \times 10^{-4},
$$

still $2 \sigma$ below the experimental data. In particular it has been demonstrated in ref. [45] that final state interactions are much less relevant for $\varepsilon^{\prime} / \varepsilon$ than previously claimed in refs. [4653]. These findings diminish significantly hopes that improved lattice QCD calculations will be able to bring the SM prediction for $\varepsilon^{\prime} / \varepsilon$ to agree with the experimental data in (4.19), motivating additionally to search for NP models capable of alleviating this tension.

In fact it has been demonstrated that in general models with flavour-changing $Z$ and $Z^{\prime}$ exchanges [40, 54], in the Littlest Higgs model with $T$-parity [55], 331 models [56, 57] and supersymmetric models [58-60] agreement with the data for $\varepsilon^{\prime} / \varepsilon$ can be obtained, with interesting implications for other flavour observables.

We will see in section 6 that also in VLQ models large NP contributions to $\varepsilon^{\prime} / \varepsilon$ are possible, such that agreement with the data in (4.19) can be obtained with a significant impact not only on rare $K$ decays but also $B$ decays. 


\section{$5 \quad$ Patterns of flavour violation}

Our analysis involves three model variants $\mathrm{G}_{\mathrm{SM}}, \mathrm{G}_{\mathrm{SM}}^{\prime}(S)$ and $\mathrm{G}_{\mathrm{SM}}^{\prime}(\Phi)$, with up to five VLQ representations. In this section we describe the patterns of flavour violation in $|\Delta F|=1,2$ FCNC processes in the Kaon and $B_{d, s}$-meson sectors that can be expected in these models, based on our results in sections 3 and 4 . The quantitative phenomenology depends in addition to the NP parameters on the CKM and hadronic ones and will be discussed in the next section. However, on the basis of the information collected so far, some general patterns of flavour violation emerge and it is possible to state whether in a given model relevant NP contributions to a given observable can be expected. We hope that the collection of observations below will be useful in monitoring the numerical analysis of the next section.

\section{$5.1|\Delta F|=2$}

In all models local VLQ contributions to $|\Delta F|=2$ operators are generated at the VLQscale $\mu_{M}$ via one-loop box diagrams. The contributions from tree-level exchanges of $Z$ and $Z^{\prime}$ at the scale $\mu_{\mathrm{EW}}$ are power-suppressed due to the hierarchy (3.1) and should be therefore numerically subleading, at least for large VLQ masses. This property decouples $|\Delta F|=1$ and $|\Delta F|=2$ contributions to some extent, rendering it easier to accommodate potential tensions $[61,62]$ in $\Delta F=2$ processes.

In $\mathrm{G}_{\mathrm{SM}}$ models additional contributions from four-fermion operators are generated through Yukawa RG evolution from $\mu_{M}$ to $\mu_{\mathrm{EW}}$. In the case of models $Q_{V}$ and $Q_{d}$ these contributions turn out to be dominant for $\mu_{M} \geq 1 \mathrm{TeV}$ in the $K$ meson system and very important in the $B_{d, s}$ meson systems. In the following we compare the various contributions one by one.

The $|\Delta F|=2$ box contributions given in eq. (3.18) and (3.21) depend only on the VLQ mass(es) $M$ and their Yukawa couplings $\lambda_{i}^{\mathrm{VLQ}}$, but neither on the gauge couplings nor on the scalar sector. Moreover for a given VLQ-representation, they are equal in $\mathrm{G}_{\mathrm{SM}}$ and $\mathrm{G}_{\mathrm{SM}}^{\prime}(\Phi)$ models owing to the equality of (2.5) and (2.7) upon $H \leftrightarrow \Phi$. Hence the measurements of $|\Delta F|=2$ observables will result for a given $M$ in the very same constraints on $\lambda_{i}^{\mathrm{VLQ}}$ in both $\mathrm{G}_{\mathrm{SM}}$ and $\mathrm{G}_{\mathrm{SM}}^{\prime}(\Phi)$ models.

Using (4.6), the relative size of box-to- $Z$ exchange in $\mathrm{G}_{\mathrm{SM}}$ and $\mathrm{G}_{\mathrm{SM}}^{\prime}(\Phi)$ models is

$$
\frac{(\Delta S)_{\mathrm{Box}}}{(\Delta S)_{Z}}=a \eta_{L L} \frac{g_{Z}^{2}}{8 \pi^{2}}\left[\frac{\eta_{6}^{2 / 7}}{r_{Z}}\right] \frac{M^{2}}{M_{Z}^{2}} \times\left\{\begin{array}{ll}
1 & \mathrm{G}_{\mathrm{SM}} \\
c_{\beta}^{-4} & \mathrm{G}_{\mathrm{SM}}^{\prime}(\Phi)
\end{array},\right.
$$

with $\eta_{L L}$ collected in table $5, r_{Z} \approx 1$, and $a=4$ for $T_{d}$ and unity otherwise. While the $Z$ contribution is comparable to the box contribution for $M \approx 1-2 \mathrm{TeV}$, it amounts only to a few percent for $M=10 \mathrm{TeV}$ in $\mathrm{G}_{\mathrm{SM}}$ models, whereas in $\mathrm{G}_{\mathrm{SM}}^{\prime}(\Phi)$ models the $Z$-contributions are suppressed by $c_{\beta}^{4}$. In $\mathrm{G}_{\mathrm{SM}}^{\prime}(\Phi)$ models we have furthermore

$$
\frac{(\Delta S)_{Z^{\prime}}}{(\Delta S)_{Z}}=\left(r^{\prime}\right)^{2}\left[\frac{r_{Z^{\prime}}}{r_{Z}}\right] \frac{M_{Z}^{2}}{M_{Z^{\prime}}^{2}}, \quad \mathrm{G}_{\mathrm{SM}}^{\prime}(\Phi)
$$


with $r_{Z^{\prime}} \approx r_{Z} \approx 1$. Therefore $Z$ exchange might be more important w.r.t. the $Z^{\prime}$ contribution for $M_{Z}<M_{Z^{\prime}}$, depending on $r^{\prime}$, see (3.13) but both are suppressed w.r.t. the box contribution.

In the $\mathrm{G}_{\mathrm{SM}}^{\prime}(S)$ models the same picture holds qualitatively, however a $Z$-exchange is absent and the relative size of box-to- $Z^{\prime}$ exchange is different,

$$
\frac{(\Delta S)_{\mathrm{Box}}}{(\Delta S)_{Z^{\prime}}}=\frac{\left(X g^{\prime}\right)^{2}}{(4 \pi)^{2}}\left[\frac{\eta_{6}^{2 / 7}}{r_{Z^{\prime}}}\right] \frac{M^{2}}{M_{Z^{\prime}}^{2}}, \quad \mathrm{G}_{\mathrm{SM}}^{\prime}(S)
$$

which for $X=1$ reduces to the result in ref. [9]. In contrast to $\mathrm{G}_{\mathrm{SM}}$ and $\mathrm{G}_{\mathrm{SM}}^{\prime}(\Phi)$ models, we note the particular structure of $Z^{\prime}$ couplings, not being suppressed by $M_{Z}^{2} / M_{Z^{\prime}}^{2}$. A lower bound on $|X| v_{S}=M_{Z^{\prime}} / g^{\prime} \gtrsim 750 \mathrm{GeV}$ exists in $\mathrm{G}_{\mathrm{SM}}^{\prime}(S)$ models, mainly from a combination of $Z \rightarrow 4 \mu$ and the neutrino trident production [9]. This implies that only for $M \gtrsim 9 \mathrm{TeV}$ the ratio $(\Delta S)_{\mathrm{Box}} /(\Delta S)_{Z^{\prime}} \gtrsim 1$ and shows the numerical importance of the $Z^{\prime}$ contributions, unless one considers much larger VLQ masses.

With only these contributions taken into account the $|\Delta F|=2$ observables are not sensitive to the chirality of the VLQ interactions as long as only one VLQ representation is present, because the contributions are additive as can be seen in (4.5). However, the inclusion of RG Yukawa effects and NLO contributions discussed in [18] changes this picture drastically in the case of $\mathrm{G}_{\mathrm{SM}}$ models with flavour changing $\mathrm{RH}$ currents $\left(Q_{d}, Q_{V}\right)$ and has also significant impact in the remaining three models with LH currents.

In the case of $D, T_{d}$ and $T_{u}$ models we find

$$
\left[\frac{(\Delta S)_{\mathrm{RG}}}{(\Delta S)_{\mathrm{Box}}}\right]^{i j}=\frac{\kappa_{m}}{\eta_{m m}} \frac{\lambda_{i j}^{t}}{\Lambda_{i j}^{m}} \frac{2 m_{t}^{2}}{v^{2} \eta_{6}^{2 / 7}}\left[\ln \frac{\mu_{M}}{\mu_{\mathrm{EW}}}+\frac{F_{\mathrm{NLO}}\left(x_{t}, \mu_{\mathrm{EW}}\right)}{\kappa_{m} \Lambda_{i j}^{m}}\right]
$$

with $\kappa_{m}$ given in (3.32) and $\eta_{m m}$ in table 5. The NLO correction

$$
\begin{aligned}
F_{\mathrm{NLO}}\left(x_{t}, \mu_{\mathrm{EW}}\right)= & {\left[\mathcal{C}_{H q}^{(1)}\right]_{i j} H_{1}\left(x_{t}, \mu_{\mathrm{EW}}\right)-\left[\mathcal{C}_{H q}^{(3)}\right]_{i j} H_{2}\left(x_{t}, \mu_{\mathrm{EW}}\right) } \\
& +\frac{2 S_{0}\left(x_{t}\right)}{x_{t}} \sum_{m}\left(\lambda_{t}^{i m}\left[\mathcal{C}_{H q}^{(3)}\right]_{m j}+\left[\mathcal{C}_{H q}^{(3)}\right]_{i m} \lambda_{t}^{m j}\right)
\end{aligned}
$$

has been calculated in [18], where also the $x_{t}$-dependent functions $H_{1,2}$ can be found. The result for $H_{1}\left(x_{t}, \mu_{\mathrm{EW}}\right)$ in [18] has been confirmed in [63] where NLO corrections in the context of a general analysis of $Z$-mediated NP have been calculated, however in contrast to [18] leaving out $\mathrm{RG}$ effects above the electroweak scale represented by $\ln \mu_{M} / \mu_{\mathrm{EW}}$ in (5.4) and (5.6).

In the case of $Q_{d}$ and $Q_{V}$ models the box and RG contributions yield coefficients to different operators, hence a meaningful comparison of their impact on observables has to include their QCD running between $\mu_{\mathrm{EW}}$ and the light flavour scales (we choose $3 \mathrm{GeV}$ for Kaons and $M_{B}$ for $\left.B_{d, s}\right)$ as well as the corresponding matrix elements. We find

$$
\left[\frac{\left(M_{12}^{*}\right)_{\mathrm{RG}}}{\left(M_{12}^{*}\right)_{\mathrm{Box}}}\right]^{i j}=\left[\frac{\left(M_{12}^{*}\right)_{\mathrm{LR}}}{\left(M_{12}^{*}\right)_{\mathrm{VRR}}^{\mathrm{box}}}\right]^{i j}=\frac{\kappa_{m}}{\eta_{m m}} \frac{\lambda_{i j}^{t}}{\Lambda_{i j}^{m}} \frac{2 m_{t}^{2}}{v^{2} \eta_{6}^{2 / 7}}\left[\ln \frac{\mu_{M}}{\mu_{\mathrm{EW}}}+H_{1}\left(x_{t}, \mu_{\mathrm{EW}}\right)\right] R^{i j},
$$


with $R^{i j}$ including $\mathrm{RG}$ factors and the ratio of the hadronic matrix elements. From eqs. (60) and (61) in [18] we obtain

$$
R^{s d} \approx-80 \quad \text { and } \quad R^{b(d, s)} \approx-3 .
$$

This large chiral enhancement in the Kaon system renders the RG contribution dominant, while in the $B_{d, s}$ systems the contribution remains comparable with the box contribution.

\section{$5.2|\Delta F|=1$}

In semi-leptonic $|\Delta F|=1$ processes governed by $d_{j} \rightarrow d_{i}+(\ell \bar{\ell}, \nu \bar{\nu})$, the VLQ contributions arise from tree-level $Z$ exchange in $\mathrm{G}_{\mathrm{SM}}$ models, $Z^{\prime}$ exchange in $\mathrm{G}_{\mathrm{SM}}^{\prime}(S)$ models and both in $\mathrm{G}_{\mathrm{SM}}^{\prime}(\Phi)$ models.

It is instructive to begin the discussion with $\mathrm{G}_{\mathrm{SM}}^{\prime}(S)$ models considered already in ref. [9], as they involve only $Z^{\prime}$ contributions to $\Delta F=1$ processes and the leptonic $Z^{\prime}$ couplings have a special structure as given in eq. (3.10). Moreover, as pointed out in that paper, the $|\Delta F|=1$ contributions of VLQs in these models are independent of the scalarand gauge-sector parameters, in contrast to $|\Delta F|=2$ contributions that depend on $v_{S}$. We find the following pattern in NP contributions:

- Due to the equality of the LH and RH $Z^{\prime}$ couplings to leptons in (3.10), $Z^{\prime}$ exchange does neither contribute to $B_{s, d} \rightarrow \mu \bar{\mu}$ nor to $K_{L} \rightarrow \mu \bar{\mu}$. If future improved data will show the need for NP contributions to $B_{s, d} \rightarrow \mu \bar{\mu}$, this will be a problem for this scenario.

- The crucial virtue of $\mathrm{G}_{\mathrm{SM}}^{\prime}(S)$ models, pointed out in [9], is the possibility of solving the LHCb anomalies; in particular, they can accommodate violation of lepton-flavour universality (LFU).

- In $B \rightarrow K\left(K^{*}\right) \nu \bar{\nu}$ only small contributions are possible due to cancellations among muon and tau contributions when averaging over neutrino flavours as a consequence of the $\mathrm{U}(1)_{\mathrm{L}_{\mu}-\mathrm{L}_{\tau}}$ symmetry.

- These cancellations are less efficient in $K^{+} \rightarrow \pi^{+} \nu \bar{\nu}$ due to interference with the charm component, see appendix C.2.

Considering next $\mathrm{G}_{\mathrm{SM}}$ and $\mathrm{G}_{\mathrm{SM}}^{\prime}(\Phi)$ models in which tree-level $Z$ contributions to $\Delta F=$ 1 processes dominate, the most notable feature comes from the tree-level decoupling of the VLQs depicted in figure 1b, which implies a relationship between the flavour-changing $Z$ and $Z^{\prime}$ couplings in these models, again owing to the equality of (2.5) and (2.7) upon $H \leftrightarrow \Phi$. Below the scale $\mu_{M}$ in both models a $\psi^{2} \varphi^{2} D$ operator is generated, with the same Wilson coefficient, where $\varphi=H, \Phi$ in $\mathrm{G}_{\mathrm{SM}}$ and $\mathrm{G}_{\mathrm{SM}}^{\prime}(\Phi)$ models, respectively. The covariant derivative is the same in both models, up to the additional $\mathrm{U}(1)_{\mathrm{L}_{\mu}-\mathrm{L}_{\tau}}$ part in $\mathrm{G}_{\mathrm{SM}}^{\prime}(\Phi)$ models. Upon spontaneous symmetry breaking at the scale $\mu_{\mathrm{EW}}$, this operator becomes $\propto v^{2}$ in $\mathrm{G}_{\mathrm{SM}}$ models and $\propto v_{1}^{2}=c_{\beta}^{2} v^{2}$ in $\mathrm{G}_{\mathrm{SM}}^{\prime}(\Phi)$ models. Consequently, in $\mathrm{G}_{\mathrm{SM}}^{\prime}(\Phi)$ models all $Z$ and $Z^{\prime}$ couplings $\propto c_{\beta}^{2} \Delta^{i j}$ are suppressed by $c_{\beta}^{2}=\left(1+\tan ^{2} \beta\right)^{-1}$ w.r.t. $Z$ couplings $\propto \Delta^{i j}$ in $\mathrm{G}_{\mathrm{SM}}$ models, see (3.12), (3.9) and table 4. 
Note that the additional modifications from $Z-Z^{\prime}$ mixing in $\mathrm{G}_{\mathrm{SM}}^{\prime}(\Phi)$ models do not affect the dependence on the $\lambda_{i}^{\mathrm{VLQ}}$. The suppression by $c_{\beta}^{2}$ can be only softened by going to very small $\tan \beta$. In order to guarantee perturbativity of the top-quark Yukawa coupling $0.3 \lesssim \tan \beta$ [64]. In appendix A.2 we discuss further constraints on $\tan \beta$ in $\mathrm{G}_{\mathrm{SM}}^{\prime}(\Phi)$ models from the measured $Z$ mass and partial widths to leptons, which for $M_{Z}<M_{Z^{\prime}}$ allow at most $2 \lesssim \tan \beta$, i.e. $c_{\beta}^{2} \lesssim 0.2$. Depending on the choice of $g^{\prime}$ and $v_{S}$, this bound becomes even stronger. Therefore, VLQ effects in $|\Delta F|=1$ FCNC processes are generically suppressed in $\mathrm{G}_{\mathrm{SM}}^{\prime}(\Phi)$ models w.r.t. $\mathrm{G}_{\mathrm{SM}}$ models. As an example one might consider the Wilson coefficient $C_{9}^{i j}$ given in (4.9), governing $d_{j} \rightarrow d_{i} \ell \bar{\ell}$. The suppression factor in $\mathrm{G}_{\mathrm{SM}}^{\prime}(\Phi)$ versus $\mathrm{G}_{\mathrm{SM}}$ models is

$$
\frac{\left(C_{9}^{i j}\right)_{\mathrm{G}_{\mathrm{SM}}^{\prime}(\Phi)}}{\left(C_{9}^{i j}\right)_{\mathrm{G}_{\mathrm{SM}}}}=c_{\beta}^{2}\left[1-r^{\prime} \xi_{Z Z^{\prime}}-\frac{g^{\prime}}{g_{Z}} \frac{4 Q_{\ell}^{\prime}}{\left(1-4 s_{W}^{2}\right)} \xi_{Z Z^{\prime}}-\frac{g^{\prime}}{g_{Z}} \frac{4 Q_{\ell}^{\prime}}{\left(1-4 s_{W}^{2}\right)} \frac{M_{Z}^{2}}{M_{Z^{\prime}}^{2}}\right] .
$$

The mixing angle $\xi_{Z Z^{\prime}} \sim M_{Z}^{2} / M_{Z^{\prime}}^{2}$ is small in most of the parameter space, such that $(1-$ $\left.4 s_{W}^{2}\right)^{-1} \sim 10$ is overcompensated. The comparison of the first three terms with the last one in the brackets also shows the relative size of the $Z^{\prime}$ to $Z$ contribution in $\mathrm{G}_{\mathrm{SM}}^{\prime}(\Phi)$ models, which is also suppressed by $M_{Z}^{2} / M_{Z^{\prime}}^{2}$. Consequently VLQ contributions to semileptonic $|\Delta F|=1$ FCNC decays are in most cases suppressed in $\mathrm{G}_{\mathrm{SM}}^{\prime}(\Phi)$ w.r.t. $\mathrm{G}_{\mathrm{SM}}$ models.

However, there are exceptions related to the fact that with the parametric suppression of the $Z$ and $Z^{\prime}$ couplings, the values of Yukawa couplings are weaker constrained by $\Delta F=1$ transitions than in $\mathrm{G}_{\mathrm{SM}}$ models and the constraints on Yukawas are governed this time by $\Delta F=2$ processes. A detailed numerical analysis in the next section then shows that the allowed NP effects in $\Delta M_{K}$ are in fact significantly larger than in $\mathrm{G}_{\mathrm{SM}}$ models.

For a given flavour-changing transition the correlations between different $|\Delta F|=1$ observables depend on whether $Z^{(\prime)}$ have LH or RH flavour-violating quark couplings and the size of the corresponding leptonic $Z^{(\prime)}$ couplings. A summary is given in table 6, where in addition to $\mathrm{G}_{\mathrm{SM}}$ and $\mathrm{G}_{\mathrm{SM}}^{\prime}(\Phi)$ models we include $\mathrm{G}_{\mathrm{SM}}^{\prime}(S)$ models discussed already above. The generically small NP contributions in $C_{9}^{(\prime) i j, \ell}$ compared to $C_{10}^{(\prime) i j, \ell}$ and $C_{L(R)}^{i j, \nu}$ in $\mathrm{G}_{\mathrm{SM}}$ models are due to the smallness of leptonic vector $Z$ couplings relative to the axial-vector ones. The additional generic suppression of NP effects in $\mathrm{G}_{\mathrm{SM}}^{\prime}(\Phi)$ w.r.t. $\mathrm{G}_{\mathrm{SM}}$ is due to the aforementioned suppression by $c_{\beta}^{2}$.

We observe that in $\mathrm{G}_{\mathrm{SM}}$ models significant NP effects in $K^{+} \rightarrow \pi^{+} \nu \bar{\nu}, K_{L} \rightarrow \pi^{0} \nu \bar{\nu}$, $B_{s, d} \rightarrow \mu \bar{\mu}, B \rightarrow K^{(*)} \mu \bar{\mu}$ and $B \rightarrow K^{(*)} \nu \bar{\nu}$ are possible, but the LHCb anomalies in angular observables in $B \rightarrow K^{*} \mu \bar{\mu}$ cannot be explained in these models because the vector coupling of $Z$ to muons is suppressed by $\left(1-4 s_{W}^{2}\right) \sim 0.1$ w.r.t. the axial-vector coupling of the $Z$. LFU of $Z$ couplings precludes also the explanation of the violation of this universality in $R_{K}$, hinted at by $\mathrm{LHCb}$ data.

Due to the particular structure of $Z^{\prime}$ couplings, the general pattern of NP contributions to $K^{+} \rightarrow \pi^{+} \nu \bar{\nu}, K_{L} \rightarrow \pi^{0} \nu \bar{\nu}, B_{s, d} \rightarrow \mu \bar{\mu}, B \rightarrow K^{(*)} \mu \bar{\mu}$ and $B \rightarrow K^{(*)} \nu \bar{\nu}$ in $\mathrm{G}_{\mathrm{SM}}^{\prime}(\Phi)$ models is dominated by tree-level $Z$ contributions as in $\mathrm{G}_{\mathrm{SM}}$ models, but because of the aforementioned suppression by $c_{\beta}^{2}$ these contributions are smaller, with few exceptions mentioned above, than in the latter models. On the other hand, the presence of $Z^{\prime}$ with only vector 


\begin{tabular}{|c|c|c|c|c|c|c|c|c|c|c|c|}
\hline & \multicolumn{5}{|c|}{$\mathrm{G}_{\mathrm{SM}}$} & \multicolumn{2}{|c|}{$\mathrm{G}_{\mathrm{SM}}^{\prime}(S)$} & \multicolumn{4}{|c|}{$\mathrm{G}_{\mathrm{SM}}^{\prime}(\Phi)$} \\
\hline & $D$ & $Q_{V}$ & $Q_{d}$ & $T_{d}$ & $T_{u}$ & $D$ & $Q_{V}$ & $D$ & $Q_{d}$ & $T_{d}$ & $T_{u}$ \\
\hline$C_{9}^{i j, \ell}$ & ש & - & - & - & 口 & - & $\star$ & - & - & - & - \\
\hline$C_{9}^{\prime i j, \ell}$ & - & 口 & 口 & - & - & $\star$ & - & - & - & - & - \\
\hline$C_{10}^{i j, \ell}$ & $\star$ & 一 & - & $\star$ & $\star$ & - & - & $\star$ & - & $\star$ & $\star$ \\
\hline$C_{10}^{\prime i j, \ell}$ & - & $\star$ & $\star$ & - & - & - & - & - & $\star$ & - & - \\
\hline$C_{L}^{i j, \nu}$ & $\star$ & - & - & $\star$ & $\star$ & - & $\star$ & $\star$ & - & $\star$ & $\star$ \\
\hline$C_{R}^{i j, \nu}$ & - & $\star$ & $\star$ & - & - & $\star$ & - & - & $\star$ & - & - \\
\hline
\end{tabular}

Table 6. "DNA" table for NP contributions to the $b \rightarrow s \mu^{+} \mu^{-}$Wilson coefficients $C_{9,10}^{(\prime)}$ and to the $d_{j} \rightarrow d_{i} \nu \bar{\nu}$ ones $C_{L, R}^{\nu} \cdot \star$ means that the NP contribution is potentially large, while $\square$ stands for a generically small contribution, due to the suppressed vector couplings of the $Z$ to leptons compared to its axial-vector couplings. Smaller symbols in the $\mathrm{G}_{\mathrm{SM}}^{\prime}(\Phi)$ models indicate the general suppression by $c_{\beta}^{2}$ w.r.t. $\mathrm{G}_{\mathrm{SM}}$ models.

lepton couplings allows in principle to address the $\mathrm{LHCb}$ anomalies more easily; however, given the generic suppression of the $Z^{\prime}$ couplings, this is harder than in $\mathrm{G}_{\mathrm{SM}}^{\prime}(S)$ models.

Hadronic $|\Delta F|=1$ processes governed by $d_{j} \rightarrow d_{i} q \bar{q}$ receive VLQ contributions only from tree-level $Z$ exchange in $\mathrm{G}_{\mathrm{SM}}$ and $\mathrm{G}_{\mathrm{SM}}^{\prime}(\Phi)$ models. The suppression of VLQ effects by $c_{\beta}^{2}$ in $\mathrm{G}_{\mathrm{SM}}^{\prime}(\Phi)$ models w.r.t. $\mathrm{G}_{\mathrm{SM}}$ models is the same as discussed previously for semileptonic $|\Delta F|=1$ processes. Such contributions are entirely absent in $\mathrm{G}_{\mathrm{SM}}^{\prime}(S)$ models and $\varepsilon^{\prime} / \varepsilon$ is generated for example in the case of $d_{j} \rightarrow d_{i} d \bar{d}$ either by $Z^{\prime}$ double insertions or via box diagrams, which are both additionally suppressed by $\left|\lambda_{d}\right|^{2}$ compared to G $\mathrm{G}_{\mathrm{SM}}$ models.

\subsection{Determination of $M$}

There is a common claim that from flavour-violating processes it is only possible to measure the ratio $g_{\mathrm{NP}} / M_{\mathrm{NP}}$, where $g_{\mathrm{NP}}$ is the coupling present in a given theory, while $M_{\mathrm{NP}}$ is the NP scale. The scale tested by a given observable is typically quoted at the value of $M_{\mathrm{NP}}$ when setting $g_{\mathrm{NP}}=1$, and correspondingly changes when the latter is suppressed by some mechanism, as in the case of MFV.

Here we would like to point out that in concrete models with correlations between $|\Delta F|=2$ and $|\Delta F|=1$ processes, it is in general possible to determine $M_{\mathrm{NP}}$ without making any assumptions on the couplings involved. This is in particular important if $M_{\mathrm{NP}}$ should turn out to be beyond the reach of direct searches at the LHC.

In the context of 331 models the relevant correlations that allow the determination of $M_{Z^{\prime}}$ can be found in section 7.2 of [37], although this point has not been made there. In order to illustrate this in the case of VLQ models we consider the $\mathrm{G}_{\mathrm{SM}}$-models. Let us consider the example of $\Delta M_{s}$ and first take into account for the shift $\Delta S$ only box contributions with VLQ exchanges. On the other hand, $\Delta Y$ entering the branching ratio 
for $B_{s} \rightarrow \mu \bar{\mu}$ is governed by tree-level $Z$ exchange. Then we find independently of Yukawa couplings and CKM parameters a useful formula:

$$
\frac{\sqrt{(\Delta S)^{*}}}{\Delta Y}=1.90 b \sqrt{\eta_{m m}}\left[\frac{M}{10 \mathrm{TeV}}\right], \quad \text { (Boxes) }
$$

where $\eta_{m m}$ are given in table 5 and $b=1$ for $D$ and $Q_{V}, b=-1$ for $T_{u}$ and $Q_{d}$ and $b=1 / 2$ for $T_{d}$. Note that $\Delta S$ and $\Delta Y$ are generally complex but their phases are related so that r.h.s. of this equation is real valued. Extracting $\Delta S$ and $\Delta Y$ from experiment, a range for $M$ can be determined.

This formula is modified in the presence of Yukawa RG effects and when the simple tree-level $Z$ contributions cannot be neglected:

- For sufficiently large $M$ the Yukawa RG effects become important. As these contributions have the same dependence on the couplings as $\Delta F=1$ amplitudes and the dependence on the VLQ mass differs only by a logarithm, the determination of $M$ will not be possible if the RG contribution dominates. However, we expect this situation only for RH $\mathrm{G}_{\mathrm{SM}}$ models in the Kaon sector, as explained above. If RG and box contributions are comparable, the determination of $M$ will be possible, although the relevant expressions will be more involved than (5.9).

- For sufficiently low $M$ the tree-level $Z$ contributions to $|\Delta F|=2$ could become important and again dilute the sensitivity to $M$. However, if VLQs are not found at the LHC, the value of $M$ is sufficiently large so that these contributions are numerically irrelevant. On the other hand, if VLQs are discovered at the LHC, we will know their masses and this determination will not be necessary — instead, the determination of the couplings would improve.

In summary the determination of $M$ outside the reach of the LHC will depend on the relevance of box contributions relative to the RG Yukawa effects. Unless RG contributions are clearly dominant, which is only the case in the Kaon sector for $\mathrm{RH}$ scenarios, this determination should be possible by means of a formula like (5.9). The determination is expected to work best for LH scenarios, but also for RH scenarios it should remain possible for $b \rightarrow d, s$ transitions, as discussed in the following section.

\subsection{Kaon and $B$-meson systems}

The correlations between flavour observables in different meson systems are governed by the Yukawa structure of the model in question, as will be elaborated quantitatively in section 6 . The important property of VLQ models is that the products defined in eq. (3.20),

$$
\Lambda_{i j}^{m}=\left|\Lambda_{i j}^{m}\right| e^{i \varphi_{i j}^{m}}
$$

together with the VLQ mass $M$ determine at the same time the flavour-violating $j \rightarrow$ $i$ couplings of $Z$ and $Z^{\prime}$, as well as the flavour-diagonal $Z^{\prime}$ couplings to quarks. The relevant flavour-changing parameters are hence $\Lambda_{d s}^{m}$ in Kaon decays, and $\Lambda_{d b}^{m}, \Lambda_{s b}^{m}$ in $b \rightarrow d, s$ 


\begin{tabular}{|l|c|c|c|c|c|c|c|c|c|c|c|}
\hline & \multicolumn{5}{|c|}{$\mathrm{G}_{\mathrm{SM}}$} & \multicolumn{3}{c|}{$\mathrm{G}_{\mathrm{SM}}^{\prime}(S)$} & \multicolumn{3}{c|}{$\mathrm{G}_{\mathrm{SM}}^{\prime}(\Phi)$} \\
& $D$ & $Q_{V}$ & $Q_{d}$ & $T_{d}$ & $T_{u}$ & $D$ & $Q_{V}$ & $D$ & $Q_{d}$ & $T_{d}$ & $T_{u}$ \\
\hline$|\Delta F|=2$ & $\star$ & $\star$ & $\star$ & $\star$ & $\star$ & $\star$ & $\star$ & $\star$ & $\star$ & $\star$ & $\star$ \\
\hline$B_{s, d} \rightarrow \mu \bar{\mu}$ & $\star$ & $\star$ & $\star$ & $\star$ & $\star$ & & & $\star$ & $\star$ & $\star$ & $\star$ \\
\hline$B \rightarrow K \mu \bar{\mu}$ & $\star$ & $\star$ & $\star$ & $\star$ & $\star$ & $\star$ & $\star$ & $\star$ & $\star$ & $\star$ & $\star$ \\
\hline$B \rightarrow K^{*} \mu \bar{\mu}$ & & & & & & $\star$ & $\star$ & $\star$ & $\star$ & $\star$ & $\star$ \\
\hline$B \rightarrow K\left(K^{*}\right) \nu \bar{\nu}$ & $\star$ & $\star$ & $\star$ & $\star$ & $\star$ & & & $\star$ & $\star$ & $\star$ & $\star$ \\
\hline$K^{+} \rightarrow \pi^{+} \nu \bar{\nu}$ & $\star$ & $\star$ & $\star$ & $\star$ & $\star$ & & & $\star$ & $\star$ & $\star$ & $\star$ \\
\hline$K_{L} \rightarrow \pi^{0} \nu \bar{\nu}$ & $\star$ & $\star$ & $\star$ & $\star$ & $\star$ & & & & & & \\
\hline$\varepsilon^{\prime} / \varepsilon$ & $\star$ & $\star$ & $\star$ & $\star$ & $\star$ & & & $\star$ & $\star$ & $\star$ & $\star$ \\
\hline
\end{tabular}

Table 7. "DNA" of flavour effects in VLQ models. A star indicates that significant effects in a given model and given process are in principle possible, but could be reduced (see section 6) through correlation among several observables. Empty space means that the given model does not predict sizeable effects in that observable. The star $\star$ indicates left-handed currents and the star $\star$ right-handed ones, smaller stars indicate the suppression of $|\Delta F|=1$ decays in $\mathrm{G}_{\mathrm{SM}}^{\prime}(\Phi)$ models.

transitions of $B$ mesons, respectively. Since only the relative phases of the $\lambda_{i}^{\mathrm{VLQ}}$ enter the $\Lambda_{i j}^{m}$, the phases $\varphi_{i j}^{m}$ fulfill the relation

$$
\varphi_{b s}=\varphi_{b d}-\varphi_{s d},
$$

dropping the index $m$ of the VLQ representation for convenience. This leaves us with five parameters for the three complex quantities $\Lambda_{i j}$. The phases $\varphi_{i j}$ can vary in the full range $[-\pi, \pi]$, implying the occurrence of discrete ambiguities when determining them from experiment, as explicitly seen in the plots in ref. [35] and in the plots in the next section. They can be resolved using observables where interference with the SM occurs. The absolute values $\lambda_{i}^{\mathrm{VLQ}}$ can be determined via

$$
\left|\lambda_{d}\right|=\sqrt{\frac{\Lambda_{b d} \Lambda_{s d}^{*}}{\Lambda_{b s}}}, \quad\left|\lambda_{s}\right|=\sqrt{\frac{\Lambda_{b s} \Lambda_{s d}}{\Lambda_{b d}}}, \quad\left|\lambda_{b}\right|=\sqrt{\frac{\Lambda_{b d} \Lambda_{b s}^{*}}{\Lambda_{s d}}} .
$$

One might expect the strongest constraints numerically to stem from $s \rightarrow d$ processes, because of the strong suppression of the SM contribution by $V_{t d} V_{t s}^{*}$.

In a sense, as more explicitly seen in the next section, the flavour structure of VLQ models has some parallels to the one in 331 models $[37,56,57,65]$. However, in 331 models the NP contributions are dominated by $Z^{\prime}$ tree-level exchanges and once the constraints from $B_{s, d}$ observables are taken into account, NP effects in the $K$ system are found to be small, with the exception of $\varepsilon^{\prime} / \varepsilon$. In the present analysis important $Z$ 
boson contributions are present and this allows for more interesting NP effects than in 331 models in $K^{+} \rightarrow \pi^{+} \nu \bar{\nu}$ and $K_{L} \rightarrow \pi^{0} \nu \bar{\nu}$. Furthermore, the partial decoupling of $|\Delta F|=1$ and $|\Delta F|=2$ processes due to the presence of important box diagram contributions to $|\Delta F|=2$ processes in VLQ models discussed above modifies the corresponding correlations derived in ref. [35], increasing the impact of $|\Delta F|=2$ constraints on $|\Delta F|=1$ processes relative to the one found in [35]. The latter is also true for RG effects in $\mathrm{G}_{\mathrm{SM}}$ models, specifically for $\mathrm{RH}$ scenarios, where the importance of $\Delta F=2$ can be drastically enhanced. In table 7 we summarize the patterns discussed above.

\section{Numerics}

In this section we perform the numerical analysis of the VLQ models presented above. For this purpose we start by constraining the VLQ couplings by the available flavour data and if applicable also by data from other sectors. We proceed by presenting the predictions for a number of key observables given these constraints, including their correlations where they are sizeable. These fits are performed for different VLQ masses, in order to illustrate the explicit mass dependence of flavour observables discussed in section 5.3.

Model-independent constraints on $\psi^{2} \varphi^{2} D$ operators have been derived from $Z$ - and $W$ boson observables [66], which are applicable to $\mathrm{G}_{\mathrm{SM}}$ models. Although these constraints are not entirely independent from other operators, in VLQ-models the latter are loopsuppressed and can be neglected. The constraints on the modulus of the couplings are weak and of the order $\left|\lambda_{i}\right| \lesssim M /(1 \mathrm{TeV}) .{ }^{14}$

More stringent constraints derive from $|\Delta F|=2,1$ flavour observables [11]. We constrain the five parameters $\left|\Lambda_{i j}\right|$ and $\varphi_{i j}$ (5.10) with the $|\Delta F|=2,1$ processes listed in table 8. Master formulae used in these constraints are collected in appendix C. The SM predictions in table 8 are based on the determination of CKM parameters from a tree-level fit given in table 13. Some comments regarding the included observables are in order:

- The observable $\Delta M_{K}$ does not provide constraints in $\mathrm{G}_{\mathrm{SM}}$ models and is omitted due to too large uncertainties from long-distance contributions in $\mathrm{G}_{\mathrm{SM}}^{\prime}(\Phi)$ models. The prospects for controlling this long-distance part by lattice calculations are good [67] and in the future this constraint could play an important role.

- We find that huge NP effects in $\varepsilon^{\prime} / \varepsilon$ are not excluded by the constraints listed in table 8 in $\mathrm{G}_{\mathrm{SM}^{-}}$and $\mathrm{G}_{\mathrm{SM}}^{\prime}(\Phi)$-models, such that we impose bounds on the NP contribution $\left(\varepsilon^{\prime} / \varepsilon\right)_{\mathrm{NP}}$ itself

$$
\left(\varepsilon^{\prime} / \varepsilon\right)_{\mathrm{NP}} \in[0,20] \times 10^{-4},
$$

in order to avoid showing predictions for other observables that are easily excluded by $\varepsilon^{\prime} / \varepsilon$, and to analyse its influence on the correlations of observables. This range roughly corresponds to NP required assuming present predictions from lattice QCD. We have checked that decreasing this range to $[5,10] \times 10^{-4}$ as expected from the

\footnotetext{
${ }^{14}$ There is one tension from $\left[\hat{c}_{H d}\right]_{33}=(-4.6 \pm 1.6) \times 10^{-2}[66]$ (A.9) for the VLQ representation $Q_{V}$.
} 


\begin{tabular}{|c|c|c|c|c|}
\hline$i \rightarrow j$ & observable & measurement & ref. & SM (c.v. $[95 \%$ CL] $)$ \\
\hline \multirow{4}{*}{$s \rightarrow d$} & $\varepsilon_{K}$ & $2.228(11) \times 10^{-3}$ & {$[72]$} & $(2.21[1.57,2.98]) \times 10^{-3}$ \\
\hline & $\operatorname{Br}\left(K^{+} \rightarrow \pi^{+} \nu \bar{\nu}\right)$ & $\left(17.3_{-10.5}^{+11.5}\right) \times 10^{-11}$ & {$[73]$} & $(8.5[7.3,9.5]) \times 10^{-11}$ \\
\hline & $B r\left(K_{L} \rightarrow \mu \bar{\mu}\right)_{\mathrm{SD}}$ & $<2.5 \times 10^{-9}$ & {$[74]$} & $\chi_{\mathrm{SD}}: 1.81[1.65,1.94]$ \\
\hline & $\left(\varepsilon^{\prime} / \varepsilon\right)_{\mathrm{NP}}$ & {$[0,20] \times 10^{-4}$} & $\dagger$ & $0 \times 10^{-4}$ \\
\hline \multirow{3}{*}{$b \rightarrow d$} & $\Delta M_{d}\left[\mathrm{ps}^{-1}\right]$ & $0.5055(20)$ & {$[75]$} & $0.62[0.45,0.78]$ \\
\hline & $\sin \left(2 \beta_{d}\right)$ & $0.691(17)^{*}$ & {$[75]$} & $0.734[0.686,0.796]$ \\
\hline & $\operatorname{Br}\left(B^{+} \rightarrow \pi^{+} \mu \bar{\mu}\right)_{[15,22]}$ & $3.29(84) \times 10^{-9}$ & {$[76]$} & $(5.0[3.8,7.2]) \times 10^{-9}$ \\
\hline \multirow{4}{*}{$b \rightarrow s$} & $\Delta M_{s}\left[\mathrm{ps}^{-1}\right]$ & $17.757(21)$ & {$[75]$} & $19.0[16.2,21.9]$ \\
\hline & $\sin \left(2 \beta_{s}\right)$ & $-0.034(33)^{*}$ & {$[75]$} & $-0.040[-0.044,-0.036]$ \\
\hline & $\operatorname{Br}\left(B_{s} \rightarrow \mu \bar{\mu}\right)$ & $\left(2.8_{-0.6}^{+0.7}\right) \times 10^{-9}$ & {$[77]$} & $(3.41[3.01,3.81]) \times 10^{-9}$ \\
\hline & $\operatorname{Br}\left(B^{+} \rightarrow K^{+} \mu \bar{\mu}\right)_{[15,22]}$ & $8.47(50) \times 10^{-8}$ & {$[78]$} & $(11.0[6.4,15.6]) \times 10^{-8}$ \\
\hline
\end{tabular}

Table 8. The list of $|\Delta F|=2,1$ flavour observables in $i \rightarrow j$ down-type transitions that are used to constrain the VLQ couplings. SM predictions are obtained with CKM parameters determined from the tree-fit. ${ }^{\dagger}$ We impose this conservative range on the NP contribution of $\varepsilon^{\prime} / \varepsilon$ to avoid values excluded by this observable in the predictions for other observables, see text for more details. * Note that we neglect potential "penguin pollution" in $b \rightarrow c \bar{c} s$ transitions, which have been shown in recent analyses to be at most of the size of the present experimental uncertainties [79-81].

dual approach to QCD [21, 45] would have only minor impact on the global fit as what matters is the unique selection of the sign of the relevant phase required for the enhancement of $\varepsilon^{\prime} / \varepsilon$.

- Due to the sizeable experimental uncertainties, $\operatorname{Br}\left(B_{d} \rightarrow \mu \bar{\mu}\right)$ does not constrain the VLQ parameters further. It is thus omitted from the fit and we compare its prediction in our models to the present measurement.

- A full analysis of $B \rightarrow K^{*} \ell \bar{\ell}$ is beyond the scope of this work. We do therefore not include the LHCb anomalies [68-71] in our fits. The analysis of $b \rightarrow s \ell \bar{\ell}$ in $\mathrm{G}_{\mathrm{SM}}^{\prime}(S)$ models has been already presented in $[9,15]$ and we have nothing to add here. In $\mathrm{G}_{\mathrm{SM}}$ models the shift in $C_{9}$ is too small to be relevant, while in $\mathrm{G}_{\mathrm{SM}}^{\prime}(\Phi)$ models the effects are only moderately interesting and we will not address them here.

The three sectors $s \rightarrow d, b \rightarrow d$ and $b \rightarrow s$ are not independent, due to relation (5.11). In our analysis we show first the results separately for the three quark transitions and demonstrate in a global fit that $K$-physics constraints have an impact on $B$ physics but not vice versa. 


\section{1 $\quad \mathrm{G}_{\mathrm{SM}}$ models}

In $\mathrm{G}_{\mathrm{SM}}$ models the absence of additional scalars allows to vary the mass of the VLQ's down to about $1 \mathrm{TeV}$ without violating the hierarchy (3.1). The fits of the $\Lambda_{i j}$ for the three types of transitions $j \rightarrow i=\{s \rightarrow d, b \rightarrow d, b \rightarrow s\}$ in $\mathrm{G}_{\mathrm{SM}}$ models are shown in figure 5 for $M_{\mathrm{VLQ}}=10 \mathrm{TeV}$ and in figure 6 for $M_{\mathrm{VLQ}}=1 \mathrm{TeV}$ for the single-VLQ scenarios $D$ and $Q_{V}$ with LH and RH couplings, respectively. The plots for LH scenarios $T_{u, d}$ are qualitatively similar to $D$ whereas the $\mathrm{RH}$ scenario $Q_{d}$ is similar to $Q_{V}$. Quantitative differences arise due to changes of the sign in couplings and a factor $1 / 2$ for $T_{d}$ w.r.t. $D$ and $T_{u}$, which are shown in table 3. The statistical approach for these fits is detailed in appendix D. We make the following observations:

- All included observables are compatible with the SM prediction at 95\% CL. Correspondingly also the global fit allows for the SM solution at 95\% CL in all planes in both scenarios, except for $\Lambda_{b d}^{Q_{V}}$ with $M_{\mathrm{VLQ}}^{Q_{V}}=1,10 \mathrm{TeV}$, where the SM is slightly outside that region. This is due to the slight tensions of $\Delta M_{d}$ and $\operatorname{Br}\left(B^{+} \rightarrow \pi^{+} \ell \bar{\ell}\right)$ with their SM predictions, which fortify each other in this case.

- For $M_{\mathrm{VLQ}}=10 \mathrm{TeV},|\Delta F|=2$ constraints are competitive to the $|\Delta F|=1$ ones, and their interplay determines the global fit regions. For $M_{\mathrm{VLQ}}=1 \mathrm{TeV}$ the global fit is almost completely determined by $|\Delta F|=1$ processes in LH scenarios, but also in $\mathrm{RH}$ scenarios for $b \rightarrow d, s$. On the other hand, $\epsilon_{K}$ is a very powerful constraint in $\mathrm{RH}$ scenarios also for $1 \mathrm{TeV}$, due to the RG effects discussed above. This is in accordance with our previous discussion of the mass-dependence of these transitions. Specifically for $K^{+} \rightarrow \pi^{+} \nu \bar{\nu}$, large effects are excluded by $\epsilon_{K}$ in combination with $\varepsilon^{\prime} / \varepsilon$ and $K_{L} \rightarrow \mu \bar{\mu}$. Without the RG contributions, enhancements up to the present experimental limit would have been possible.

- In $b \rightarrow s$, the $|\Delta F|=1$ observables distinguish between scenarios with LH and RH currents due to their different dependences on the corresponding Wilson coefficients, most importantly $C_{10}$ and $C_{10}^{\prime}$,

$$
B r\left(B_{s} \rightarrow \mu \bar{\mu}\right) \propto\left|C_{10}-C_{10}^{\prime}\right|^{2}, \quad B r\left(B^{+} \rightarrow K^{+} \mu \bar{\mu}\right) \propto\left|C_{10}+C_{10}^{\prime}\right|^{2} .
$$

The consequence is shown in figure 5 and figure 6 where allowed regions almost overlap for LH scenarios, but intersect only around the SM for RH scenarios, thereby diminishing the size of potential VLQ effects in other $b \rightarrow s$ observables. The same observation holds for $b \rightarrow d$ transitions, which will help once $B_{d} \rightarrow \mu \bar{\mu}$ is measured more precisely. In figure 11 we illustrate how $\operatorname{Br}\left(B_{d, s} \rightarrow \mu \bar{\mu}\right)$ can be used in a large region of parameter space to discriminate between $\mathrm{LH}$ and $\mathrm{RH}$ models.

- In $s \rightarrow d$ transitions, the constraints from $\epsilon_{K},\left(\varepsilon^{\prime} / \varepsilon\right)_{\mathrm{NP}}$ and $\operatorname{Br}\left(K_{L} \rightarrow \mu \bar{\mu}\right)_{\mathrm{SD}}$ constrain the allowed values for $\varphi_{s d}$. This in combination with the slight tensions especially in $b \rightarrow d$ leads to stronger constraints in the global fit compared to the fits for the individual transitions in $b \rightarrow d, s$. As a consequence correlations between different 

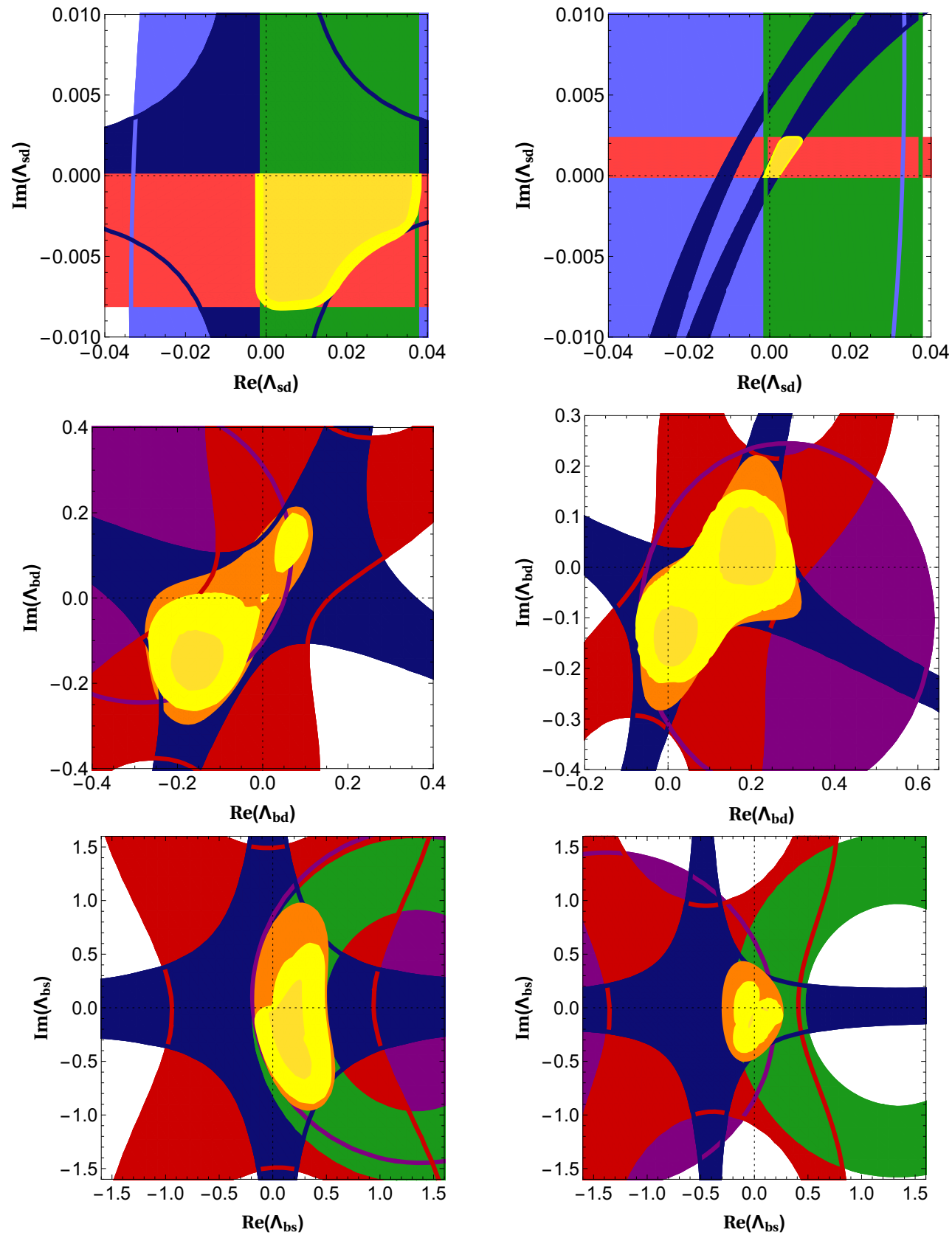

Figure 5. Fits of $\operatorname{Im}\left(\Lambda_{i j}\right)$ vs. $\operatorname{Re}\left(\Lambda_{i j}\right)$ for $i j=s d, b d$, bs [upper, middle, lower] in $\mathrm{G}_{\mathrm{SM}}$-scenarios $D$ [left] and $Q_{V}$ [right] for $M_{\mathrm{VLQ}}=10 \mathrm{TeV}$. Constraints from single observables and the combined fit for each separate sector [orange] are shown at $95 \% \mathrm{CL}$, the global fit [yellow] at $68 \%$ and $95 \%$. For $i j=s d$ : $\varepsilon_{K}$ [dark blue], $B r\left(K^{+} \rightarrow \pi^{+} \nu \bar{\nu}\right)$ [blue], $B r\left(K_{L} \rightarrow \mu \bar{\mu}\right)_{\mathrm{SD}}$ [green], and $\left(\varepsilon^{\prime} / \varepsilon\right)_{\mathrm{NP}}$ [red]. For $i j=b d: \Delta M_{d}$ [dark red], $\sin \left(2 \beta_{d}\right)$ [dark blue] and $B r\left(B^{+} \rightarrow \pi^{+} \mu \bar{\mu}\right)_{[15,22]}$ [purple]. For $i j=b s$ : $\Delta M_{s}$ [dark red], $\sin \left(2 \beta_{s}\right)$ [dark blue], $\operatorname{Br}\left(B_{s} \rightarrow \mu \bar{\mu}\right)$ [green] and $\operatorname{Br}\left(B^{+} \rightarrow K^{+} \mu \bar{\mu}\right)_{[15,22]}$ [purple]. 

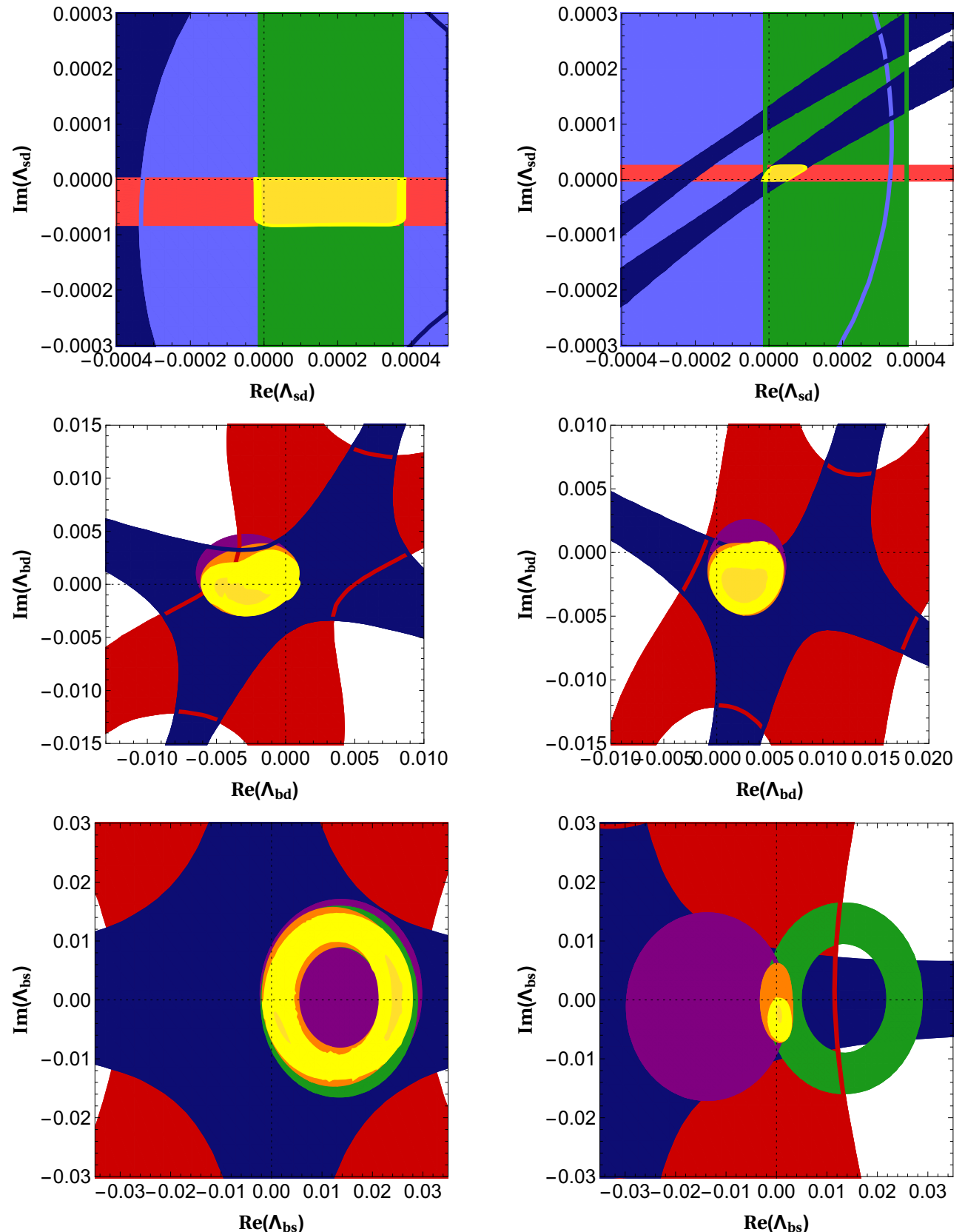

Figure 6. Fits of $\operatorname{Im}\left(\Lambda_{i j}\right)$ vs. $\operatorname{Re}\left(\Lambda_{i j}\right)$ for $i j=s d, b d$, sd [upper, middle, lower] in $\mathrm{G}_{\mathrm{SM}}$-scenarios $D$ [left] and $Q_{V}$ [right] for $M_{\mathrm{VLQ}}=1 \mathrm{TeV}$. The colour scheme is as in figure 5 . 
transitions arise, but at the moment they are not very strong yet. This would change with significant measurements away from the SM for at least two of the transitions.

- The $|\Delta F|=2$ CP-asymmetric observables $\epsilon_{K}$ and $\sin \left(2 \beta_{d, s}\right)$ impose constraints in the complex $\Lambda_{i j}$-planes, which are not limited along the direction corresponding to the SM phase. Such a limit is provided by $\Delta M_{d, s}$, whereas in the case of $s \rightarrow d$ the one from $\Delta M_{K}$ is very weak and outside of the ranges shown.

- There is a complementarity in the constraints from $\operatorname{Br}\left(K^{+} \rightarrow \pi^{+} \nu \bar{\nu}\right)$ and $\operatorname{Br}\left(K_{L} \rightarrow\right.$ $\mu \bar{\mu})_{\mathrm{SD}}$ for every VLQ representation. Thus an improved measurement of $\operatorname{Br}\left(K^{+} \rightarrow\right.$ $\left.\pi^{+} \nu \bar{\nu}\right)$ by NA62, which will operate until the LHC shut down in 2018 and aims at a $10 \%$ uncertainty $[82,83]$, will provide stronger cuts into the allowed parameter space. On the other hand, while the constraints from $\left(\varepsilon^{\prime} / \varepsilon\right)_{\mathrm{NP}}$ and $\operatorname{Br}\left(K_{L} \rightarrow \mu \bar{\mu}\right)_{\mathrm{SD}}$ are theoretically limited at present, they could become very powerful in the future if theory improves.

Using the above constraints, we obtain allowed ranges for observables that are yet to be measured (precisely), listed in table 9. We furthermore analyze patterns for each transition, that will help to distinguish VLQ models from other NP scenarios, and different VLQs from each other. In this respect we point out that models $Q_{d}$ and $Q_{V}$ have the same experimental signatures in down-type quark FCNC transitions and are hence indistinguishable. Such a distinction might be possible after invoking additional constraints from up-type quark FCNC transitions, where both models differ from each other as indicated in eq. (2.5). Still, in $Q_{V}$ models strong correlations between the up- and down-type sectors are not expected due to the in principle independent up- and down-type Yukawa couplings.

In the Kaon sector, we make the following observations, see also figure 7:

- The VLQ models allow to enhance $\varepsilon^{\prime} / \varepsilon$ significantly, thereby addressing the apparent gap between the SM prediction and data, at the expense of suppressing $\operatorname{Br}\left(K_{L} \rightarrow\right.$ $\left.\pi^{0} \nu \bar{\nu}\right)$. This suppression is significantly weaker for $Q_{V}$ and $Q_{d}$ models (RH currents) than for $D, T_{d}$ and $T_{u}$ (LH currents), in accordance with the general study in [40]. Simultaneous agreement with the data for $\varepsilon_{K}$ and $\varepsilon^{\prime} / \varepsilon$ can be obtained without fine-tuning of parameters.

- While the impact of $\varepsilon^{\prime} / \varepsilon$ on $K_{L} \rightarrow \pi^{0} \nu \bar{\nu}$ is large as stated above, $K^{+} \rightarrow \pi^{+} \nu \bar{\nu}$ and $\varepsilon^{\prime} / \varepsilon$ are only weakly correlated. However, in RH models $\epsilon_{K}$ prevents large enhancements of $\operatorname{Br}\left(K^{+} \rightarrow \pi^{+} \nu \bar{\nu}\right)$, the maximal enhancement is about $50 \%$ of its SM value. In models with LH currents, a strong suppression is possible, and the SM value corresponds to an upper bound in this case when a stricter bound from $K_{L} \rightarrow \mu \bar{\mu}$ is used. This implies that a measurement of a significantly enhanced $\operatorname{Br}\left(K^{+} \rightarrow \pi^{+} \nu \bar{\nu}\right)$, as presently still allowed by data, could exclude all $\mathrm{G}_{\mathrm{SM}}$ models with a single VLQ representation, although in models with LH currents a more conservative bound from $K_{L} \rightarrow \mu \bar{\mu}$ would presently still allow the enhancement of $\operatorname{Br}\left(K^{+} \rightarrow \pi^{+} \nu \bar{\nu}\right)$ up to a factor of two.

- In this context it should be again emphasized that the modes $K^{+} \rightarrow \pi^{+} \nu \bar{\nu}$ and $K_{L} \rightarrow \mu \bar{\mu}$ are strongly correlated in VLQ models, however, again differently so for 


\begin{tabular}{|c|c|c|c|c|c|}
\hline $\mathrm{SM}$ & measurement & $D$ & $Q_{d}, Q_{V}$ & $T_{u}$ & $T_{d}$ \\
\hline \multicolumn{6}{|c|}{$10^{11} \times B r\left(K_{L} \rightarrow \pi^{0} \nu \bar{\nu}\right), \quad 10^{11} \times B r\left(K^{+} \rightarrow \pi^{+} \nu \bar{\nu}\right)$} \\
\hline $3.2[2.5,4.3]$ & $\leq 2600 \quad[84]$ & $\begin{array}{l}{[0,4.3]} \\
{[0,4.3]}\end{array}$ & $\begin{array}{l}{[1.3,3.3]} \\
{[1.2,3.5]}\end{array}$ & $\begin{array}{l}{[0,4.3]} \\
{[0,4.3]}\end{array}$ & $\begin{array}{l}{[0,4.2]} \\
{[0,4.2]}\end{array}$ \\
\hline $8.5[7.3,9.5]$ & $17.3_{-10.5}^{+11.5} \quad[84]$ & $\begin{array}{l}{[0.8,9.2]} \\
{[0.8,9.2]} \\
\end{array}$ & $\begin{array}{l}{[7.7,13.6]} \\
{[7.8,13.0]}\end{array}$ & $\begin{array}{l}{[0.7,8.9]} \\
{[0.8,9.2]}\end{array}$ & $\begin{array}{l}{[0.7,8.9]} \\
{[1.2,9.2]} \\
\end{array}$ \\
\hline \multicolumn{6}{|c|}{$10^{10} \times B r\left(B_{d} \rightarrow \mu \bar{\mu}\right)$} \\
\hline $1.14[0.94,1.32]$ & $\leq 6.3 \quad[85]$ & $\begin{array}{l}{[0.0,1.7]} \\
{[0.1,1.8]}\end{array}$ & $\begin{array}{l}{[1.2,10.4]} \\
{[1.0,4.0]}\end{array}$ & $\begin{array}{l}{[0.0,1.7]} \\
{[0.1,1.9]} \\
\end{array}$ & $\begin{array}{l}{[0.0,1.7]} \\
{[0.5,1.7]}\end{array}$ \\
\hline \multicolumn{6}{|c|}{$A_{\Delta \Gamma}\left(B_{s} \rightarrow \mu \bar{\mu}\right), \quad S\left(B_{s} \rightarrow \mu \bar{\mu}\right)$} \\
\hline 1 & - & $\begin{array}{l}{[-1.00,1.00]} \\
{[0.12,0.99]}\end{array}$ & $\begin{array}{l}{[0.67,1.00]} \\
{[0.87,1.00]}\end{array}$ & $\begin{array}{l}{[-1.00,1.00]} \\
{[0.46,1.00]}\end{array}$ & $\begin{array}{l}{[-0.28,1.00]} \\
{[0.86,1.00]}\end{array}$ \\
\hline 0 & - & $\begin{array}{l}{[-1.00,1.00]} \\
{[-0.99,0.99]}\end{array}$ & $\begin{array}{l}{[-0.63,0.74]} \\
{[-0.41,0.48]}\end{array}$ & $\begin{array}{l}{[-1.00,1.00]} \\
{[-0.87,0.89]}\end{array}$ & $\begin{array}{l}{[-1.00,1.00]} \\
{[-0.49,0.51]}\end{array}$ \\
\hline \multicolumn{6}{|c|}{$10^{2} \times A_{7,8,9}\left(B \rightarrow K^{*} \mu \bar{\mu}\right)_{[1,6]}$} \\
\hline$<0.1$ & $4.5 \pm 5.0 \quad[86]$ & $\begin{array}{l}{[-23.4,23.3]} \\
{[-14.5,14.1]}\end{array}$ & $\begin{array}{l}{[-8.9,7.4]} \\
{[-5.9,5.0]}\end{array}$ & $\begin{array}{l}{[-23.7,23.7]} \\
{[-12.0,11.9]}\end{array}$ & $\begin{array}{l}{[-18.3,17.3]} \\
{[-6.1,5.8]}\end{array}$ \\
\hline$<0.1$ & $-4.7 \pm 5.8 \quad[86]$ & $\begin{array}{l}{[-0.9,0.9]} \\
{[-0.5,0.5]}\end{array}$ & $\begin{array}{l}{[-6.9,5.8]} \\
{[-4.6,3.9]}\end{array}$ & $\begin{array}{l}{[-0.9,0.8]} \\
{[-0.4,0.4]}\end{array}$ & $\begin{array}{l}{[-0.6,0.6]} \\
{[-0.2,0.2]}\end{array}$ \\
\hline$<0.1$ & $3.3 \pm 4.2 \quad[86]$ & $\mathrm{SM}$ & $\begin{array}{l}{[-3.5,4.2]} \\
{[-2.4,2.8]} \\
\end{array}$ & $\mathrm{SM}$ & SM \\
\hline \multicolumn{6}{|c|}{$10^{2} \times A_{8,9}\left(B \rightarrow K^{*} \mu \bar{\mu}\right)_{[15,19]}$} \\
\hline$<0.1$ & $2.5 \pm 4.8 \quad[86]$ & $\mathrm{SM}$ & $\begin{array}{l}{[-5.2,4.3]} \\
{[-3.5,2.9]}\end{array}$ & $\mathrm{SM}$ & $\mathrm{SM}$ \\
\hline$<0.1$ & $-6.1 \pm 4.3[86]$ & $\mathrm{SM}$ & $\begin{array}{l}{[-7.8,9.4]} \\
{[-5.2,6.3]}\end{array}$ & $\mathrm{SM}$ & $\mathrm{SM}$ \\
\hline \multicolumn{6}{|c|}{$\mathcal{R}_{B \rightarrow K \nu \bar{\nu}}, \quad \mathcal{R}_{B \rightarrow K^{*} \nu \bar{\nu}}, \quad \mathcal{R}_{F_{L}}$} \\
\hline 1 & $\leq 4.3$ & $\begin{array}{l}{[0.02,1.10]} \\
{[0.63,1.10]}\end{array}$ & $\begin{array}{l}{[0.79,1.24]} \\
{[0.78,1.20]}\end{array}$ & $\begin{array}{l}{[0.01,1.10]} \\
{[0.65,1.11]}\end{array}$ & $\begin{array}{l}{[0.62,1.11]} \\
{[0.71,1.12]}\end{array}$ \\
\hline 1 & $\leq 4.4$ & $\begin{array}{l}{[0.02,1.10]} \\
{[0.63,1.10]}\end{array}$ & $\begin{array}{l}{[0.87,1.17]} \\
{[0.88,1.17]}\end{array}$ & $\begin{array}{l}{[0.01,1.10]} \\
{[0.65,1.11]}\end{array}$ & $\begin{array}{l}{[0.62,1.11]} \\
{[0.71,1.12]}\end{array}$ \\
\hline 1 & - & $\mathrm{SM}$ & $\begin{array}{l}{[0.92,1.07]} \\
{[0.93,1.07]}\end{array}$ & $\mathrm{SM}$ & $\mathrm{SM}$ \\
\hline
\end{tabular}

Table 9. Ranges still allowed for observables when taking the constraints from table 8 for the individual $s \rightarrow d, b \rightarrow d$ and $b \rightarrow s$ sectors into account, fitting at the time same CKM and hadronic parameters. Upper and lower intervals are for $M_{\mathrm{VLQ}}=1 \mathrm{TeV}$ and $10 \mathrm{TeV}$, respectively. Entries denoted as "SM" have tiny or no deviations from the SM. Experimental upper bounds are given at $90 \% \mathrm{CL}$. 

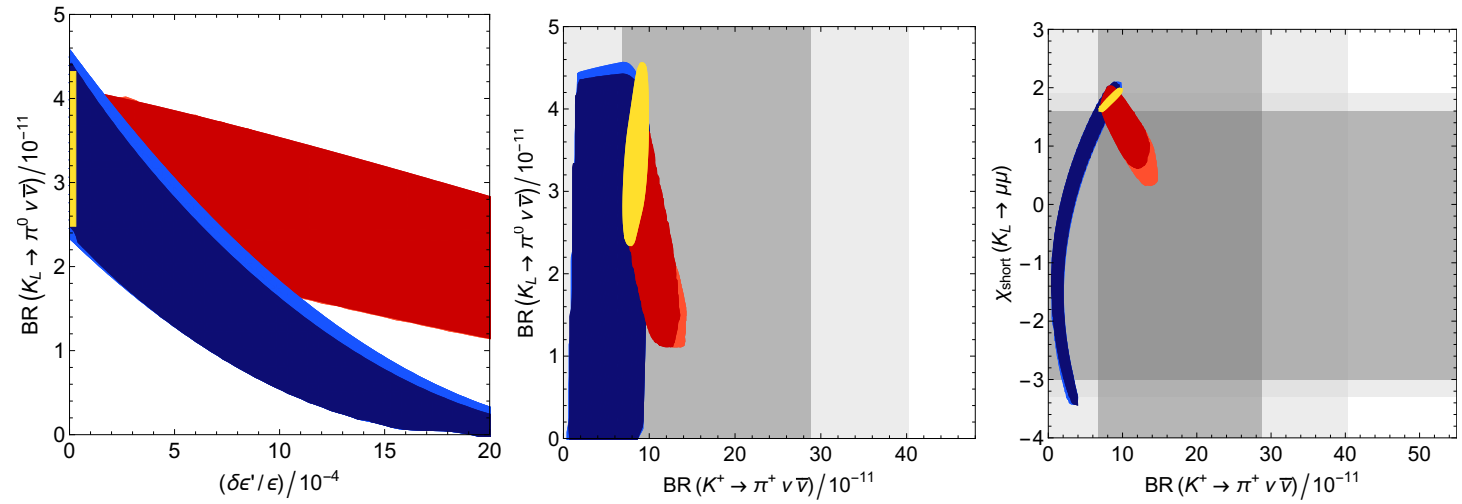

Figure 7. The correlations of observables in the Kaon sector in $\mathrm{G}_{\mathrm{SM}}$ scenarios at $95 \%$ CL for the SM, as well as for $M_{\mathrm{VLQ}}=10 \mathrm{TeV}$ [darker colours] and $M_{\mathrm{VLQ}}=1 \mathrm{TeV}$ [lighter colours]. The colours correspond to the SM prediction [Yellow] and the VLQ-representations $D$ [Blue] and $Q_{V}$ [Red]; the results for $T_{u, d}$ and $Q_{d}$ are very similar to the former and the latter, respectively. Dark and light grey bands show experimental measurements at 1- and $2 \sigma$.

$\mathrm{LH}$ and $\mathrm{RH}$ currents. While for RH currents one can easily infer the allowed range in one mode from a determination of the other, within the limited range allowed by $\varepsilon^{\prime} / \varepsilon$ and $\epsilon_{K}$, LH-current models are more strongly constrained from $K_{L} \rightarrow \mu \bar{\mu}$. Progress for the latter mode depends solely on the capability to separate the long-distance contributions to this mode from the short-distance ones, since the relevant data are already very precise, see appendix C. Note that there is basically no correlation between $\varepsilon^{\prime} / \varepsilon$ and $K_{L} \rightarrow \mu \bar{\mu}$, as they are governed by imaginary and real parts of the corresponding couplings, respectively.

- The VLQ mass does not have a large impact on all these correlations, as can be seen by comparing the lighter and darker areas in figure 7 . The reason is in LH models that $|\Delta F|=1$ transitions are the dominant constraints at both masses, rendering the allowed ranges for other $|\Delta F|=1$ processes mass-independent. For RH models, the same conclusion is reached by considering additionally the fact that $\epsilon_{K}$ is dominated by RG-induced contributions which scale similarly to $|\Delta F|=1$ ones.

Correlation plots for observables in $b \rightarrow s$ processes are shown in figure 8 . We observe the following patterns:

- Since NP effects in all three quark transitions are governed by different parameters, the slight tensions in $|\Delta F|=2$ observables hinted at by new lattice data [61] can easily be removed in VLQ models. This is in contrast to constrained-MFV models, where $\varepsilon_{K}$ prohibits large effects in $\Delta M_{d, s}[62]$.

- $\operatorname{Br}\left(B_{s} \rightarrow \mu \bar{\mu}\right)$ can be strongly suppressed below its SM value, as slightly favoured by experiment, while still allowing for sizeable NP effects in $\sin \left(2 \beta_{s}\right)$, in particular in the case of models with $\mathrm{LH}$ currents. For $M_{\mathrm{VLQ}}=1 \mathrm{TeV}|\Delta F|=1$ observables constrain the NP effects in $\phi_{s}$ to be smaller than for larger VLQ masses. 

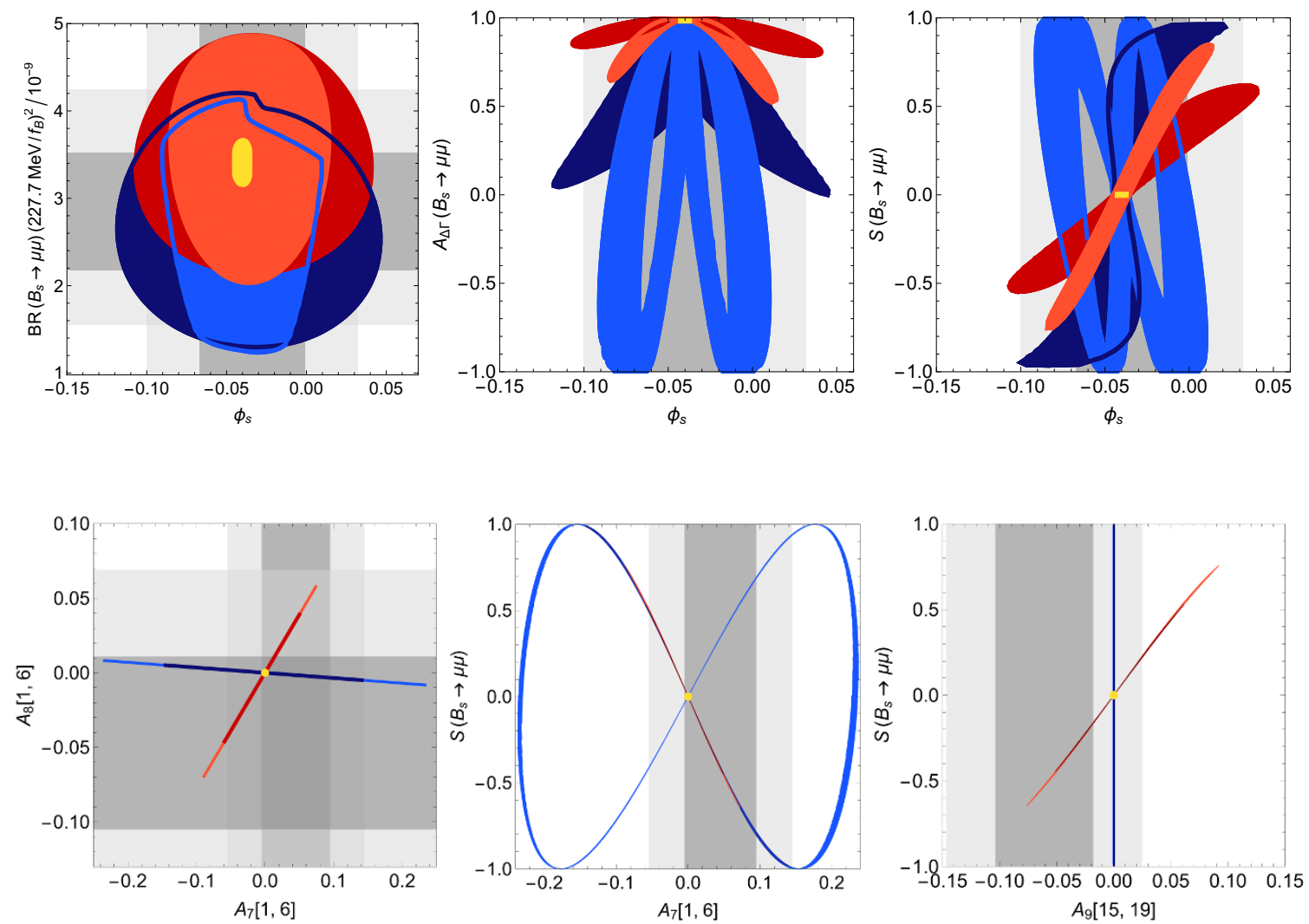

Figure 8. The correlations of observables in the $b \rightarrow s$ sector in $\mathrm{G}_{\mathrm{SM}}$-scenarios for $M_{\mathrm{VLQ}}=$ $10 \mathrm{TeV}$ [darker colours] and $1 \mathrm{TeV}$ [lighter colours] within the $95 \% \mathrm{CL}$ regions. The colours are for VLQ-representations $D$ [Blue] (similar to $T_{u, d}$ ), $Q_{V}=Q_{d}$ [Red]. Grey bands show experimental measurements at 1- and $2 \sigma$ and the yellow dots are the SM predictions.

- Sizeable deviations from the SM prediction are still possible for the mass-eigenstate rate asymmetry $A_{\Delta \Gamma}\left(B_{s} \rightarrow \mu \bar{\mu}\right)$ and the mixing-induced CP-asymmetry $S\left(B_{s} \rightarrow\right.$ $\mu \bar{\mu})$. Indeed, both can essentially vary in the full range $[-1,1]$ for LH models for $M_{\mathrm{VLQ}}=1 \mathrm{TeV}$. For RH models, $A_{\Delta \Gamma}\left(B_{s} \rightarrow \mu \bar{\mu}\right) \geq 50 \%$ for $M_{\mathrm{VLQ}}=1 \mathrm{TeV}$, but still $\left|S\left(B_{s} \rightarrow \mu \bar{\mu}\right)\right|$ can reach up to $80 \%$. For $M_{\mathrm{VLQ}}=10 \mathrm{TeV}$, the former is restricted to positive values in both $\mathrm{LH}$ and $\mathrm{RH}$ models, the latter slightly stronger constrained in RH models, but not in LH ones. Of course, the experimental measurements are very challenging for $S\left(B_{s} \rightarrow \mu \bar{\mu}\right)$. We note that to very good accuracy $A_{\Delta \Gamma}^{2}+S^{2}=1$, since the direct CP-asymmetry $C\left(B_{s} \rightarrow \mu \bar{\mu}\right)$ is negligible.

- CP-violating quantities are almost $100 \%$ correlated in $b \rightarrow s$ transitions as long as only one representation is considered. The reason is that the SM predictions are tiny and all NP contributions therefore directly proportional to the imaginary part of $\Lambda_{b s}$, which hence cancels in the ratio of two CP-violating quantities. For small NP contributions, the asymmetries are simply proportional to each other, for larger effects the relation depends on the normalisation of the asymmetry. These statements hold not only in VLQ models, but in all models that provide only a single new phase in $b \rightarrow s$ transitions, only the proportionality constant changes in other models. 
- The imaginary parts of $b \rightarrow s \mu \bar{\mu}$ Wilson coefficients $C_{9,9^{\prime}, 10,10^{\prime}}$ can give rise to naive T-odd CP-asymmetries $A_{7,8,9}$ in $B \rightarrow K^{*} \mu \bar{\mu}$ that are tiny in the SM. ${ }^{15}$ The rough dependences on the Wilson coefficients are [89]

$$
A_{7} \propto \operatorname{Im}\left[\left(C_{10}-C_{10}^{\prime}\right) C_{7}^{*}\right], \quad A_{8,9} \propto \operatorname{Im}\left[C_{9} C_{9}^{*}+C_{10} C_{10}^{*}+\ldots\right],
$$

where the dots indicate other numerically suppressed interference terms of $C_{9,9^{\prime}}$ with $C_{7}$ that are included in the numerical evaluation. The $A_{7}$ remains tiny at high dilepton invariant mass $q^{2}$ [90]. These CP-asymmetries have been measured in various $q^{2}$-bins by LHCb [86] and we choose $q^{2} \in[1,6]$ and $[15,19] \mathrm{GeV}^{2}$, which have smallest experimental and theoretical uncertainties. As can be seen in table 9, the largest VLQ-effects in $A_{8,9}$ arise in $\mathrm{RH} \mathrm{G}_{\mathrm{SM}^{-} \text {-scenarios }} Q_{d}$ and $Q_{V}$, almost independent from the VLQ mass and with a strong anti-correlation shown in figure 8. The potential size of VLQ effects exceeds slightly the current experimental uncertainties, specifically for the CP asymmetry $A_{7}$ in LH scenarios, such that improved measurements will provide additional bounds on VLQ couplings in the future, especially on their imaginary parts. $A_{7}$ is correlated with $A_{8}$ and anti-correlated with $A_{9}$ in $\mathrm{RH}$ scenarios, whereas in LH scenarios $A_{8,9}$ remain SM-like.

- The decays $B \rightarrow K^{(*)} \nu \bar{\nu}$ are also sensitive probes of LH and RH NP effects due to $Z$-exchange and in order to exhibit these effects we consider the ratios [91]

$$
\epsilon=\frac{\sqrt{\left|C_{L}\right|^{2}+\left|C_{R}\right|^{2}}}{\left|C_{L}^{\mathrm{SM}}\right|} \quad \text { and } \quad \eta=\frac{-\operatorname{Re}\left(C_{L} C_{R}^{*}\right)}{\left|C_{L}\right|^{2}+\left|C_{R}\right|^{2}}
$$

which are unity and zero in the SM, respectively, and which determine the observables

$$
\mathcal{R}_{B \rightarrow K^{(*)} \nu \bar{\nu}}=\frac{\operatorname{Br}\left(B \rightarrow K^{(*)} \nu \bar{\nu}\right)}{B r\left(B \rightarrow K^{(*)} \nu \bar{\nu}\right)_{\mathrm{SM}}}, \quad \mathcal{R}_{F_{L}}=\frac{F_{L}\left(B \rightarrow K^{*} \nu \bar{\nu}\right)}{F_{L}\left(B \rightarrow K^{*} \nu \bar{\nu}\right)_{\mathrm{SM}}}
$$

via $[92]$

$$
\mathcal{R}_{B \rightarrow K \nu \bar{\nu}}=(1-2 \eta) \epsilon^{2}, \quad \mathcal{R}_{B \rightarrow K^{*} \nu \bar{\nu}}=\left(1+\kappa_{\eta} \eta\right) \epsilon^{2}, \quad \mathcal{R}_{F_{L}}=\frac{1+2 \eta}{1+\kappa_{\eta} \eta},
$$

where $\kappa_{\eta}$ is form-factor dependent and given in ref. [92]. The Belle II experiment is expected to measure these branching ratios with $30 \%$ uncertainty [93] if they are of the size as predicted in the SM. In RH scenarios large VLQ effects are excluded due to the strong complementarity of the $|\Delta F|=1$ constraints from $\operatorname{Br}\left(B_{s} \rightarrow \mu \bar{\mu}\right)$ and $\operatorname{Br}\left(B^{+} \rightarrow K^{+} \mu \bar{\mu}\right)$ as mentioned above. $\epsilon$ has to be larger than one in these cases. The VLQ effects for $M_{\mathrm{VLQ}}=1 \mathrm{TeV}$ can lead to a rather large suppression in LH scenarios for $\epsilon$ while $\eta=0$, leading to maximally correlated $\mathcal{R}_{B \rightarrow K^{(*)} \nu \bar{\nu}}$. The suppression is smaller for $M_{\mathrm{VLQ}}=10 \mathrm{TeV}$, whereas $\mathcal{R}_{F_{L}}=1$. The correlation plot is shown in figure 9 . It will be challenging to distinguish the small deviations from SM predictions in RH scenarios; however, large (suppression) effects are possible and LH and RH scenarios are well distinguishable. A measurement of $\epsilon$ significantly larger than one would challenge all $\mathrm{G}_{\mathrm{SM}}$ scenarios with a single VLQ representation.

\footnotetext{
${ }^{15}$ Note that we use different convention of angles w.r.t. LHCb: $A_{7,9}=-A_{7,9}^{\mathrm{LHCb}}$ and $A_{8}=A_{8}^{\mathrm{LHCb}}$.
} 


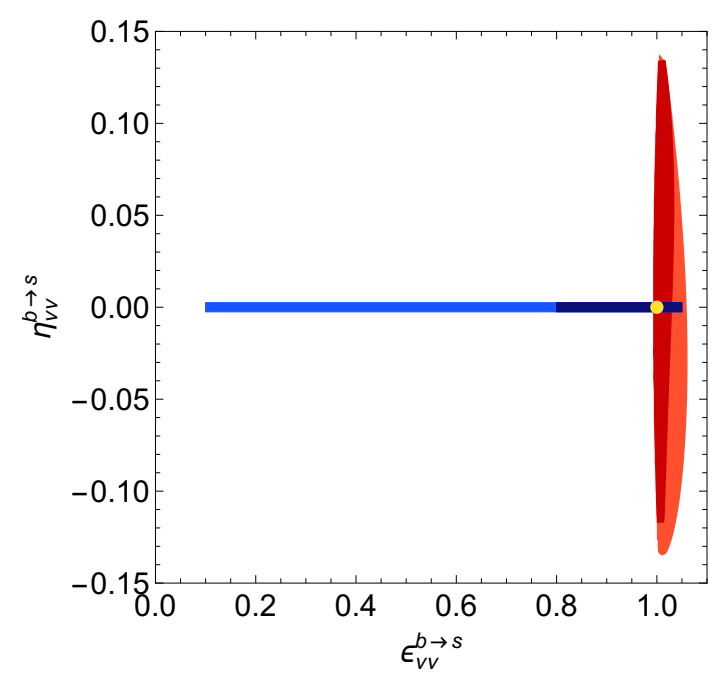

Figure 9. The correlations of the quantities $\eta$ and $\epsilon$, which determine the observables in the $b \rightarrow s \nu \bar{\nu}$ sector in $\mathrm{G}_{\mathrm{SM}}$-scenarios for $M_{\mathrm{VLQ}}=10 \mathrm{TeV}$ [darker] and $1 \mathrm{TeV}$ [lighter] within the $95 \%$ CL regions. The colours are for VLQ-representations $D$ [Blue] (similar to $\left.T_{u, d}\right), Q_{V}=Q_{d}$ [Red].

Similar correlation plots exist for $b \rightarrow d$ processes; however, given the CKM suppression of these modes compared to $b \rightarrow s$, precision measurements in $b \rightarrow d \ell \bar{\ell}$ and significant measurements of $b \rightarrow d \nu \bar{\nu}$ processes are not expected in the next couple of years. Nevertheless, we illustrate in figure 10 the impact of more precise measurements in this sector exemplarily for $\operatorname{Br}\left(B_{d} \rightarrow \mu \bar{\mu}\right)$. All $|\Delta F|=1$ processes depend only on the combination $\Delta_{i j}$, see (3.9), of NP parameters; the allowed range predicted from one $|\Delta F|=1$ process for another is therefore mass-independent, in contrast to the prediction from $|\Delta F|=2$ processes. The present measurement from the CMS and LHCb collaborations is about $2 \sigma$ larger than the SM prediction. As seen in figure 10 a confirmation of the present central value with higher precision would exclude $\mathrm{LH} \mathrm{G}_{\mathrm{SM}}$ scenarios and yield at least an upper limit on $M_{\mathrm{VLQ}}$ for the $\mathrm{RH}$ ones, in accordance with the discussion in section 5.3.

\section{$6.2 \quad \mathrm{G}_{\mathrm{SM}}^{\prime}(\Phi)$ model}

In $\mathrm{G}_{\mathrm{SM}}^{\prime}(\Phi)$ models $|\Delta F|=1$ transitions are suppressed by $\tan \beta$ compared to $\mathrm{G}_{\mathrm{SM}}$ models, such that $|\Delta F|=2$ transitions dominate via the box contributions the constraints on VLQ couplings. In our numerical analysis of $\mathrm{G}_{\mathrm{SM}}^{\prime}(\Phi)$ models we fix the parameters

$$
g^{\prime}=1.5, \quad X=1, \quad M_{\mathrm{VLQ}}=10 \mathrm{TeV},
$$

and choose two benchmark points BP1 and BP2:

$$
\begin{array}{lll}
\mathrm{BP} 1: & \tan \beta=2, & v_{S}=1.8 \mathrm{TeV}, \\
\mathrm{BP} 2: & \tan \beta=3, & v_{S}=1.3 \mathrm{TeV},
\end{array}
$$

in the lower range of possible values of $\tan \beta$ - see also figure 12 - from constraints described in appendix A.2 to maximally enhance VLQ contributions in $|\Delta F|=1$ transitions. 

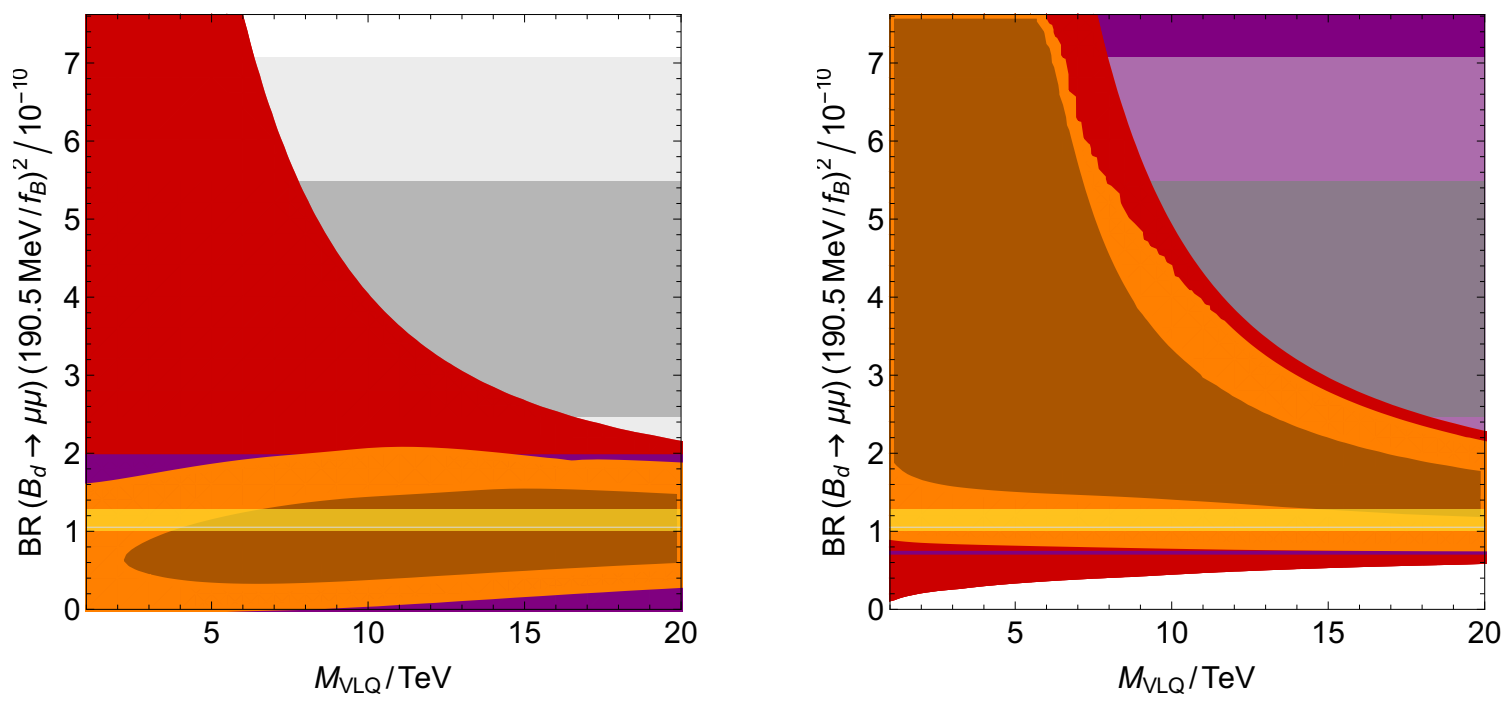

Figure 10. Predictions for $\operatorname{Br}\left(B_{d} \rightarrow \mu \bar{\mu}\right)$ for the LH G $\mathrm{G}_{\mathrm{SM}}$ scenario $D$ [left] and RH G $\mathrm{G}_{\mathrm{SM}}$ scenario $Q_{V}$ [right], in dependence on the VLQ mass. In dark red the constraint from $|\Delta F|=2$ processes is shown, i.e. $\Delta M_{d}$ and $\sin 2 \beta$, in purple the constraint from $B^{+} \rightarrow \pi^{+} \mu \bar{\mu}$, and in orange their combination. The yellow band corresponds to the SM prediction, the grey one to the measurement by the CMS and LHCb collaborations [77]. All constraints correspond to 95\% CL, only inner darker bands to $68 \%$ CL.

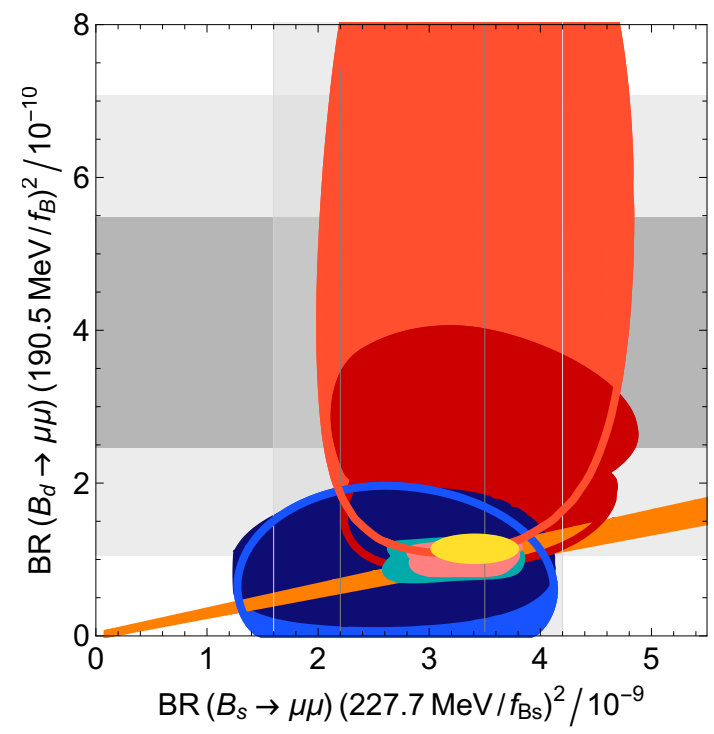

Figure 11. $\operatorname{Br}\left(B_{d} \rightarrow \mu \bar{\mu}\right)$ vs. $\operatorname{Br}\left(B_{s} \rightarrow \mu \bar{\mu}\right)$ in $\mathrm{G}_{\mathrm{SM}}$ at $10 \mathrm{TeV}$ [darker colours] and $1 \mathrm{TeV}$ (lighter colours), as well as $\mathrm{G}_{\mathrm{SM}}^{\prime}(\Phi)$ models at $10 \mathrm{TeV}$. The colours correspond to the representations $Q_{V}=Q_{d}$ [red and pink for $\mathrm{G}_{\mathrm{SM}}$ and $\left.\mathrm{G}_{\mathrm{SM}}^{\prime}(\Phi)\right]$ and $D$ [similar to $T_{u, d}$, blue and cyan]. The orange band corresponds to a scenario of constrained minimal flavour violation (CMFV) [92]. 


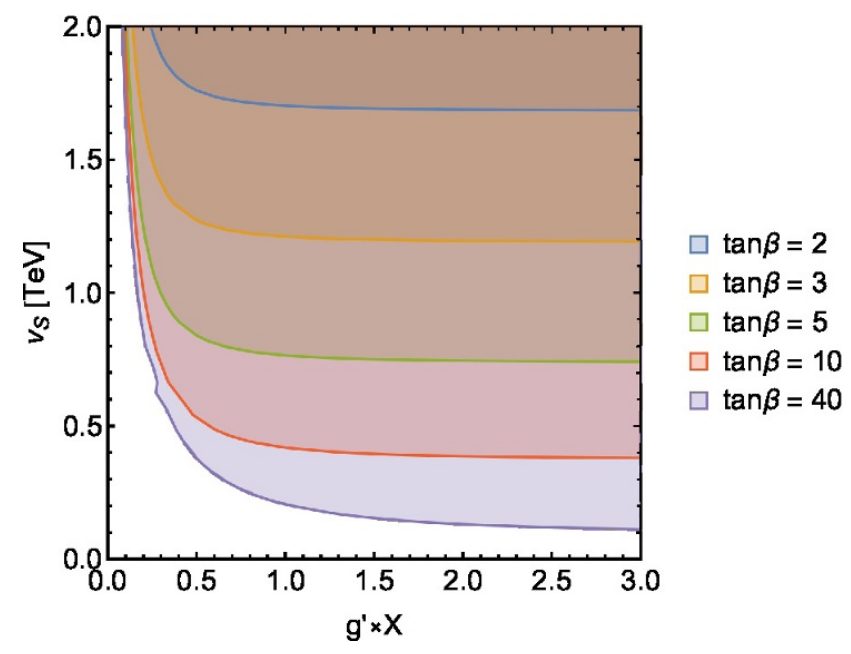

Figure 12. The allowed $95 \% \mathrm{CL}$ regions in the $v_{S}$ versus $g^{\prime} X$ plane for fixed $\tan \beta=2,3,5,10,40$ [Blue, Yellow, Green, Red, Purple] from the constraints $M_{Z}$ and partial widths $\Gamma[Z \rightarrow \ell \bar{\ell}](\ell=$ $e, \mu, \tau)$, imposing $M_{Z}<M_{Z^{\prime}}$.

The corresponding $Z$ and $Z^{\prime}$ masses and mixing angles are

$$
\begin{array}{llll}
\text { BP1: } & M_{Z}=91.51 \mathrm{GeV}, & M_{Z^{\prime}}=1.36 \mathrm{TeV}, & \xi_{Z Z^{\prime}}=0.0037 ; \\
\text { BP2: } & M_{Z}=91.58 \mathrm{GeV}, & M_{Z^{\prime}}=0.98 \mathrm{TeV}, & \xi_{Z Z^{\prime}}=0.0035 .
\end{array}
$$

The allowed regions of $\Lambda_{i j}$ in $\mathrm{G}_{\mathrm{SM}}^{\prime}(\Phi)$ models correspond to the regions allowed by $|\Delta F|=2$ constraints in $\mathrm{G}_{\mathrm{SM}}$ models given in figure 5 . We find that $|\Delta F|=1$ processes in table 8 provide only tiny additional constraints in $b \rightarrow d, s$ and small ones in $s \rightarrow d$, allowing thus in $\mathrm{G}_{\mathrm{SM}}^{\prime}(\Phi)$ models much larger values for $\Lambda_{i j}$ compared to $\mathrm{G}_{\mathrm{SM}}$ models.

The ranges still allowed for different observables with $|\Delta F|=1,2$ transitions are listed in table 10, obtained by varying $\Lambda_{i j}$ within the $95 \%$ CL regions, neglecting theory uncertainties. For this purpose $\left(\varepsilon^{\prime} / \varepsilon\right)_{\mathrm{NP}}$ has been restricted as given in eq. (6.1) and we used here $\operatorname{Br}\left(K_{L} \rightarrow \mu \bar{\mu}\right)_{\mathrm{SD}}<2.5 \times 10^{-9}$. Notable features for the benchmark points are:

- $\varepsilon^{\prime} / \varepsilon$ can also be enhanced in $\mathrm{G}_{\mathrm{SM}}^{\prime}(\Phi)$ models and thereby decrease the tension with the measurement. Especially in RH scenarios the constraint (6.1) is saturated, such that even larger effects are possible. The enhancement of $\varepsilon^{\prime} / \varepsilon$ falls off fast for larger values of $\tan \beta$ and $v_{S}$ than in the benchmark points.

- Whereas VLQ effects in $\operatorname{Br}\left(K_{L} \rightarrow \pi^{0} \nu \bar{\nu}\right)$ are small, $\operatorname{Br}\left(K^{+} \rightarrow \pi^{+} \nu \bar{\nu}\right)$ can still be enhanced over the SM prediction by a factor of two for LH and five for RH scenarios, while even larger effects are excluded by the upper bound on $\operatorname{Br}\left(K_{L} \rightarrow \mu \bar{\mu}\right)_{\mathrm{SD}}$. Most notably, $\left(\Delta M_{K}\right)_{\mathrm{SD}}$ can also be enhanced by a factor of more than two, in contradistinction to $\mathrm{G}_{\mathrm{SM}}$ models, where VLQ effects are tiny. The reason for this enhancement is the absence of strong constraints from $|\Delta F|=1$ on the real part of $\Lambda_{s d}$. Thus large $\left(\Delta M_{K}\right)_{\mathrm{SD}}$ is independent of $\varepsilon^{\prime} / \varepsilon$, since the latter is sensitive to the imaginary part of $\Lambda_{s d}$. This effect is enhanced with decreasing VLQ effects in $|\Delta S|=1$ transitions as can be seen by comparing the results for BP1 and BP2. 
- The VLQ effects are small for $\operatorname{Br}\left(B_{s, d} \rightarrow \mu \bar{\mu}\right)$ and $A_{\Delta \Gamma}\left(B_{s} \rightarrow \mu \bar{\mu}\right)$, as can be seen from figure 11 and table 10, respectively, but can be still sizeable for $S\left(B_{s} \rightarrow \mu \bar{\mu}\right)$. The CP asymmetries $A_{7,8,9}\left(B \rightarrow K^{*} \mu \bar{\mu}\right)$ can still be significantly enhanced over the SM to the percent level, but are a factor 2-3 smaller for BP1 than in $\mathrm{G}_{\mathrm{SM}}$ models, see table 9 .

- VLQ effects in $B \rightarrow K^{(*)} \nu \bar{\nu}$ in $\mathrm{G}_{\mathrm{SM}}^{\prime}(\Phi)$ models are smaller than in $\mathrm{G}_{\mathrm{SM}}$ models, at the level of only $(10-20) \%$ deviation from the SM predictions.

We provide a summary of enhancements and/or suppressions w.r.t. the SM predictions of the observables discussed above due to VLQ effects in table 11.

\section{Summary and conclusions}

In this paper we have analysed flavour-violation patterns in the $K$ and $B_{s, d}$ sectors in eleven models with vector-like quarks (VLQs). Five of them, called $\mathrm{G}_{\mathrm{SM}}$-models, contain only VLQs as new particles. Two of them, called $\mathrm{G}_{\mathrm{SM}}^{\prime}(S)$-models, have in addition a heavy $Z^{\prime}$ and a scalar $S$. The final four of them, called $\mathrm{G}_{\mathrm{SM}}^{\prime}(\Phi)$-models, contain a heavy $Z^{\prime}$, a scalar $S$ and a scalar doublet $\Phi$. Our summary of patterns of flavour violation in these models in section 5, accompanied by two DNA tables 6 and 7 and in particular our extensive numerical analysis in section 6 , see specifically tables 9 and 10, has shown that NP effects in several of these models can be still very large and that simultaneous consideration of several flavour observables should allow to distinguish between these models. This is also seen in table 11, which shows that models with LH currents can be distinguished from models with RH currents through several observables.

On the theoretical side our paper presents the first analysis of VLQ models in the context of SMEFT, which allowed to include RG effects from the NP scale $M_{\mathrm{VLQ}}$ down to the electroweak scale, thereby identifying very important Yukawa enhancement of NP contributions to $|\Delta F|=2$ observables in the Kaon sector through the generation of left-right operators with smaller, but significant effects in $B_{s, d}$ observables. These RG effects, relevant only in $\mathrm{G}_{\mathrm{SM}}$-models, have been already identified in general $Z$ models in [18], but in the present paper they could be studied explicitly in concrete models. The relevant technology is described in detail in [18] and in section 3, section 4 and appendix B of the present paper.

As our results have been systematically summarized in the previous section, we list here only the main highlights. Most interesting NP effects are found in $\mathrm{G}_{\mathrm{SM}}$-models, even if they do not provide the explanation of the present $\mathrm{LHCb}$ anomalies. In particular

- Tree-level $Z$ contributions to $\varepsilon^{\prime} / \varepsilon$ can be large, so that the apparent upward shift in $\varepsilon^{\prime} / \varepsilon$ can easily be obtained, bringing the theory to agree with data.

- Simultaneously the branching ratio for $K^{+} \rightarrow \pi^{+} \nu \bar{\nu}$ can be enhanced over its SM prediction, but the size of the enhancement depends on whether RH currents or LH currents are considered. In models with flavour-violating $\mathrm{RH}$ currents, the maximal enhancement is limited to $\sim 50 \%$ of its SM value because of the strong constraint from $\epsilon_{K}$, caused by RG-enhanced contributions. In the LH current case an enhancement of $K^{+} \rightarrow \pi^{+} \nu \bar{\nu}$ is only possible if the present conservative bound on $K_{L} \rightarrow \mu \bar{\mu}$ is used. 


\begin{tabular}{|c|c|c|c|c|c|}
\hline SM & measurement & $D$ & $Q_{d}$ & $T_{u}$ & $T_{d}$ \\
\hline \multicolumn{6}{|c|}{$10^{4} \times\left(\varepsilon^{\prime} / \varepsilon\right)_{\mathrm{NP}}$} \\
\hline$(4.17),(4.18),(4.20)$ & $(4.19)$ & $\begin{array}{l}{[0.0,20.0]} \\
{[0.0,20.0]}\end{array}$ & $\begin{array}{l}{[0.0,20.0]} \\
{[0.0,19.9]}\end{array}$ & $\begin{array}{l}{[0.0,20.0]} \\
{[0.0,20.0]}\end{array}$ & $\begin{array}{l}{[0.0,19.9]} \\
{[0.0,20.0]}\end{array}$ \\
\hline \multicolumn{6}{|c|}{$10^{11} \times \operatorname{Br}\left(K_{L} \rightarrow \pi^{0} \nu \bar{\nu}\right), \quad 10^{11} \times B r\left(K^{+} \rightarrow \pi+\nu \bar{\nu}\right)$} \\
\hline $3.2[2.5,4.3]$ & $\leq 2600$ & $\begin{array}{l}{[0.1,3.2]} \\
{[0.1,3.2]}\end{array}$ & $\begin{array}{l}{[1.8,3.2]} \\
{[1.8,3.2]}\end{array}$ & $\begin{array}{l}{[0.1,3.2]} \\
{[0.1,3.2]}\end{array}$ & $\begin{array}{l}{[0.1,3.2]} \\
{[0.9,3.2]}\end{array}$ \\
\hline $8.5[7.3,9.5]$ & $17.3_{-10.5}^{+11.5}$ & $\begin{array}{l}{[1.5,15.4]} \\
{[1.6,15.4]}\end{array}$ & $\begin{array}{l}{[3.9,45.4]} \\
{[3.9,45.4]}\end{array}$ & $\begin{array}{l}{[1.5,15.4]} \\
{[1.6,15.4]}\end{array}$ & $\begin{array}{l}{[1.5,15.4]} \\
{[1.6,15.4]}\end{array}$ \\
\hline \multicolumn{6}{|c|}{$10^{9} \times B r\left(K_{L} \rightarrow \mu \bar{\mu}\right)_{\mathrm{SD}}$} \\
\hline & $\leq 2.5$ & $\begin{array}{l}{[0.0,2.5]} \\
{[0.0,2.5]} \\
\end{array}$ & $\begin{array}{l}{[0.0,2.5]} \\
{[0.0,2.5]}\end{array}$ & $\begin{array}{l}{[0.0,2.5]} \\
{[0.0,2.5]}\end{array}$ & $\begin{array}{l}{[0.0,2.5]} \\
{[0.0,2.5]}\end{array}$ \\
\hline \multicolumn{6}{|c|}{$10^{4} \times\left(\Delta M_{K}\right)_{\mathrm{SD}}\left[\mathrm{ps}^{-1}\right]$} \\
\hline & $52.93 \pm 0.09 \quad[72]$ & $\begin{array}{l}{[46.0,58.8]} \\
{[44.7,95.7]}\end{array}$ & $\begin{array}{l}{[46.4,71.1]} \\
{[46.1,144.9]}\end{array}$ & $\begin{array}{l}{[45.9,61.9]} \\
{[44.2,105.9]}\end{array}$ & $\begin{array}{l}{[44.2,105.9]} \\
{[44.5,161.0]}\end{array}$ \\
\hline \multicolumn{6}{|c|}{$10^{10} \times \operatorname{Br}\left(B_{d} \rightarrow \mu \bar{\mu}\right)$} \\
\hline $1.14[0.94,1.32]$ & $\leq 6.3$ & $\begin{array}{l}{[0.74,1.32]} \\
{[0.90,1.21]} \\
\end{array}$ & $\begin{array}{l}{[0.90,1.34]} \\
{[0.97,1.19]}\end{array}$ & $\begin{array}{l}{[0.77,1.30]} \\
{[0.92,1.19]} \\
\end{array}$ & $\begin{array}{l}{[0.92,1.20]} \\
{[1.00,1.13]} \\
\end{array}$ \\
\hline \multicolumn{6}{|c|}{$A_{\Delta \Gamma}\left(B_{s} \rightarrow \mu \bar{\mu}\right), \quad S\left(B_{s} \rightarrow \mu \bar{\mu}\right)$} \\
\hline 1 & & $\begin{array}{l}{[0.88,1.00]} \\
{[0.97,1.00]}\end{array}$ & $\begin{array}{l}{[0.95,1.00]} \\
{[0.98,1.00]}\end{array}$ & $\begin{array}{l}{[0.91,1.00]} \\
{[0.98,1.00]}\end{array}$ & $\begin{array}{l}{[0.97,1.00]} \\
{[0.99,1.00]}\end{array}$ \\
\hline 0 & - & $\begin{array}{l}{[-0.47,0.46]} \\
{[-0.25,0.25]}\end{array}$ & $\begin{array}{l}{[-0.34,0.34]} \\
{[-0.18,0.18]}\end{array}$ & $\begin{array}{l}{[-0.43,0.42]} \\
{[-0.22,0.22]}\end{array}$ & $\begin{array}{l}{[-0.22,0.22]} \\
{[-0.11,0.11]}\end{array}$ \\
\hline \multicolumn{6}{|c|}{$10^{2} \times A_{7,8,9}\left(B \rightarrow K^{*} \mu \bar{\mu}\right)_{[1,6]}$} \\
\hline$<0.1$ & $4.5 \pm 5.0 \quad[86]$ & $\begin{array}{l}{[-5.0,5.1]} \\
{[-2.6,2.7]}\end{array}$ & $\begin{array}{l}{[-3.7,3.7]} \\
{[-1.9,1.9]}\end{array}$ & $\begin{array}{l}{[-4.5,4.6]} \\
{[-2.4,2.4]}\end{array}$ & $\begin{array}{l}{[-2.4,2.4]} \\
{[-1.2,1.2]}\end{array}$ \\
\hline$<0.1$ & $-4.7 \pm 5.8 \quad[86]$ & $\begin{array}{l}{[-0.6,0.5]} \\
{[-0.5,0.4]}\end{array}$ & $\begin{array}{l}{[-3.0,2.9]} \\
{[-1.6,1.6]}\end{array}$ & $\begin{array}{l}{[-0.5,0.5]} \\
{[-0.4,0.4]}\end{array}$ & $\begin{array}{l}{[-0.3,0.2]} \\
{[-0.2,0.2]}\end{array}$ \\
\hline$<0.1$ & $3.3 \pm 4.2 \quad[86]$ & $\mathrm{SM}$ & $\begin{array}{l}{[-1.7,1.7]} \\
{[-0.9,0.9]}\end{array}$ & SM & SM \\
\hline & $10^{2} \times A_{8,9}$ & $\rightarrow K^{*} \mu \bar{\mu}$ & $5,19]$ & & \\
\hline$<0.1$ & $2.5 \pm 4.8 \quad[86]$ & $\mathrm{SM}$ & $\begin{array}{l}{[-2.4,2.4]} \\
{[-1.4,1.4]}\end{array}$ & $\mathrm{SM}$ & $\mathrm{SM}$ \\
\hline$<0.1$ & $-6.1 \pm 4.3$ & $\mathrm{SM}$ & $\begin{array}{l}{[-4.4,4.4]} \\
{[-2.4,2.5]} \\
\end{array}$ & SM & $\mathrm{SM}$ \\
\hline 1 & $\begin{array}{rr} & \mathcal{R}_{B \rightarrow K \nu \bar{\nu}} \\
\leq 4.3 & {[87]}\end{array}$ & $\begin{array}{c}\mathcal{R}_{B \rightarrow K^{*} \nu \bar{\nu}}, \\
{[0.78,1.13]} \\
{[0.88,1.09]}\end{array}$ & $\begin{array}{l}\mathcal{R}_{F_{L}} \\
{[0.87,1.15]} \\
{[0.93,1.08]}\end{array}$ & $\begin{array}{l}{[0.80,1.13]} \\
{[0.89,1.08]}\end{array}$ & $\begin{array}{l}{[0.90,1.08]} \\
{[0.95,1.05]}\end{array}$ \\
\hline 1 & $\leq 4.4$ & $\begin{array}{l}{[0.78,1.13]} \\
{[0.88,1.09]}\end{array}$ & $\begin{array}{l}{[0.91,1.10]} \\
{[0.95,1.05]}\end{array}$ & $\begin{array}{l}{[0.80,1.13]} \\
{[0.90,1.08]}\end{array}$ & $\begin{array}{l}{[0.90,1.08]} \\
{[0.95,1.05]}\end{array}$ \\
\hline 1 & - & $\mathrm{SM}$ & $\begin{array}{l}{[0.95,1.04]} \\
{[0.97,1.02]} \\
\end{array}$ & SM & $\mathrm{SM}$ \\
\hline
\end{tabular}

Table 10. Ranges still allowed for observables when varying $\Lambda_{i j}$ of $\mathrm{G}_{\mathrm{SM}}^{\prime}(\Phi)$ models in the $95 \% \mathrm{CL}$ ranges for individual $s \rightarrow d, b \rightarrow d$ and $b \rightarrow s$ sectors for benchmark points BP1/BP2 [upper/lower]. Moreover $\left(\varepsilon^{\prime} / \varepsilon\right)_{\mathrm{NP}}$ is restricted as given in eq. (6.1). Entries denoted as "SM" have tiny or no deviations from the SM. Experimental upper bounds are given at $90 \%$ CL. 


\begin{tabular}{|c|c|c|c|c|c|c|c|c|}
\hline & \multicolumn{4}{|c|}{$\mathrm{G}_{\mathrm{SM}}$} & \multicolumn{4}{|c|}{$\mathrm{G}_{\mathrm{SM}}^{\prime}(\Phi)$} \\
\hline & $D$ & $Q_{V, d}$ & $T_{d}$ & $T_{u}$ & $D$ & $Q_{d}$ & $T_{d}$ & $T_{u}$ \\
\hline$\Delta M_{K}$ & & & & & $\Uparrow$ & $\Uparrow$ & $\Uparrow$ & $\Uparrow$ \\
\hline$\varepsilon^{\prime} / \varepsilon$ & $\Uparrow$ & $\Uparrow$ & $\Uparrow$ & $\Uparrow$ & $\Uparrow$ & $\Uparrow$ & $\Uparrow$ & $\Uparrow$ \\
\hline$K^{+} \rightarrow \pi^{+} \nu \bar{\nu}$ & $\Downarrow$ & $\Uparrow$ & $\Downarrow$ & $\Downarrow$ & $\mathbb{1}$ & $\Uparrow$ & $\mathbb{1}$ & $\mathbb{1}$ \\
\hline$K_{L} \rightarrow \pi^{0} \nu \bar{\nu}$ & $\Downarrow$ & $\Downarrow$ & $\Downarrow$ & $\Downarrow$ & $\Downarrow$ & $\Downarrow$ & $\Downarrow$ & $\Downarrow$ \\
\hline$B r\left(B_{d} \rightarrow \mu \bar{\mu}\right)$ & $\mathbb{1}$ & $\Uparrow$ & $\mathbb{1}$ & $\mathbb{1}$ & & & & \\
\hline$B r\left(B_{s} \rightarrow \mu \bar{\mu}\right)$ & $\Uparrow$ & $\Uparrow$ & $\Uparrow$ & $\Uparrow$ & & & & \\
\hline$A_{\Delta \Gamma}\left(B_{s} \rightarrow \mu \bar{\mu}\right)$ & $\Downarrow$ & $\Downarrow$ & $\Downarrow$ & $\Downarrow$ & & & & \\
\hline$S\left(B_{s} \rightarrow \mu \bar{\mu}\right)$ & $\mathbb{1}$ & $\Uparrow$ & $\mathbb{I}$ & $\mathbb{\Downarrow}$ & $\mathbb{1}$ & $\mathbb{\Downarrow}$ & $\Uparrow$ & $\mathbb{1}$ \\
\hline$A_{7}\left(B \rightarrow K^{*} \mu \bar{\mu}\right)_{[1,6]}$ & $\Uparrow$ & $\Uparrow$ & $\mathbb{I}$ & $\Uparrow$ & $\mathbb{1}$ & $\Uparrow$ & $\mathbb{1}$ & $\mathbb{1}$ \\
\hline$A_{8,9}\left(B \rightarrow K^{*} \mu \bar{\mu}\right)_{[1,6]}$ & & $\Uparrow$ & & & & $\mathbb{1}$ & & \\
\hline$A_{8,9}\left(B \rightarrow K^{*} \mu \bar{\mu}\right)_{[15,19]}$ & & $\Uparrow$ & & & & $\mathbb{1}$ & & \\
\hline $\mathcal{R}_{B \rightarrow K \nu \bar{\nu}}$ & $\Downarrow$ & $\Uparrow$ & $\Downarrow$ & $\Downarrow$ & & & & \\
\hline $\mathcal{R}_{B \rightarrow K^{*} \nu \bar{\nu}}$ & $\Downarrow$ & $\Uparrow$ & $\Downarrow$ & $\Downarrow$ & & & & \\
\hline $\mathcal{R}_{F_{L}}$ & & $\Downarrow$ & & & & & & \\
\hline
\end{tabular}

Table 11. Summary of allowed VLQ effects in $\mathrm{G}_{\mathrm{SM}^{-}}$and $\mathrm{G}_{\mathrm{SM}}^{\prime}(\Phi)$-models in flavour observables after the fit using experimental measurements of table 8. Possible enhancement, suppression or both w.r.t. SM predictions are indicated by according $\Uparrow$, $\Downarrow$ or $\mathbb{\Downarrow}$. Empty space means that the given model does not predict sizeable effects in that observable. Note that $\left(\varepsilon^{\prime} / \varepsilon\right)_{\mathrm{NP}}$ has been restricted (6.1), affecting other $s \rightarrow d$ observables.

With the stricter bound only suppression of $K^{+} \rightarrow \pi^{+} \nu \bar{\nu}$ is possible. On the other hand the positive shift in $\varepsilon^{\prime} / \varepsilon$ implies uniquely the suppression of the $K_{L} \rightarrow \pi^{0} \nu \bar{\nu}$ branching ratio.

- Potential tensions between $\Delta M_{s, d}$ and $\varepsilon_{K}$ can be easily removed in these models, since no MFV relation is imposed on the couplings.

- Significant suppressions of the $\operatorname{Br}\left(B_{s} \rightarrow \mu \bar{\mu}\right)$ and of $A_{\Delta \Gamma}\left(B_{s} \rightarrow \mu \bar{\mu}\right)$, in particular in models with LH currents, are possible. As far as $\operatorname{Br}\left(B_{d} \rightarrow \mu \bar{\mu}\right)$ is concerned, significant enhancements, in particular in the $\mathrm{RH}$ current scenarios, are still possible, as seen in figure 10 and figure 11. While such effects are also possible in 331 models, they cannot be as large as in VLQ models. 
- CP-violating effects for a given quark transition are strongly correlated in all of theses models, as long as only one representation is present, specifically for $b \rightarrow s$, where $\mathrm{CP}$ violation in the $\mathrm{SM}$ is tiny.

Having the LHCb anomalies in mind we have considered also VLQ models with a heavy $Z^{\prime}$ related to $\mathrm{U}(1)_{\mathrm{L}_{\mu}-\mathrm{L}_{\tau}}$ symmetry. Our finding are as follows:

- The $\mathrm{G}_{\mathrm{SM}}^{\prime}(S)$-models, considered already in ref. [9], can explain the LHCb anomalies by providing sufficient suppression of the coefficient $C_{9}$, but NP effects in $B_{s, d} \rightarrow \mu \bar{\mu}$ and $K_{L} \rightarrow \mu \bar{\mu}$ are absent, those in $b \rightarrow s \nu \bar{\nu}$ transitions small and the ones in $K^{+} \rightarrow$ $\pi^{+} \nu \bar{\nu}$ and $K_{L} \rightarrow \pi^{0} \nu \bar{\nu}$ much smaller than in $\mathrm{G}_{\mathrm{SM}}$-models. Most importantly these models fail badly in explaining the $\varepsilon^{\prime} / \varepsilon$ anomaly.

- In the $\mathrm{G}_{\mathrm{SM}}^{\prime}(\Phi)$-models, the explanation of LHCb anomalies is more difficult than in $\mathrm{G}_{\mathrm{SM}}^{\prime}(S)$-models, but this time, due to the presence of $Z$ contributions, interesting effects in other observables can be found.

- In particular, in contrast to $\mathrm{G}_{\mathrm{SM}}$-models, the parametric suppression of $Z$ couplings by $\tan \beta$ allows for increased values of Yukawa couplings that are this time mainly bounded by $|\Delta F|=2$ transitions.

- We find that NP effects in $\varepsilon^{\prime} / \varepsilon$ and $K^{+} \rightarrow \pi^{+} \nu \bar{\nu}$ can be large, the latter in contrast to $\mathrm{G}_{\mathrm{SM}}$-models, and also the corresponding effects in $\Delta M_{K}$ can be significantly larger than in $\mathrm{G}_{\mathrm{SM}}$-models. This could appear in contradiction with the pattern in table 7 and is the result of weaker constraints in these models. In particular if in the future the $\Delta M_{K}$ constraint will be improved, such large enhancements of $\operatorname{Br}\left(K^{+} \rightarrow \pi^{+} \nu \bar{\nu}\right)$ are likely to be excluded. On the other hand NP effects in $K_{L} \rightarrow \pi^{0} \nu \bar{\nu}, K_{L} \rightarrow \mu \bar{\mu}$, $B \rightarrow K\left(K^{*}\right) \nu \bar{\nu}$ and $B_{d, s} \rightarrow \mu \bar{\mu}$ are very small and beyond the reach of even presently planned future facilities. While effects in the CP asymmetries $A_{7,8,9}\left(B \rightarrow K^{*} \mu \bar{\mu}\right)$ are smaller than in $\mathrm{G}_{\mathrm{SM}}$ models, they might be still within reach of $\mathrm{LHCb}$.

Thus if NP will be found in $B_{s, d} \rightarrow \mu \bar{\mu}$ and the $\varepsilon^{\prime} / \varepsilon$-anomaly will be confirmed by future lattice data, $\mathrm{G}_{\mathrm{SM}}$-models would offer the best explanation among VLQ models. If, on the other hand, the LHCb anomalies will be confirmed in the future and no visible NP will be found in rare $K$ decays, $\mathrm{G}_{\mathrm{SM}}^{\prime}(S)$-models and $\mathrm{G}_{\mathrm{SM}}^{\prime}(\Phi)$-models would be favoured over $\mathrm{G}_{\mathrm{SM}}$-models. A large enhancement of $\operatorname{Br}\left(K^{+} \rightarrow \pi^{+} \nu \bar{\nu}\right)$ would uniquely select $\mathrm{RH} \mathrm{G}_{\mathrm{SM}}^{\prime}(\Phi)$ models subject to the future status of $\Delta M_{K}$, although LH $\mathrm{G}_{\mathrm{SM}}$ and $\mathrm{G}_{\mathrm{SM}}^{\prime}(\Phi)$ models could provide a moderate enhancement, in case of the latter depending on the theoretical treatment of $K_{L} \rightarrow \mu \bar{\mu}$. On the other hand, a large enhancement of $\operatorname{Br}\left(B \rightarrow K^{(*)} \nu \bar{\nu}\right)$ would disfavour all considered models, at least with only one VLQ representation. Also the confirmation of all anomalies in combination with sizeable effects in e.g. $\operatorname{Br}\left(B_{d, s} \rightarrow \mu \bar{\mu}\right)$ would force us to extend the models analyzed by us by considering several VLQ representations simultaneously. We have also pointed out that in $\mathrm{G}_{\mathrm{SM}}^{\prime}(\Phi)$-models significant NP effects in $\Delta M_{K}$ can be found, larger than in $\mathrm{G}_{\mathrm{SM}}$ and $\mathrm{G}_{\mathrm{SM}}^{\prime}(S)$-models.

While the discovery of VLQs at the LHC would give a strong impetus to the models considered by us, non-observation of them at the LHC would not preclude their importance 
for flavour physics. In fact, as we have shown, large NP effects in flavour observables can be present for $M_{\mathrm{VLQ}}=10 \mathrm{TeV}$ and in the flavour-precision era one is sensitive to even higher scales. In this context we have pointed out that the combination of $|\Delta F|=2$ and $|\Delta F|=1$ observables in a given meson system generally allows to determine the masses of VLQs in a given representation independently of the size of Yukawa couplings.

\section{Acknowledgments}

C.B. thanks Martin Gorbahn for numerical checks on $\varepsilon^{\prime} / \varepsilon$. We thank Sebastien DescotesGenon for providing us an update of a tree-level CKM fit from CKMfitter [94]. This research was done and financed in the context of the ERC Advanced Grant project "FLAVOUR" (267104) and was partially supported by the DFG cluster of excellence "Origin and Structure of the Universe". The work of A.C. is supported by the Alexander von Humboldt Foundation. This work is supported in part by the DFG SFB/TR 110 "Symmetries and the Emergence of Structure in QCD".

\section{A Scalar sectors of $\mathrm{G}_{\mathrm{SM}^{\prime}}^{- \text {models }}$}

\section{A.1 $\quad \mathrm{G}_{\mathrm{SM}}^{\prime}(S)$ models}

The scalar sector in $\mathrm{G}_{\mathrm{SM}}^{\prime}(S)$-models with one complex scalar $S(1,0, X)$ and the SM doublet $H(2,+1 / 2,0)$ is given by

$$
\mathcal{L}=\left|\mathcal{D}_{\mu} H\right|^{2}+\left|\mathcal{D}_{\mu} S\right|^{2}-V
$$

with the potential

$$
V=m^{2} H^{\dagger} H+\frac{\lambda}{2}\left(H^{\dagger} H\right)^{2}+\frac{b_{2}}{2}|S|^{2}+\frac{d_{2}}{4}|S|^{4}+\frac{\delta}{2} H^{\dagger} H|S|^{2} .
$$

We parametrise the SM Higgs doublet and the complex scalar as

$$
H=\left(\begin{array}{c}
H^{+} \\
H^{0}
\end{array}\right)=\left(\begin{array}{c}
G^{+} \\
\left(v+h^{0}+i G^{0}\right) / \sqrt{2}
\end{array}\right), \quad S=\frac{\left(v_{S}+R_{0}+i I_{0}\right)}{\sqrt{2}} .
$$

The neutral mass-eigenstates are given by $(h, H)^{T} \simeq\left(h^{0}, R_{0}\right)^{T}$ with approximate masses

$$
m_{h}^{2} \approx v^{2}\left(\lambda-\frac{\delta^{2}}{2 d_{2}}\right), \quad m_{H}^{2} \approx v_{S}^{2} \frac{d_{2}}{2},
$$

up to terms $\mathcal{O}\left(v^{2} / v_{S}^{2}\right)$. The general expressions can be found in [95].

Kinetic mixing of $Z$ and $Z^{\prime}$ is caused by VLQ-exchange and depends on the VLQ

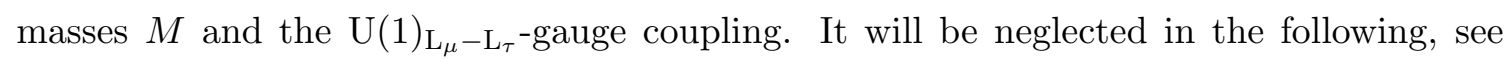
ref. [9]. Mass mixing does not occur in $\mathrm{G}_{\mathrm{SM}}^{\prime}(S)$ models. 


\section{A.2 $\mathrm{G}_{\mathrm{SM}}^{\prime}(\Phi)$ models}

The scalar sector in $\mathrm{G}_{\mathrm{SM}}^{\prime}(\Phi)$-models with one complex scalar $S(1,0, X / 2)$ and the two doublets $\Phi_{1} \equiv \Phi(2,+1 / 2, X)$ and $\Phi_{2} \equiv H(2,+1 / 2,0)$ is given by

$$
\mathcal{L}=\left|\mathcal{D}_{\mu} \Phi_{1}\right|^{2}+\left|\mathcal{D}_{\mu} \Phi_{2}\right|^{2}+\left|\mathcal{D}_{\mu} S\right|^{2}-V
$$

with the potential

$$
\begin{aligned}
V= & m_{a}^{2} \Phi_{a}^{\dagger} \Phi_{a}+\frac{\lambda_{a}}{2}\left(\Phi_{a}^{\dagger} \Phi_{a}\right)^{2}+\lambda_{3}\left(\Phi_{1}^{\dagger} \Phi_{1}\right)\left(\Phi_{2}^{\dagger} \Phi_{2}\right)+\lambda_{4}\left(\Phi_{1}^{\dagger} \Phi_{2}\right)\left(\Phi_{2}^{\dagger} \Phi_{1}\right) \\
& +\frac{b_{2}}{2}|S|^{2}+\frac{d_{2}}{4}|S|^{4}+\frac{\delta_{a}}{2} \Phi_{a}^{\dagger} \Phi_{a}|S|^{2}-\frac{\delta_{3}}{4}\left[\Phi_{1}^{\dagger} \Phi_{2} S^{2}+\Phi_{2}^{\dagger} \Phi_{1}\left(S^{*}\right)^{2}\right] .
\end{aligned}
$$

We neglect kinetic mixing and parametrise the mass mixing via

$$
\left(\begin{array}{l}
\hat{Z}_{\mu} \\
\hat{Z}_{\mu}^{\prime}
\end{array}\right)=\left(\begin{array}{cc}
\cos \xi_{Z Z^{\prime}} & -\sin \xi_{Z Z^{\prime}} \\
\sin \xi_{Z Z^{\prime}} & \cos \xi_{Z Z^{\prime}}
\end{array}\right)\left(\begin{array}{c}
Z_{\mu} \\
Z_{\mu}^{\prime}
\end{array}\right) .
$$

After partial diagonalization of the neutral gauge boson system, the $Z$ and $Z^{\prime}$ masses and their mass mixing are given by [96]

$$
\hat{M}_{Z}^{2}=g_{Z}^{2} \frac{v^{2}}{4}, \quad \hat{M}_{Z^{\prime}}^{2}=\left(g^{\prime} X\right)^{2} \frac{v_{S}^{2}}{4}\left(1+4 c_{\beta}^{2} \frac{v^{2}}{v_{S}^{2}}\right), \quad \Delta^{2}=-g_{Z} g^{\prime} X c_{\beta}^{2} \frac{v^{2}}{2},
$$

with $e=\sqrt{4 \pi \alpha}=g_{2} \hat{s}_{W}=g_{1} \hat{c}_{W}=g_{Z} \hat{s}_{W} \hat{c}_{W}$. The $Z-Z^{\prime}$ mixing angle

$$
\tan 2 \xi_{Z Z^{\prime}}=\frac{2 \Delta^{2}}{\hat{M}_{Z}^{2}-\hat{M}_{Z^{\prime}}^{2}}=c_{\beta}^{2} \frac{4 X g^{\prime}}{g_{Z}} \frac{\hat{M}_{Z}^{2}}{\left(\hat{M}_{Z^{\prime}}^{2}-\hat{M}_{Z}^{2}\right)}
$$

is small unless $X$ becomes large. The diagonalisation of the neutral gauge boson mass matrix gives mass eigenvalues

$$
M_{Z, Z^{\prime}}^{2}=\frac{1}{2}\left[\hat{M}_{Z^{\prime}}^{2}+\hat{M}_{Z}^{2} \mp \sqrt{\left(\hat{M}_{Z^{\prime}}^{2}-\hat{M}_{Z}^{2}\right)^{2}+4 \Delta^{4}}\right],
$$

which differ from the ones in eq. (A.8) by terms $\mathcal{O}\left(v^{2} / v_{S}^{2}\right)$. Note that we present only the solution for which $M_{Z}<M_{Z^{\prime}}$, i.e. throughout we will implicitly impose that the lighter mass eigenstate couples predominantly SM-like to quarks and leptons. As a consequence a lower bound on $g^{\prime}$ will be obtained. On the other hand, the decoupling limit $g^{\prime} \rightarrow 0$ is not excluded, but it will lead to $M_{Z^{\prime}}<M_{Z}$, i.e. that the heavier mass-eigenstate couples predominantly to SM-like fermions. The $\tan \beta$ dependence of $M_{Z^{\prime}}$ becomes irrelevant once $v_{S} \gtrsim$ $0.5 \mathrm{TeV}$. The mixing angle $\xi_{Z Z^{\prime}}$ can be suppressed with large $\tan \beta$ and $M_{Z^{\prime}}$, since we work in the part of the parameter space, where the other possibility of $g^{\prime} \rightarrow 0$ is not an option.

In $\mathrm{G}_{\mathrm{SM}}^{\prime}(\Phi)$-models we make use of the fact that photon- and $W^{ \pm}$-interactions to leptons are SM-like in order to determine the values of the fundamental gauge couplings $g_{1,2}$ and the VEV $v$ from $\alpha_{e}\left(M_{Z}\right), G_{F}$ and the $W$-boson pole mass $M_{W}$. As the remaining free parameters we choose $\tan \beta, g^{\prime}, X$ and $v_{S}$, whereas dependent parameters are $M_{Z, Z^{\prime}}$ and 
$\xi_{Z Z^{\prime}}$. Note that the latter depend only on the product $g^{\prime} X$, such that there are effectively only three parameters. We will restrict this parameter space to

$$
0.3 \leq \tan \beta \leq 40, \quad 0 \leq g^{\prime} X \leq 3, \quad 0 \mathrm{TeV} \leq v_{S} \leq 2 \mathrm{TeV} .
$$

The lower bound on $\tan \beta$ guarantees perturbativity of the top-quark Yukawa coupling [64], whereas $v_{S}$ is bounded from above by the requirements (3.1) and yields $M_{Z^{\prime}} \lesssim 1.5 \mathrm{TeV}$ within the above limits. Constraints on these parameters arise from the measured value of $M_{Z}$, which we impose with an error of $\delta M_{Z}=5 \mathrm{GeV}$ to account for the use of tree-level relations only. Further constraints come from the partial widths of $Z \rightarrow \ell \bar{\ell}(\ell=e, \mu, \tau)$, constraining the new physics contributions of the $Z$-lepton couplings (3.11) that depend on the $\xi_{Z Z^{\prime}}$ and $g^{\prime}$ due to gauge mixing. We find a small mixing angle $\xi_{Z Z^{\prime}} \lesssim 0.1$ in the above specified parameter space of $\tan \beta, g^{\prime} X$ and $v_{S}$ if we impose the bound on new physics contributions to the partial widths of $Z \rightarrow \ell \bar{\ell}$ from LEP [24], allowing for $5 \sigma$ deviations from the measured central values, together with the bound on $M_{Z}$. This justifies the expansion in the small mixing angle as done in table 4 .

\section{B VLQ decoupling and RG effects}

This appendix contains results of the Wilson coefficients of $\psi^{2} \varphi^{2} D$ and $\psi^{2} \varphi^{3}$ operators in $\mathrm{G}_{\mathrm{SM}}^{(\prime)}$-EFTs after the tree-level decoupling of VLQs at the scale $\mu_{M}$. We provide further the relations to flavour-changing $Z$ and $Z^{\prime}$ couplings (3.7) and (3.8) after spontaneous symmetry breaking at the scale $\mu_{\mathrm{EW}}$ (neglecting self-mixing).

\section{B.1 $\psi^{2} \varphi^{2} D$ operators}

The matching in $\mathrm{G}_{\mathrm{SM}}$ models at the scale $\mu_{M}$ of order of the VLQ mass yields nonvanishing contributions for

$$
\begin{aligned}
D: & {\left[\mathcal{C}_{H q}^{(1)}\right]_{i j}=\left[\mathcal{C}_{H q}^{(3)}\right]_{i j}=-\frac{1}{4} \frac{\lambda_{i}^{*} \lambda_{j}}{M^{2}}, } \\
T_{d}: & {\left[\mathcal{C}_{H q}^{(1)}\right]_{i j}=-3\left[\mathcal{C}_{H q}^{(3)}\right]_{i j}=-\frac{3}{8} \frac{\lambda_{i}^{*} \lambda_{j}}{M^{2}}, } \\
T_{u}: & {\left[\mathcal{C}_{H q}^{(1)}\right]_{i j}=3\left[\mathcal{C}_{H q}^{(3)}\right]_{i j}=\frac{3}{8} \frac{\lambda_{i}^{*} \lambda_{j}}{M^{2}} } \\
Q_{d}: & {\left[\mathcal{C}_{H d}\right]_{i j}=-\frac{1}{2} \frac{\lambda_{i} \lambda_{j}^{*}}{M^{2}}, } \\
Q_{V}: & {\left[\mathcal{C}_{H d}\right]_{i j}=\frac{1}{2} \frac{\lambda_{i}^{V_{d}} \lambda_{j}^{V_{d}^{*}}}{M^{2}}, \quad\left[\mathcal{C}_{H u}\right]_{i j}=-\frac{1}{2} \frac{\lambda_{i}^{V_{u}} \lambda_{j}^{V_{u} *}}{M^{2}}, \quad\left[\mathcal{C}_{H u d}\right]_{i j}=\frac{\lambda_{i}^{V_{u}} \lambda_{j}^{V_{d^{*}}}}{M^{2}} }
\end{aligned}
$$

in agreement with [3], and analogously for $\mathrm{G}_{\mathrm{SM}}^{\prime}(\Phi)$ models with $H \rightarrow \Phi$. The matching of $\mathrm{G}_{\mathrm{SM}}^{\prime}(S)$ models for VLQs $D$ and $Q_{V}$ yields nonvanishing Wilson coefficients

$$
D: \quad\left[\mathcal{C}_{S d}\right]_{i j}=-\frac{1}{2} \frac{\lambda_{i} \lambda_{j}^{*}}{M^{2}}, \quad Q_{V}: \quad\left[\mathcal{C}_{S q}\right]_{i j}=-\frac{1}{2} \frac{\lambda_{i}^{*} \lambda_{j}}{M^{2}} .
$$

The flavour-changing $Z$ and $Z^{\prime}$ couplings (3.7) and (3.8) after spontaneous symmetry breaking are given in terms of the Wilson coefficients at the scale $\mu_{\mathrm{EW}}$. In the case of 
$\mathrm{G}_{\mathrm{SM}}$-models, the tree-level calculation of the process $\bar{f}_{i} f_{j} Z_{\mu}$ from $\mathrm{G}_{\mathrm{SM}}$-EFT (3.2) yields

$$
\begin{aligned}
\Delta_{L}^{u_{i} u_{j}}(Z) & =\mathcal{F}_{H}\left[\left[\mathcal{C}_{H q}^{(1)}\right]_{, i j}-\left[\mathcal{C}_{H q}^{(3)}\right]_{, i j}\right], & \Delta_{L}^{d_{i} d_{j}}(Z) & =\mathcal{F}_{H}\left[\left[\mathcal{C}_{H q}^{(1)}\right]_{, i j}+\left[\mathcal{C}_{H q}^{(3)}\right]_{, i j}\right], \\
\Delta_{R}^{u_{i} u_{j}}(Z) & =\mathcal{F}_{H}\left[\mathcal{C}_{H u}\right]_{, i j}, & \Delta_{R}^{d_{i} d_{j}}(Z) & =\mathcal{F}_{H}\left[\mathcal{C}_{H d}\right]_{, i j},
\end{aligned}
$$

with $\mathcal{F}_{H} \equiv-2 M_{Z}^{2} / g_{Z}$ and generation indices $i, j=1,2,3$. The variant of $\mathrm{G}_{\mathrm{SM}}^{\prime}(S)$-models with the scalar sector of $S$ and $H$ generates only non-zero couplings to $Z^{\prime}$. We find for $\mathrm{G}_{\mathrm{SM}}^{\prime}(S)$-models

$$
\Delta_{L}^{u_{i} u_{j}, d_{i} d_{j}}\left(Z^{\prime}\right)=\mathcal{F}_{S}\left[\mathcal{C}_{S q}\right]_{, i j}, \quad \Delta_{R}^{u_{i} u_{j}}\left(Z^{\prime}\right)=\mathcal{F}_{S}\left[\mathcal{C}_{S u}\right]_{, i j}, \quad \Delta_{R}^{d_{i} d_{j}}\left(Z^{\prime}\right)=\mathcal{F}_{S}\left[\mathcal{C}_{S d}\right]_{, i j},
$$

with the EFT-coefficients $\mathcal{C}_{i}$ given in (B.1) and $\mathcal{F}_{S} \equiv m_{Z^{\prime}}^{2} /\left(g^{\prime} X\right)$. The variant of $\mathrm{G}_{\mathrm{SM}}^{\prime}(\Phi)$ models with the scalar sector of $S, H$ and $\Phi$ generates non-zero couplings to $Z^{\prime}$ and $Z$. The results for $\mathrm{G}_{\mathrm{SM}}^{\prime}(\Phi)$ models are similar to $\mathrm{G}_{\mathrm{SM}}$ models, with the difference that they involve $Z-Z^{\prime}$ mixings:

$$
\begin{array}{ll}
\Delta_{L}^{u_{i} u_{j}}(V)=\mathcal{F}_{\Phi}(V)\left[\left[\mathcal{C}_{\Phi q}^{(1)}\right]_{, i j}-\left[\mathcal{C}_{\Phi q}^{(3)}\right]_{, i j}\right], & \Delta_{L}^{d_{i} d_{j}}(V)=\mathcal{F}_{\Phi}(V)\left[\left[\mathcal{C}_{\Phi q}^{(1)}\right]_{, i j}+\left[\mathcal{C}_{\Phi q}^{(3)}\right]_{, i j}\right], \\
\Delta_{R}^{u_{i} u_{j}}(V)=\mathcal{F}_{\Phi}(V)\left[\mathcal{C}_{\Phi u}\right]_{, i j}, & \Delta_{R}^{d_{i} d_{j}}(V)=\mathcal{F}_{\Phi}(V)\left[\mathcal{C}_{\Phi d}\right]_{, i j},
\end{array}
$$

where $V=Z, Z^{\prime}$ and

$$
\begin{aligned}
\mathcal{F}_{\Phi}(Z) & \equiv-2 \frac{M_{Z}^{2}}{g_{Z}} c_{\beta}^{2}\left[\cos \xi_{Z Z^{\prime}}-r^{\prime} \sin \xi_{Z Z^{\prime}}\right] \\
\mathcal{F}_{\Phi}\left(Z^{\prime}\right) & \equiv+2 \frac{M_{Z}^{2}}{g_{Z}} c_{\beta}^{2}\left[\sin \xi_{Z Z^{\prime}}+r^{\prime} \cos \xi_{Z Z^{\prime}}\right]
\end{aligned}
$$

\section{B.2 $\psi^{2} \varphi^{3}$ operators}

We define the SM Yukawa couplings of quarks as in [26]

$$
-\mathcal{L}_{\text {Yuk }}=\bar{q}_{L} Y_{d} H d_{R}+\bar{q}_{L} Y_{u} \widetilde{H} u_{R}+\text { h.c.. }
$$

Nonvanishing Wilson coefficients are generated also for $\psi^{2} \varphi^{3}$ operators (see table 2 for definitions) as a consequence of the application of equations of motion (EOM) in the treelevel decoupling of VLQs in section 3.1. Due to the application of EOMs, these Wilson coefficients scale with the corresponding Yukawa coupling as

$$
\begin{aligned}
& {\left[\mathcal{C}_{u H}\right]_{i j}=\left[Y_{u} \mathcal{C}_{H u D}^{\dagger}+\left(\mathcal{C}_{H q D}^{(1)}-\mathcal{C}_{H q D}^{(3)}\right) Y_{u}\right]_{i j},} \\
& {\left[\mathcal{C}_{d H}\right]_{i j}=\left[Y_{d} \mathcal{C}_{H d D}^{\dagger}+\left(\mathcal{C}_{H q D}^{(1)}+\mathcal{C}_{H q D}^{(3)}\right) Y_{d}\right]_{i j}}
\end{aligned}
$$

Note the matrix multiplications w.r.t. the generation indices of $Y_{u, d}$ with the respective coefficients $\mathcal{C}_{H \psi D}$ inside the brackets. 
The tree-level matching in $\mathrm{G}_{\mathrm{SM}}$-models gives nonvanishing contributions at $\mu_{M}$ to

$$
\begin{array}{rlrlrl}
D: & \left.\mathcal{C}_{H q D}^{(1)}+\mathcal{C}_{H q D}^{(3)}\right]_{i j} & =\frac{1}{2} \frac{\lambda_{i}^{*} \lambda_{j}}{M^{2}}, & & {\left[\mathcal{C}_{H q D}^{(1)}-\mathcal{C}_{H q D}^{(3)}\right]_{i j}} & =0, \\
T_{d}: & \left.\mathcal{C}_{H q D}^{(1)}+\mathcal{C}_{H q D}^{(3)}\right]_{i j} & =\frac{1}{4} \frac{\lambda_{i}^{*} \lambda_{j}}{M^{2}}, & {\left[\mathcal{C}_{H q D}^{(1)}-\mathcal{C}_{H q D}^{(3)}\right]_{i j}} & =\frac{1}{2} \frac{\lambda_{i}^{*} \lambda_{j}}{M^{2}}, \\
T_{u}: & {\left[\mathcal{C}_{H q D}^{(1)}+\mathcal{C}_{H q D}^{(3)}\right]_{i j}} & =\frac{1}{2} \frac{\lambda_{i}^{*} \lambda_{j}}{M^{2}}, & {\left[\mathcal{C}_{H q D}^{(1)}-\mathcal{C}_{H q D}^{(3)}\right]_{i j}} & =\frac{1}{4} \frac{\lambda_{i}^{*} \lambda_{j}}{M^{2}}, \\
Q_{d}: & {\left[\mathcal{C}_{H d D}\right]_{i j}} & =\frac{1}{2} \frac{\lambda_{i} \lambda_{j}^{*}}{M^{2}}, & & \\
Q_{V}: & {\left[\mathcal{C}_{H d D}\right]_{i j}} & =\frac{1}{2} \frac{\lambda_{i}^{V_{d}} \lambda_{j}^{V_{d}}}{M^{2}}, & {\left[\mathcal{C}_{H u D}\right]_{i j}} & =\frac{1}{2} \frac{\lambda_{i}^{V_{u}} \lambda_{j}^{V_{u}}}{M^{2}},
\end{array}
$$

in agreement with [3]. Analogous Wilson coefficients in $\mathrm{G}_{\mathrm{SM}}^{\prime}(\Phi)$ are found by $H \rightarrow \Phi$.

In $\mathrm{G}_{\mathrm{SM}}^{\prime}(S)$ models analogous relations

$$
\left[\mathcal{C}_{u S}\right]_{i j}=\left[Y_{u} \mathcal{C}_{S u D}^{\dagger}+\mathcal{C}_{S q D} Y_{u}\right]_{i j}, \quad\left[\mathcal{C}_{d S}\right]_{i j}=\left[Y_{d} \mathcal{C}_{S d D}^{\dagger}+\mathcal{C}_{S q D} Y_{d}\right]_{i j}
$$

hold with nonvanishing

$$
D: \quad\left[\mathcal{C}_{S d D}\right]_{i j}=\frac{\lambda_{i} \lambda_{j}^{*}}{M^{2}}, \quad Q_{V}: \quad\left[\mathcal{C}_{S q D}\right]_{i j}=\frac{\lambda_{i}^{*} \lambda_{j}}{M^{2}}
$$

\section{B.3 Top-Yukawa RG effects}

This appendix collects the ADM entries of the $\mathrm{G}_{\mathrm{SM}}$-EFT proportional to the up-type quark Yukawa coupling $Y_{u}$ from [28], i.e. neglecting contributions from $Y_{d, e}$. We list them only for operators that receive leading logarithmic contributions at the scale $\mu_{\mathrm{EW}}$ from the initial Wilson coefficients at the scale $\mu_{M}$ of $\psi^{2} H^{2} D$ and $\psi^{2} H^{3}$ operators in the 1stLLA via direct mixing, see footnote 3 . For convenience of the reader we keep here also $\mathcal{C}_{H u}$ and $\mathcal{C}_{\mathrm{Hud}}$, which are absent in the VLQ models $D, T_{u}, T_{d}, Q_{d}$, but contribute in $Q_{V}$ for $\lambda^{V_{u}} \neq 0$.

The $H^{6}$-operator $\mathcal{O}_{H}=\left(H^{\dagger} H\right)^{3}$ receives direct leading logarithmic contributions ${ }^{16}$

$$
\dot{\mathcal{C}}_{H} \equiv(4 \pi)^{2} \mu \frac{\mathrm{d} \mathcal{C}_{H}}{\mathrm{~d} \mu}=-12 \operatorname{Tr}\left[\mathcal{C}_{u H} Y_{u}^{\dagger} Y_{u} Y_{u}^{\dagger}+Y_{u} Y_{u}^{\dagger} Y_{u} \mathcal{C}_{u H}^{\dagger}\right]
$$

via $\mathcal{C}_{u H} \neq 0$ in models VLQ $=T_{u}, T_{d}$. The Wilson coefficent $\mathcal{C}_{H}$ changes the Higgs potential and leads to a shift of the VEV [29].

The $H^{4} D^{2}$-operators $\mathcal{O}_{H \square}=\left(H^{\dagger} H\right) \square\left(H^{\dagger} H\right)$ and $\mathcal{O}_{H D}=\left(H^{\dagger} \mathcal{D}_{\mu} H\right)^{*}\left(H^{\dagger} \mathcal{D}^{\mu} H\right)$ receive leading logarithmic contributions in LH models $\mathrm{VLQ}=D, T_{u}, T_{d}$ via $\mathcal{C}_{H q}^{(1,3)}$ :

$$
\begin{aligned}
& \dot{\mathcal{C}}_{H \square}=6 \operatorname{Tr}\left[\left(\mathcal{C}_{H q}^{(1)}-3 \mathcal{C}_{H q}^{(3)}\right) Y_{u} Y_{u}^{\dagger}-\mathcal{C}_{H u} Y_{u}^{\dagger} Y_{u}\right], \\
& \dot{\mathcal{C}}_{H D}=24 \operatorname{Tr}\left[\mathcal{C}_{H q}^{(1)} Y_{u} Y_{u}^{\dagger}-\mathcal{C}_{H u} Y_{u}^{\dagger} Y_{u}\right] .
\end{aligned}
$$

Their Wilson coefficients contribute to the Higgs-boson mass and the electroweak precision observable $T=-2 \pi v^{2}\left(g_{1}^{-2}+g_{2}^{-2}\right) \mathcal{C}_{H D}[29]$.

\footnotetext{
${ }^{16}$ Note that if the generation indices are not given explicitly on Yukawa couplings and Wilson coefficients then a matrix multiplication is implied.
} 
The $\psi^{2} H^{3}$-operators (see table 2)

$$
\begin{aligned}
\dot{\mathcal{C}}_{u H}= & -12 \operatorname{Tr}\left[\mathcal{C}_{H q}^{(3)} Y_{u} Y_{u}^{\dagger}\right] Y_{u}-2 \mathcal{C}_{H q}^{(1)} Y_{u} Y_{u}^{\dagger} Y_{u}+2 Y_{u} Y_{u}^{\dagger} Y_{u} \mathcal{C}_{H u} \\
& +6 \operatorname{Tr}\left[\mathcal{C}_{u H} Y_{u}^{\dagger}\right] Y_{u}+9 \operatorname{Tr}\left[Y_{u} Y_{u}^{\dagger}\right] \mathcal{C}_{u H}+5 \mathcal{C}_{u H} Y_{u}^{\dagger} Y_{u}+\frac{11}{2} Y_{u} Y_{u}^{\dagger} \mathcal{C}_{u H} \\
\dot{\mathcal{C}}_{d H}= & -12 \operatorname{Tr}\left[\mathcal{C}_{H q}^{(3)} Y_{u} Y_{u}^{\dagger}\right] Y_{d}+6 \mathcal{C}_{H q}^{(3)} Y_{u} Y_{u}^{\dagger} Y_{d}-2 Y_{u} Y_{u}^{\dagger} Y_{u} \mathcal{C}_{H u d} \\
& +6 \operatorname{Tr}\left[Y_{u} \mathcal{C}_{u H}^{\dagger}\right] Y_{d}-2 Y_{u} \mathcal{C}_{u H}^{\dagger} Y_{d}-\mathcal{C}_{u H} Y_{u}^{\dagger} Y_{d}+9 \operatorname{Tr}\left[Y_{u} Y_{u}^{\dagger}\right] \mathcal{C}_{d H}-\frac{3}{2} Y_{u} Y_{u}^{\dagger} \mathcal{C}_{d H} \\
\dot{\mathcal{C}}_{e H}= & -12 \operatorname{Tr}\left[\mathcal{C}_{H q}^{(3)} Y_{u} Y_{u}^{\dagger}\right] Y_{e}+6 \operatorname{Tr}\left[Y_{u} \mathcal{C}_{u H}^{\dagger}\right] Y_{e}
\end{aligned}
$$

have self-mixing for $\mathcal{C}_{u H, d H}$, and $\mathcal{C}_{u H}$ mixes also into $\mathcal{C}_{d H, e H}$. They receive also contributions from $\mathcal{C}_{\psi^{2} H^{2} D}$. The $\mathcal{C}_{\psi^{2} H^{3}}$ enter fermion-mass matrices (3.4) and lead also to fermion-Higgs couplings that are in general flavour-off-diagonal.

The $\psi^{2} H^{2} D$-operators (see table 2)

$$
\begin{aligned}
\dot{\mathcal{C}}_{H q}^{(1)}= & 6 \operatorname{Tr}\left[Y_{u} Y_{u}^{\dagger}\right] \mathcal{C}_{H q}^{(1)}+2\left(Y_{u} Y_{u}^{\dagger} \mathcal{C}_{H q}^{(1)}+\mathcal{C}_{H q}^{(1)} Y_{u} Y_{u}^{\dagger}\right) \\
& -\frac{9}{2}\left(Y_{u} Y_{u}^{\dagger} \mathcal{C}_{H q}^{(3)}+\mathcal{C}_{H q}^{(3)} Y_{u} Y_{u}^{\dagger}\right)-Y_{u} \mathcal{C}_{H u} Y_{u}^{\dagger} \\
\dot{\mathcal{C}}_{H q}^{(3)}= & 6 \operatorname{Tr}\left[Y_{u} Y_{u}^{\dagger}\right] \mathcal{C}_{H q}^{(3)}+Y_{u} Y_{u}^{\dagger} \mathcal{C}_{H q}^{(3)}+\mathcal{C}_{H q}^{(3)} Y_{u} Y_{u}^{\dagger}-\frac{3}{2}\left(Y_{u} Y_{u}^{\dagger} \mathcal{C}_{H q}^{(1)}+\mathcal{C}_{H q}^{(1)} Y_{u} Y_{u}^{\dagger}\right) \\
\dot{\mathcal{C}}_{H d}= & 6 \operatorname{Tr}\left[Y_{u} Y_{u}^{\dagger}\right] \mathcal{C}_{H d}, \\
\dot{\mathcal{C}}_{H u}= & -2 Y_{u}^{\dagger} \mathcal{C}_{H q}^{(1)} Y_{u}+6 \operatorname{Tr}\left[Y_{u} Y_{u}^{\dagger}\right] \mathcal{C}_{H u}+4\left(Y_{u}^{\dagger} Y_{u} \mathcal{C}_{H u}+\mathcal{C}_{H u} Y_{u}^{\dagger} Y_{u}\right) \\
\dot{\mathcal{C}}_{H u d}= & 6 \operatorname{Tr}\left[Y_{u} Y_{u}^{\dagger}\right] \mathcal{C}_{H u d}+3 Y_{u}^{\dagger} Y_{u} \mathcal{C}_{H u d}
\end{aligned}
$$

show a mixing pattern among $\mathcal{C}_{H q}^{(1,3)}$ as well as $\mathcal{C}_{H q}^{(1)}$ and $\mathcal{C}_{H u}$. The latter implies that the LH scenarios $D, T_{u}, T_{d}$ will generate via mixing also a $\mathrm{RH}$ coupling $\mathcal{C}_{H u}$ via $\mathcal{C}_{H q}^{(1)}$, which is however a one-loop effect compared to the effects of $\mathcal{C}_{H q}^{(1)}$. Both $\mathcal{C}_{H d}$ and $\mathcal{C}_{H u d}$ have only self-mixing.

In the case of $\psi^{4}$-operators there are $(\bar{L} L)(\bar{L} L)$ operators

$$
\begin{aligned}
& {\left[\dot{\mathcal{C}}_{q q}^{(1)}\right]_{i j k l}=+\frac{1}{2}\left(\left[Y_{u} Y_{u}^{\dagger}\right]_{i j}\left[\mathcal{C}_{H q}^{(1)}\right]_{k l}+\left[\mathcal{C}_{H q}^{(1)}\right]_{i j}\left[Y_{u} Y_{u}^{\dagger}\right]_{k l}\right),} \\
& {\left[\dot{\mathcal{C}}_{q q}^{(3)}\right]_{i j k l}=-\frac{1}{2}\left(\left[Y_{u} Y_{u}^{\dagger}\right]_{i j}\left[\mathcal{C}_{H q}^{(3)}\right]_{k l}+\left[\mathcal{C}_{H q}^{(3)}\right]_{i j}\left[Y_{u} Y_{u}^{\dagger}\right]_{k l}\right),}
\end{aligned}
$$

the $(\bar{L} L)(\bar{R} R)$ operators

$$
\begin{aligned}
{\left[\dot{\mathcal{C}}_{q u}^{(1)}\right]_{i j k l} } & =\left[Y_{u} Y_{u}^{\dagger}\right]_{i j}\left[\mathcal{C}_{H u}\right]_{k l}-2\left[\mathcal{C}_{H q}^{(1)}\right]_{i j}\left[Y_{u}^{\dagger} Y_{u}\right]_{k l} \\
{\left[\dot{\mathcal{C}}_{q d}^{(1)}\right]_{i j k l} } & =\left[Y_{u} Y_{u}^{\dagger}\right]_{i j}\left[\mathcal{C}_{H d}\right]_{k l}
\end{aligned}
$$

and the $(\bar{R} R)(\bar{R} R)$ operators

$$
\begin{aligned}
{\left[\dot{\mathcal{C}}_{u u}\right]_{i j k l} } & =-\left[Y_{u}^{\dagger} Y_{u}\right]_{i j}\left[\mathcal{C}_{H u}\right]_{k l}-\left[\mathcal{C}_{H u}\right]_{i j}\left[Y_{u}^{\dagger} Y_{u}\right]_{k l}, \\
{\left[\dot{\mathcal{C}}_{u d}^{(1)}\right]_{i j k l} } & =-2\left[Y_{u}^{\dagger} Y_{u}\right]_{i j}\left[\mathcal{C}_{H d}\right]_{k l},
\end{aligned}
$$


of which the ones relevant for $|\Delta F|=2$ are given in (3.14) and (3.15). Hence there are two additional operators $\left[\mathcal{O}_{u d}^{(1)}\right]_{i j k l}=\left[\bar{u}_{R}^{i} \gamma_{\mu} u_{R}^{j}\right]\left[\bar{d}_{R}^{k} \gamma^{\mu} d_{R}^{l}\right]$ and $\left[\mathcal{O}_{q u}^{(1)}\right]_{i j k l}=\left[\bar{q}_{L}^{i} \gamma_{\mu} q_{L}^{j}\right]\left[\bar{u}_{R}^{k} \gamma^{\mu} u_{R}^{l}\right]$ under the assumption $\mathcal{C}_{\mathrm{Hu}}=0$.

\section{Master formulae for $K$ and $B$ decays}

\section{C.1 $|\Delta F|=2$}

The effective Lagrangian for neutral meson mixing in the down-type quark sector $\left(d_{j} \bar{d}_{i} \rightarrow\right.$ $\bar{d}_{j} d_{i}$ with $i \neq j$ ) can be written as [34]

$$
\mathcal{H}_{\Delta F=2}^{i j}=\mathcal{N}_{i j} \sum_{a} C_{a}^{i j} O_{a}^{i j}+\text { h.c. }
$$

where the normalisation factor and the CKM combinations are

$$
\mathcal{N}_{i j}=\frac{G_{F}^{2}}{4 \pi^{2}} M_{W}^{2}\left(\lambda_{i j}^{(t)}\right)^{2}
$$

with $i j=s d$ for kaon mixing and $i j=b d, b s$ for $B_{d}$ and $B_{s}$ mixing, respectively. The set of operators consists out of $(5+3)=8$ operators [34],

$$
\begin{array}{rlrl}
O_{\mathrm{VLL}}^{i j} & =\left[\bar{d}_{i} \gamma_{\mu} P_{L} d_{j}\right]\left[\bar{d}_{i} \gamma^{\mu} P_{L} d_{j}\right], & & \\
O_{\mathrm{LR}, 1}^{i j} & =\left[\bar{d}_{i} \gamma_{\mu} P_{L} d_{j}\right]\left[\bar{d}_{i} \gamma^{\mu} P_{R} d_{j}\right], & O_{\mathrm{LR}, 2}^{i j}=\left[\bar{d}_{i} P_{L} d_{j}\right]\left[\bar{d}_{i} P_{R} d_{j}\right], \\
O_{\mathrm{SLL}, 1}^{i j} & =\left[\bar{d}_{i} P_{L} d_{j}\right]\left[\bar{d}_{i} P_{L} d_{j}\right], & O_{\mathrm{SLL}, 2}^{i j}=-\left[\bar{d}_{i} \sigma_{\mu \nu} P_{L} d_{j}\right]\left[\bar{d}_{i} \sigma^{\mu \nu} P_{L} d_{j}\right],
\end{array}
$$

which are built out of colour-singlet currents $\left[\bar{d}_{i}^{\alpha} \ldots d_{j}^{\alpha}\right]\left[\bar{d}_{i}^{\beta} \ldots d_{j}^{\beta}\right]$, where $\alpha, \beta$ denote colour indices. The chirality-flipped sectors VRR and SRR are obtained from interchanging $P_{L} \leftrightarrow$ $P_{R}$ in VLL and SLL. Note that the minus sign in $Q_{\mathrm{SLL}, 2}$ arises from different definitions of $\tilde{\sigma}_{\mu \nu} \equiv\left[\gamma_{\mu}, \gamma_{\nu}\right] / 2$ in ref. [34] w.r.t. $\sigma_{\mu \nu}=i \tilde{\sigma}_{\mu \nu}$ used here. The ADM's of the 5 distinct sectors (VLL, SLL, LR, VRR, SRR) have been calculated in refs. [33, 34] at NLO in QCD, and numerical solutions are given in ref. [97]. The NLO ADM's are also available for an alternative basis [98] with colour octet operators $Q_{\mathrm{SLL}, 2}=\left[\bar{d}_{i}^{\alpha} P_{L} d_{j}^{\beta}\right]\left[\bar{d}_{i}^{\beta} P_{L} d_{j}^{\alpha}\right]$ and analogous $Q_{\mathrm{SRR}, 2}$.

In the SM only

$$
\left.C_{\mathrm{VLL}}^{i j}\left(\mu_{\mathrm{EW}}\right)\right|_{\mathrm{SM}}=S_{0}\left(x_{t}\right), \quad S_{0}(x)=\frac{x\left(4-11 x+x^{2}\right)}{4(x-1)^{2}}+\frac{3 x^{3} \ln x}{2(x-1)^{3}}
$$

is non-zero at the scale $\mu_{\mathrm{EW}}$, depending on the ratio $x_{t} \equiv m_{t}^{2} / M_{W}^{2}$ of the top-quark and $W$-boson masses.

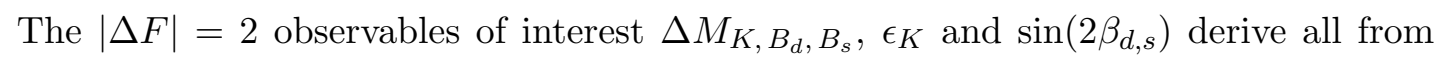
the complex-valued off-diagonal elements $M_{12}^{i j}$ of the mass-mixing matrices of the neutral mesons [99, 100]. For the latter we use the full higher-order SM expressions in combination with the LO new physics contributions. In particular for $M_{12}^{d s}$, we make use of NLO and in part NNLO QCD corrections $\eta_{c c, t t, c t}$ collected in table 13 and for the hadronic matrix element of $|\Delta S|=2$ operators the value of $\hat{B}_{K}$. Concerning $|\Delta B|=2$, we include the NLO QCD corrections $\eta_{B}$ to the SM and use for the hadronic matrix elements the latest results for $F_{B_{d, s}} \sqrt{\hat{B}_{B_{d, s}}}$ [61]. The hadronic matrix elements of $|\Delta S, B|=2$ of left-right operators are given in table 14 . 


\section{C.2 $\quad d_{j} \rightarrow d_{i} \nu \bar{\nu}$}

The effective Lagrangian for $d_{j} \rightarrow d_{i} \nu \bar{\nu}(i \neq j)$ is adopted from ref. [91],

$$
\mathcal{L}_{d \rightarrow d \nu \bar{\nu}}=\frac{4 G_{F}}{\sqrt{2}} \frac{\alpha_{e}}{4 \pi} \lambda_{i j}^{(t)} \sum_{a} \sum_{\nu} C_{a}^{i j, \nu} O_{a}^{i j, \nu}+\text { h.c. },
$$

where the sums extend over $a=\{L, R\}$ and neutrino flavour $\nu=\{e, \mu, \tau\}$

$$
O_{L(R)}^{i j, \nu}=\left[\bar{d}_{i} \gamma_{\mu} P_{L(R)} d_{j}\right]\left[\bar{\nu} \gamma^{\mu}\left(1-\gamma_{5}\right) \nu\right] .
$$

In the SM only

$$
\left.C_{L}^{i j, \nu}\right|_{\mathrm{SM}}=\frac{4 B-C}{s_{W}^{2}} \equiv-\frac{X_{0}}{s_{W}^{2}}
$$

has non-vanishing contribution at the scale $\mu_{\mathrm{EW}}$, whereas $C_{R}^{\nu}=0$. The functions $B$ and $C$ depend on the ratio $x_{t} \equiv m_{t}^{2} / M_{W}^{2}$ of the top-quark and $W$-boson masses and enter as the gauge-independent linear combination $X_{0}\left(x_{t}\right) \equiv C\left(x_{t}\right)-4 B\left(x_{t}\right)[101,102]$,

$$
X_{0}(x)=\frac{x}{8}\left(\frac{x+2}{x-1}+\frac{3 x-6}{(x-1)^{2}} \ln x\right) .
$$

It is given by

$$
X_{0} \rightarrow X_{L}^{\mathrm{SM}}=1.481 \pm 0.009,
$$

when including higher order QCD and electroweak corrections [103-106] as extracted in ref. [107] from original papers.

The theoretical predictions for $b \rightarrow s \nu \bar{\nu}$ observables defined in eq. (6.5) are based on formulae given in ref. [92]. These expressions account for the lepton-non-universal contribution of VLQ's w.r.t. the neutrino flavour in $\mathrm{G}_{\mathrm{SM}}^{\prime}$ models. However, the particular structure of the gauged $\mathrm{U}(1)_{\mathrm{L}_{\mu}-\mathrm{L}_{\tau}}$ (2.1) leads to a cancellation of the numerically leading interference contributions of the SM and new physics [9].

The $\operatorname{Br}\left(K^{+} \rightarrow \pi^{+} \nu \bar{\nu}\right)$ receives in the SM the numerically leading contribution from the "top"-sector, when decoupling heavy degrees of freedom at $\mu_{\mathrm{EW}}$, which yields directly the local $\mathcal{O}_{L}^{s d, \nu}$ operator $(\nu=e, \mu, \tau)$. Further, a non-negligible "charm"-sector arises from double-insertions of hadronic and semi-leptonic $|\Delta S|=1$ operators when decoupling the charm quark at $\mu_{c} \sim m_{c}$, which is enhanced due to the strong CKM hierarchy $\left(\lambda_{s d}^{(t)} \propto\right.$ $\left.\lambda^{5}\right) \ll\left(\lambda_{s d}^{(c)} \propto \lambda^{2}\right)$, where $\lambda=\left|V_{u s}\right|$ is the Cabibbo angle. This is usually expressed in the effective Hamiltonian of the SM as [108]

$$
\mathcal{H}_{\mathrm{eff}}=\mathcal{N} \sum_{\nu}\left[\lambda_{s d}^{(c)} X_{c}^{\nu}+\lambda_{s d}^{(t)} X_{L}^{\mathrm{SM}}\right] \mathcal{O}_{L}^{s d, \nu}
$$

with $\mathcal{N}=G_{F} \alpha_{e} /\left(2 \sqrt{2} \pi s_{W}^{2}\right)$, where $X_{c}^{e}=X_{c}^{\mu} \neq X_{c}^{\tau}$.

The NP contributions in VLQ-models cannot compete with the SM contribution to the tree-level processes entering the "charm"-sector, since they are suppressed by an additional factor $\left(M_{W} / M_{\mathrm{VLQ}}\right)^{2}$. In consequence, NP contributes to the "top"-sector only

$$
X_{L}^{\mathrm{SM}} \quad \rightarrow \quad X_{t}^{\nu}=X_{L}^{\mathrm{SM}}+X_{L}^{s d, \nu}+X_{R}^{s d, \nu} \equiv X_{L}^{\mathrm{SM}}+X_{\mathrm{NP}}^{\nu}
$$


with $X_{L, R}^{s d, \nu}$ given in eq. (4.8), such that the top-sector becomes neutrino-flavour dependent.

The experimental measurement averages over the three neutrino flavours,

$$
\operatorname{Br}\left(K^{+} \rightarrow \pi^{+} \nu \bar{\nu}\right)=\frac{\kappa_{+}\left(1+\Delta_{\mathrm{EM}}\right)}{\lambda^{10}} \frac{1}{3} \sum_{\nu}\left[\operatorname{Im}^{2}\left(\lambda_{s d}^{(t)} X_{t}^{\nu}\right)+\operatorname{Re}^{2}\left(\lambda_{s d}^{(c)} X_{c}^{\nu}+\lambda_{s d}^{(t)} X_{t}^{\nu}\right)\right]
$$

with the assumption that $\lambda_{s d}^{(c)} X_{c}^{\nu}$ is real. The NNLO QCD results of the functions $X_{c}^{\nu}$ [108] together with long distance contributions [109] are combined into

$$
P_{c}=\frac{1}{\lambda^{4}}\left(\frac{2}{3} X_{c}^{e}+\frac{1}{3} X_{c}^{\tau}\right)=\left(\frac{0.2252}{\lambda}\right)^{4}(0.404 \pm 0.024),
$$

where $\lambda=0.2252$ has been used in ref. [107]. The factor

$$
\kappa_{+}=r_{K^{+}} \frac{3 \alpha^{2}\left(M_{Z}\right) \lambda^{8}}{2 \pi^{2} s_{W}^{4}} \operatorname{Br}\left(K \rightarrow \pi e \bar{\nu}_{e}\right)=0.5173(25) \times 10^{-10}\left[\frac{\lambda}{0.225}\right]^{8}
$$

contains the experimental value $\operatorname{Br}\left(K \rightarrow \pi e \bar{\nu}_{e}\right)$ and the isospin correction $r_{K^{+}}$and has been evaluated in ref. [110] (table 2) including various corrections. Further $\Delta_{\mathrm{EM}}=-0.003$ for $E_{\max }^{\gamma} \approx 20 \mathrm{MeV}$ [110]. If one takes into account the different value of $s_{W}^{2}=0.231$ taken in ref. [110] compared to our value in table 13, then $\kappa_{+}=0.5150 \times 10^{-10}(\lambda / 0.225)^{8}$.

The sum (C.12) contains the SM contribution and further the interference of $\mathrm{SM} \times \mathrm{NP}$ and $\mathrm{NP} \times \mathrm{NP}$. Besides $P_{c}$ at NNLO in the SM contribution, the NLO numerical values

$$
X_{c}^{e}=10.05 \times 10^{-4}, \quad X_{c}^{\tau}=6.64 \times 10^{-4},
$$

for $\mu_{c}=1.3 \mathrm{GeV}$ are used for the interference of $\mathrm{SM} \times \mathrm{NP}$.

The branching fraction of $K_{L} \rightarrow \pi^{0} \nu \bar{\nu}$ is obtained again by averaging over the three neutrino flavours

$$
B r\left(K_{L} \rightarrow \pi^{0} \nu \bar{\nu}\right)=\frac{\kappa_{L}}{\lambda^{10}} \frac{1}{3} \sum_{\nu} \operatorname{Im}^{2}\left(\lambda_{s d}^{(t)} X_{t}^{\nu}\right)
$$

with

$$
\kappa_{L}=\kappa_{+} \frac{r_{K_{L}}}{r_{K_{+}}} \frac{\tau_{K_{L}}}{\tau_{K_{+}}}=2.231(13) \times 10^{-10}\left[\frac{\lambda}{0.225}\right]^{8} .
$$

The numerical value is from ref. [110] (table 2) and it decreases to $\kappa_{L}=2.221 \times$ $10^{-10}(\lambda / 0.225)^{8}$ when rescaling with our value of $s_{W}^{2}$.

\section{C.3 $d_{j} \rightarrow d_{i} \ell \bar{\ell}$}

The effective Lagrangian for $d_{j} \rightarrow d_{i} \ell \bar{\ell}(i \neq j)$ is adopted from ref. [111],

$$
\mathcal{L}_{d \rightarrow d e \bar{\ell}}=\frac{4 G_{F}}{\sqrt{2}} \frac{\alpha_{e}}{4 \pi} \lambda_{i j}^{(t)} \sum_{a} \sum_{\ell} C_{a}^{i j, \ell} O_{a}^{i j, \ell}+\text { h.c. }
$$

were the sum over $a$ extends over the $|\Delta F|=1$ operators

$$
O_{9\left(9^{\prime}\right)}^{i j, \ell}=\left[\bar{d}_{i} \gamma_{\mu} P_{L(R)} d_{j}\right]\left[\bar{\ell} \gamma^{\mu} \ell\right], \quad O_{10\left(10^{\prime}\right)}^{i j, \ell}=\left[\bar{d}_{i} \gamma_{\mu} P_{L(R)} d_{j}\right]\left[\bar{\ell} \gamma^{\mu} \gamma_{5} \ell\right],
$$


whereas scalar $O_{\mathrm{S}, \mathrm{P}\left(\mathrm{S}^{\prime}, \mathrm{P}^{\prime}\right)}^{\ell}$ and tensorial operators $O_{\mathrm{T}(\mathrm{T} 5)}^{\ell}$ are not generated in the context of VLQ models. In the SM the only non-zero Wilson coefficients,

$$
\begin{aligned}
& \left.C_{9}^{i j, \ell}\right|_{\mathrm{SM}}=\frac{1}{s_{W}^{2}}\left[\left(1-4 s_{W}^{2}\right) C-B-s_{W}^{2} D\right] \equiv \frac{Y_{0}}{s_{W}^{2}}-4 Z_{0}, \\
& \left.C_{10}^{i j, \ell}\right|_{\mathrm{SM}}=\frac{1}{s_{W}^{2}}(B-C) \equiv-\frac{Y_{0}}{s_{W}^{2}},
\end{aligned}
$$

are lepton-flavour universal and also universal w.r.t. down-type quark transitions, as the CKM elements have been factored out. All other Wilson coefficients vanish at the scale $\mu_{\mathrm{EW}}$. The functions $B, C, D$ depend again on the ratio $x_{t} \equiv m_{t}^{2} / M_{W}^{2}$ of the top-quark and $W$-boson masses and give two gauge-independent combinations $Y_{0}\left(x_{t}\right) \equiv C\left(x_{t}\right)-B\left(x_{t}\right)$ and $Z_{0}\left(x_{t}\right) \equiv C\left(x_{t}\right)+D\left(x_{t}\right) / 4$, that are given in the SM as

$$
\begin{aligned}
& Y_{0}(x)=\frac{x}{8}\left(\frac{x-4}{x-1}+\frac{3 x \ln x}{(x-1)^{2}}\right), \\
& Z_{0}(x)=\frac{18 x^{4}-163 x^{3}+259 x^{2}-108 x}{144(x-1)^{3}}+\frac{32 x^{4}-38 x^{3}-15 x^{2}+18 x}{72(x-1)^{4}} \ln x-\frac{1}{9} \ln x .
\end{aligned}
$$

In the predictions of $\operatorname{Br}\left(B_{d, s} \rightarrow \mu \bar{\mu}\right)$ and the mass-eigenstate rate asymmetry $A_{\Delta \Gamma}\left(B_{d, s} \rightarrow \mu \bar{\mu}\right)$ we include for the SM contribution the NNLO QCD [112] and NLO EW [39] corrections, whereas NP contributions are included at LO. The values of the decay constants $F_{B_{d, s}}$ are collected in table 13 .

The branching fractions $\operatorname{Br}\left(B^{+} \rightarrow\left(\pi^{+}, K^{+}\right) \mu \bar{\mu}\right)$ at high dilepton invariant mass $q^{2}$ are predicted within the framework outlined in refs. [113-115]. We neglect contributions from QCD penguin operators, which have small Wilson coefficients and the NLO QCD corrections to matrix elements of the charged-current operators [116, 117], but include the contributions $\sim V_{u b} V_{u d(s)}^{*}$. The form factors and their uncertainties are adapted from lattice calculations [118, 119] for $B \rightarrow \pi$ and [120] for $B \rightarrow K$ with a summary given in [121]. We add additional relative uncertainties of $15 \%$ for missing NLO QCD corrections and $10 \%$ for possible duality violation [114] in quadrature.

The predictions for observables of $B \rightarrow K^{*} \mu \bar{\mu}$ are based on refs. [89] and [122] for lowand high- $q^{2}$ regions, respectively. The corresponding results for $B \rightarrow K^{*}$ form factors in the two regions are from the LCSR calculation [123] and the lattice calculations [124, 125].

The measurement of $\operatorname{Br}\left(K_{L} \rightarrow \mu \bar{\mu}\right)$ provides important constraints on its shortdistance (SD) contributions, despite the dominating long-distance (LD) contributions inducing uncertainties that are not entirely under theoretical control. In particular there is the issue of the sign of the interference of the SD part $\chi_{\mathrm{SD}}$ of the decay amplitude of $K_{L} \rightarrow \mu \bar{\mu}$ with the LD parts. Allowing for both signs implies a conservative bound $\left|\chi_{\mathrm{SD}}\right| \leq 3.1$ [74]. Relying on predictions of this sign based on the quite general assumptions stated in $[74,126,127]$ one finds $-3.1 \leq \chi_{\mathrm{SD}} \leq 1.7$ which we employ in most of this work. Note, however, that a different sign is found ${ }^{17}$ in $[126,128]$, implying $-1.7 \leq \chi_{\mathrm{SD}} \leq 3.1$. In light of this situation, we comment on the impact of the more conservative choice where appropriate, which includes both sign choices.

\footnotetext{
${ }^{17}$ We thank G. D'Ambrosio and J-M. Gérard for the discussion on this point.
} 


\begin{tabular}{|r|rrr|r|}
\hline$a$ & $p_{a}^{(0)}$ & $p_{a}^{(6)}$ & $p_{a}^{(8)}$ & $P_{a}$ \\
\hline 3 & 7.45 & -3.40 & -3.50 & 2.85 \\
5 & 1.70 & 30.62 & -18.74 & 4.91 \\
7 & -102.02 & -1.32 & 2040.38 & 1447.91 \\
9 & 36.72 & 4.42 & -21.28 & 23.06 \\
\hline
\end{tabular}

Table 12. Values of the coefficients entering the semi-numerical formula of $\varepsilon^{\prime} / \varepsilon$ in eq. (C.29). The last column gives $P_{a}$ for $B_{6}^{(1 / 2)}=0.57$ and $B_{8}^{(3 / 2)}=0.76$.

\section{C.4 $\quad d_{j} \rightarrow d_{i} q \bar{q}$ and $\varepsilon^{\prime} / \varepsilon$}

The effective Lagrangian for $d_{j} \rightarrow d_{i} q \bar{q}(i \neq j)$ is adopted from ref. [129], where the definition of the operators can be found and here we restrict ourselves to $\bar{s} \rightarrow \bar{d}$, i.e. $i j=s d$. At the scale $\mu_{\mathrm{EW}}\left(N_{f}=5\right)$ it reads

$$
\begin{aligned}
\mathcal{L}_{d \rightarrow d q \bar{q}}=-\frac{G_{F}}{\sqrt{2}} \lambda_{s d}^{(u)}\{ & (1-\tau)\left[z_{1}\left(O_{1}-O_{1}^{c}\right)+z_{2}\left(O_{2}-O_{2}^{c}\right)\right] \\
& \left.+\sum_{a=3}^{10}\left(\tau v_{a}+v_{a}^{\mathrm{NP}}\right) O_{a}+\sum_{a=3}^{10} v_{a}^{\prime} O_{a}^{\prime}\right\}+ \text { h.c. }
\end{aligned}
$$

where $O_{1,2}^{(c)}$ denote current-current operators. The sum over $a$ extends over the QCD- and EW-penguin operators and we included their chirality-flipped counterparts $O_{a}^{\prime}=O_{a}\left[\gamma_{5} \rightarrow\right.$ $-\gamma_{5}$ ]. Thereby we assume that VLQ contributions to other operators are strongly suppressed. The Wilson coefficients are denoted as $z_{a}, v_{a}^{(\mathrm{NP})}$ and $v_{a}^{\prime}$, taken at the scale $\mu_{\mathrm{EW}}$. For the SM-part, CKM unitarity was used,

$$
\tau \equiv \lambda_{s d}^{(u)} / \lambda_{s d}^{(t)},
$$

and we introduced a new physics contribution $v_{a}^{\mathrm{NP}}$ as shown above, which is related to the VLQ-contribution (4.13) as

$$
v_{a}^{\mathrm{NP}}=C_{a}^{s d}, \quad v_{a}^{\prime}=C_{a^{\prime}}^{s d} .
$$

The RG evolution at NLO in QCD and QED leads to the effective Hamiltonian at a scale $\mu \lesssim \mu_{c} \sim m_{c}\left(N_{f}=3\right)$

$$
\mathcal{H}_{d \rightarrow d q \bar{q}}=\frac{G_{F}}{\sqrt{2}} \lambda_{s d}^{(u)}\left\{z_{1} O_{1}+z_{2} O_{2}+\sum_{a=3}^{10}\left[z_{a}+\tau y_{a}+v_{a}^{\mathrm{NP}}\right] O_{a}+\sum_{a=3}^{10} v_{a}^{\prime} O_{a}^{\prime}\right\}+\text { h.c. }
$$

after decoupling of $b$ - and $c$-quarks at scales $\mu_{b, c}$ [129], where $y_{a} \equiv v_{a}-z_{a}$ and all Wilson coefficients are at the scale $\mu$.

The contributions of new physics can then be accounted for in $\varepsilon^{\prime} / \varepsilon$ by the replacement

$$
y_{a}(\mu) \rightarrow y_{a}(\mu)+\frac{v_{a}^{\mathrm{NP}}(\mu)-v_{a}^{\prime}(\mu)}{\tau},
$$


where the minus sign is due to $\left\langle(\pi \pi)_{I}\left|O_{a}\right| K\right\rangle=-\left\langle(\pi \pi)_{I}\left|O_{a}^{\prime}\right| K\right\rangle$ for the pseudo-scalar pions in the final state [130]. For the readers convenience we provide a semi-numerical formula for $\varepsilon^{\prime} / \varepsilon$ with initial conditions of Wilson coefficients from new physics in QCD- and EWpenguins $a=3^{(\prime)}, 5^{(\prime)}, 7^{(\prime)}, 9^{(\prime)}$ at the electroweak scale $\mu_{\mathrm{EW}}$ :

$$
\frac{\varepsilon^{\prime}}{\varepsilon}=\left[-2.58+24.01 B_{6}^{(1 / 2)}-12.70 B_{8}^{(3 / 2)}\right] \times 10^{-4}+\sum_{a} P_{a} \operatorname{Im}\left(v_{a}^{\mathrm{NP}}-v_{a}^{\prime}\right)\left[\mu_{\mathrm{EW}}\right] .
$$

The coefficients are

$$
P_{a}=p_{a}^{(0)}+p_{a}^{(6)} B_{6}^{(1 / 2)}+p_{a}^{(8)} B_{8}^{(3 / 2)}
$$

with $p_{a}^{(n)}$ given in table 12 , where the last column gives $P_{a}$ for $B_{6}^{(1 / 2)}(\mu)=0.57$ and $B_{8}^{(3 / 2)}(\mu)=0.76$. For this purpose $\mu_{\mathrm{EW}}=M_{W}, \mu_{b}=m_{b}\left(m_{b}\right), \mu_{c}=1.3 \mathrm{GeV}$ and $\mu=$ $1.53 \mathrm{GeV}$ have been used. The central value of the SM prediction is $\left(\varepsilon^{\prime} / \varepsilon\right)_{\mathrm{SM}}=1.5 \times 10^{-4}$ compared to $1.9 \times 10^{-4}$ in [20] due to different numerical inputs.

\section{Statistical approach and numerical input}

The input quantities included in our analysis are collected in table 13 and table 14 . The CKM parameters have to be determined independently of contributions from the VLQs. The "tree-level" fit carried out by the CKMfitter collaboration achieves such a determination, taking only measurements into account that are unaffected in our NP scenarios, i.e. (semi-)leptonic tree-level decays, tree-level determinations of $\gamma$ and $B \rightarrow \pi \pi, \pi \rho, \rho \rho$, used as a constraint on $\gamma$. The results of this fit are again quoted in table 13 .

As a statistical procedure, we choose a frequentist approach. The fits include as parameters of interest the VLQ couplings and in addition nuisance parameters, which constitute theoretical uncertainties. The nuisance parameters are listed in table 13 and consist of

- CKM parameters from a "tree-level" fit; ${ }^{18}$

- hadronic parameters: decay constants, form factors, $|\Delta F|=2$ hadronic matrix elements.

The 1- and 2-dimensional confidence regions (CL) of parameters are obtained by profiling over the remaining parameters, i.e. maximisation of the likelihood function over the subspace of remaining parameters for a fixed value of the (pair of) parameter(s) of interest. Similarly, correlation plots for pairs of observables are obtained by profiling over all parameters and imposing in addition the specific values for the pair observables. The 2-dimensional $68 \%$ and $95 \%$ confidence regions are determined then for two degrees of freedom. The SM predictions of observables are found in the same way by setting VLQ contributions to zero and profiling only over the CKM and hadronic nuisance parameters.

\footnotetext{
${ }^{18}$ We thank Sebastien Descotes-Genon for providing us an update of a tree-level CKM fit from CKMfitter [94].
} 


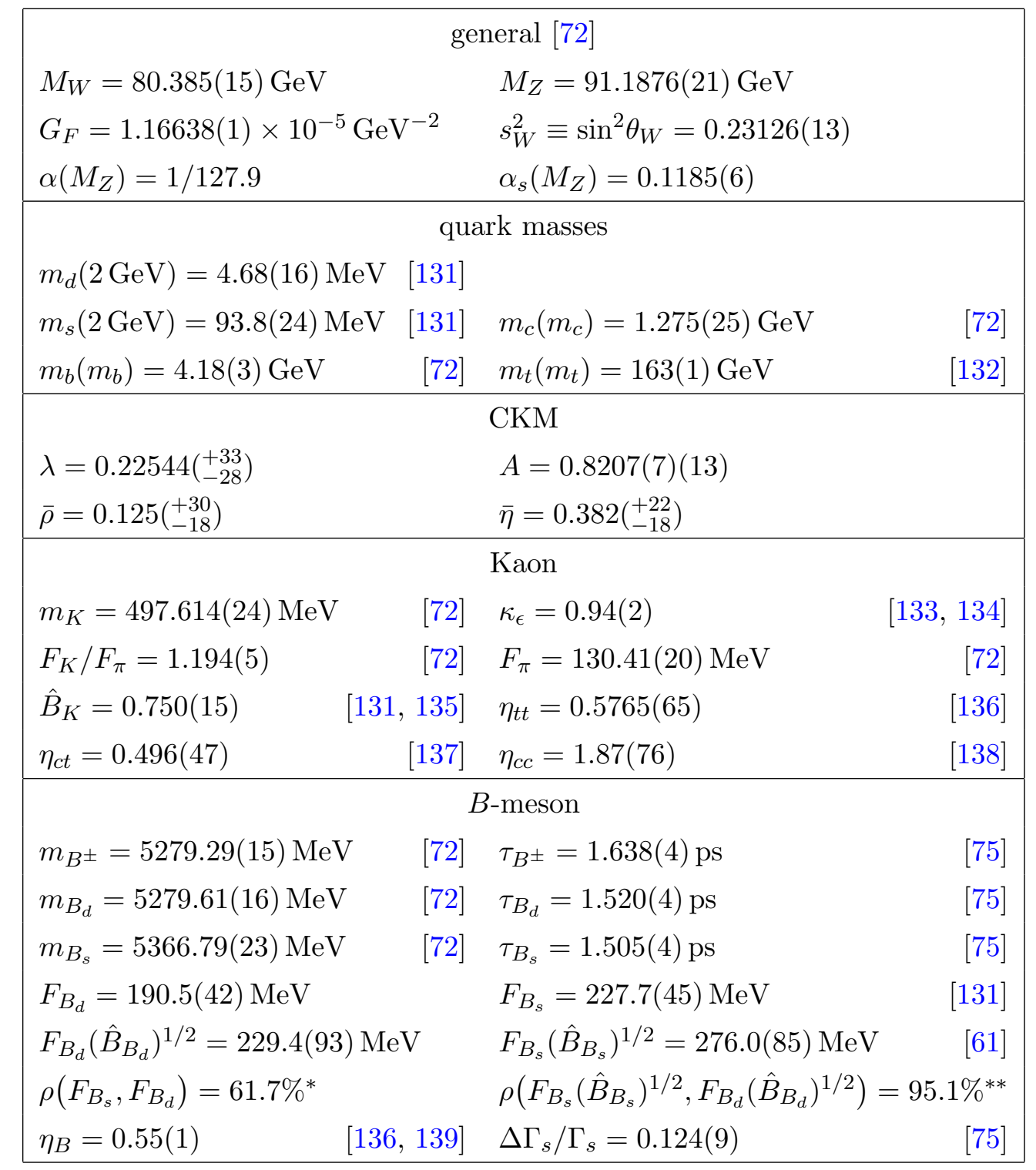

Table 13. Values of the experimental and theoretical quantities used as input parameters as of March 2016. ${ }^{*}$ : Calculated by demanding that the uncertainty of the ratio of the decay constants given above should equal the uncertainty given explicitly for the ratio, also given in ref. [131]. ${ }^{* *}$ : Calculated from information given in ref. [61]. Note that their assumption for the $\mathrm{SU}(3)$ breaking from the charm sea contribution corresponds to the assumption of a $91.8 \%$ correlation for this uncertainty between $B_{d}$ and $B_{s}$. 


\begin{tabular}{|c|cc|c|ccc|}
\hline$i j$ & $\mu_{\text {low }}[\mathrm{GeV}]$ & $N_{f}$ & $r_{\chi}$ & $B_{1}^{i j}$ & $B_{4}^{i j}$ & $B_{5}^{i j}$ \\
\hline$s d$ & 3.0 & 3 & 30.8 & $0.525(16)$ & $0.920(20)$ & $0.707(45)$ \\
\hline & & & & $F_{B_{j}}^{2} B_{1}^{i j}$ & $F_{B_{j}}^{2} B_{4}^{i j}$ & $F_{B_{j}}^{2} B_{5}^{i j}$ \\
\hline$b d$ & 4.18 & 5 & 1.6 & $0.0342(30)$ & $0.0390(29)$ & $0.0361(36)$ \\
$b s$ & 4.18 & 5 & 1.6 & $0.0498(32)$ & $0.0534(32)$ & $0.0493(37)$ \\
\hline
\end{tabular}

Table 14. Scale settings and number of flavours, $N_{f}$, as well as numerical inputs of bag factors entering $M_{12}^{i j}$, see [61] and [140] for correlations. For the Kaon system threshold crossings to $N_{f}=4$ and $N_{f}=3$ have been chosen as $4.18 \mathrm{GeV}$ and $1.4 \mathrm{GeV}$. The chirality-factor is given as $r_{\chi}^{i j}=\left(M_{M_{i j}} /\left(m_{i}\left(\mu_{\text {low }}\right)+m_{j}\left(\mu_{\text {low }}\right)\right)^{2}\right.$. See also [18] for more details on $M_{12}^{i j}$.

Open Access. This article is distributed under the terms of the Creative Commons Attribution License (CC-BY 4.0), which permits any use, distribution and reproduction in any medium, provided the original author(s) and source are credited.

\section{References}

[1] Y. Nir and D.J. Silverman, $Z$ mediated flavor changing neutral currents and their implications for CP asymmetries in $B^{0}$ decays, Phys. Rev. D 42 (1990) 1477 [INSPIRE].

[2] G.C. Branco, T. Morozumi, P.A. Parada and M.N. Rebelo, $C P$ asymmetries in $B^{0}$ decays in the presence of flavor changing neutral currents, Phys. Rev. D 48 (1993) 1167 [INSPIRE].

[3] F. del Aguila, M. Pérez-Victoria and J. Santiago, Observable contributions of new exotic quarks to quark mixing, JHEP 09 (2000) 011 [hep-ph/0007316] [INSPIRE].

[4] G. Barenboim, F.J. Botella and O. Vives, Constraining models with vector-like fermions from FCNC in K and B physics, Nucl. Phys. B 613 (2001) 285 [hep-ph/0105306] [INSPIRE].

[5] A.J. Buras, B. Duling and S. Gori, The impact of Kaluza-Klein fermions on Standard Model fermion couplings in a RS model with custodial protection, JHEP 09 (2009) 076 [arXiv:0905.2318] [INSPIRE].

[6] F.J. Botella, G.C. Branco and M. Nebot, The hunt for new physics in the flavour sector with up vector-like quarks, JHEP 12 (2012) 040 [arXiv:1207.4440] [INSPIRE].

[7] S. Fajfer, A. Greljo, J.F. Kamenik and I. Mustac, Light Higgs and vector-like quarks without prejudice, JHEP 07 (2013) 155 [arXiv: 1304.4219] [INSPIRE].

[8] A.J. Buras, J. Girrbach and R. Ziegler, Particle-antiparticle mixing, CP-violation and rare $K$ and $B$ decays in a minimal theory of fermion masses, JHEP 04 (2013) 168 [arXiv:1301.5498] [INSPIRE].

[9] W. Altmannshofer, S. Gori, M. Pospelov and I. Yavin, Quark flavor transitions in $L_{\mu}-L_{\tau}$ models, Phys. Rev. D 89 (2014) 095033 [arXiv: 1403.1269] [InSPIRE].

[10] A.K. Alok, S. Banerjee, D. Kumar, S.U. Sankar and D. London, New-physics signals of a model with a vector-singlet up-type quark, Phys. Rev. D 92 (2015) 013002 [arXiv: 1504.00517] [INSPIRE]. 
[11] K. Ishiwata, Z. Ligeti and M.B. Wise, New vector-like fermions and flavor physics, JHEP 10 (2015) 027 [arXiv: 1506.03484] [INSPIRE].

[12] P. Arnan, L. Hofer, F. Mescia and A. Crivellin, Loop effects of heavy new scalars and fermions in $b \rightarrow s \mu^{+} \mu^{-}$, arXiv:1608.07832 [INSPIRE].

[13] X.G. He, G.C. Joshi, H. Lew and R.R. Volkas, New Z' phenomenology, Phys. Rev. D 43 (1991) 22 [INSPIRE].

[14] X.-G. He, G.C. Joshi, H. Lew and R.R. Volkas, Simplest Z' model, Phys. Rev. D 44 (1991) 2118 [INSPIRE].

[15] W. Altmannshofer and I. Yavin, Predictions for lepton flavor universality violation in rare $B$ decays in models with gauged $L_{\mu}-L_{\tau}$, Phys. Rev. D 92 (2015) 075022 [arXiv: 1508.07009] [INSPIRE].

[16] A.J. Buras and J. Girrbach, Left-handed $Z^{\prime}$ and $Z$ FCNC quark couplings facing new $b \rightarrow s \mu^{+} \mu^{-}$data, JHEP 12 (2013) 009 [arXiv:1309.2466] [INSPIRE].

[17] F. del Aguila, J. de Blas and M. Pérez-Victoria, Effects of new leptons in electroweak precision data, Phys. Rev. D 78 (2008) 013010 [arXiv:0803.4008] [INSPIRE].

[18] C. Bobeth, A.J. Buras, A. Celis and M. Jung, Yukawa enhancement of Z-mediated new physics in $\Delta S=2$ and $\Delta B=2$ processes, arXiv:1703.04753 [INSPIRE].

[19] RBC-UKQCD collaboration, Z. Bai et al., Standard Model prediction for direct CP-violation in $K \rightarrow \pi \pi$ decay, Phys. Rev. Lett. 115 (2015) 212001 [arXiv:1505.07863] [INSPIRE].

[20] A.J. Buras, M. Gorbahn, S. Jäger and M. Jamin, Improved anatomy of $\epsilon^{\prime} / \epsilon$ in the Standard Model, JHEP 11 (2015) 202 [arXiv:1507.06345] [INSPIRE].

[21] A.J. Buras and J.-M. Gérard, Upper bounds on $\epsilon^{\prime} / \epsilon$ parameters $B_{6}^{(1 / 2)}$ and $B_{8}^{(3 / 2)}$ from large- $N$ QCD and other news, JHEP 12 (2015) 008 [arXiv:1507.06326] [INSPIRE].

[22] T. Kitahara, U. Nierste and P. Tremper, Singularity-free next-to-leading order $\Delta S=1$ renormalization group evolution and $\epsilon_{K}^{\prime} / \epsilon_{K}$ in the Standard Model and beyond, JHEP 12 (2016) 078 [arXiv: 1607.06727] [INSPIRE].

[23] A. Crivellin, G. D'Ambrosio and J. Heeck, Explaining $h \rightarrow \mu^{ \pm} \tau^{\mp}, B \rightarrow K^{*} \mu^{+} \mu^{-}$and $B \rightarrow K \mu^{+} \mu^{-} / B \rightarrow K e^{+} e^{-}$in a two-Higgs-doublet model with gauged $L_{\mu}-L_{\tau}$, Phys. Rev. Lett. 114 (2015) 151801 [arXiv:1501.00993] [INSPIRE].

[24] Sld Electroweak Group, Delphi, Aleph, SLD, SlD Heavy Flavour Group, OPAL, LEP EleCtroweAk Working Group and L3 collaborations, S. Schael et al., Precision electroweak measurements on the $Z$ resonance, Phys. Rept. 427 (2006) 257 [hep-ex/0509008] [INSPIRE].

[25] F. del Aguila, M. Chala, J. Santiago and Y. Yamamoto, Collider limits on leptophilic interactions, JHEP 03 (2015) 059 [arXiv:1411.7394] [INSPIRE].

[26] B. Grzadkowski, M. Iskrzynski, M. Misiak and J. Rosiek, Dimension-six terms in the Standard Model Lagrangian, JHEP 10 (2010) 085 [arXiv: 1008.4884] [INSPIRE].

[27] E.E. Jenkins, A.V. Manohar and M. Trott, Renormalization group evolution of the Standard Model dimension six operators I: formalism and $\lambda$ dependence, JHEP 10 (2013) 087 [arXiv: 1308.2627] [INSPIRE]. 
[28] E.E. Jenkins, A.V. Manohar and M. Trott, Renormalization group evolution of the Standard Model dimension six operators II: Yukawa dependence, JHEP 01 (2014) 035 [arXiv:1310.4838] [INSPIRE].

[29] R. Alonso, E.E. Jenkins, A.V. Manohar and M. Trott, Renormalization group evolution of the Standard Model dimension six operators III: gauge coupling dependence and phenomenology, JHEP 04 (2014) 159 [arXiv:1312.2014] [INSPIRE].

[30] C. Arzt, M.B. Einhorn and J. Wudka, Patterns of deviation from the Standard Model, Nucl. Phys. B 433 (1995) 41 [hep-ph/9405214] [InSPIRE].

[31] W. Buchmüller and D. Wyler, Effective Lagrangian analysis of new interactions and flavor conservation, Nucl. Phys. B 268 (1986) 621 [INSPIRE].

[32] J. Aebischer, A. Crivellin, M. Fael and C. Greub, Matching of gauge invariant dimension-six operators for $b \rightarrow s$ and $b \rightarrow c$ transitions, JHEP 05 (2016) 037 [arXiv: 1512.02830] [INSPIRE].

[33] M. Ciuchini, E. Franco, V. Lubicz, G. Martinelli, I. Scimemi and L. Silvestrini, Next-to-leading order QCD corrections to $\Delta F=2$ effective Hamiltonians, Nucl. Phys. B 523 (1998) 501 [hep-ph/9711402] [INSPIRE].

[34] A.J. Buras, M. Misiak and J. Urban, Two loop QCD anomalous dimensions of flavor changing four quark operators within and beyond the Standard Model, Nucl. Phys. B 586 (2000) 397 [hep-ph/0005183] [INSPIRE].

[35] A.J. Buras, F. De Fazio and J. Girrbach, The anatomy of $Z^{\prime}$ and $Z$ with flavour changing neutral currents in the flavour precision era, JHEP 02 (2013) 116 [arXiv:1211.1896] [INSPIRE].

[36] A.J. Buras, Minimal flavour violation and beyond: towards a flavour code for short distance dynamics, Acta Phys. Polon. B 41 (2010) 2487 [arXiv: 1012.1447] [INSPIRE].

[37] A.J. Buras, F. De Fazio, J. Girrbach and M.V. Carlucci, The anatomy of quark flavour observables in 331 models in the flavour precision era, JHEP 02 (2013) 023 [arXiv:1211.1237] [INSPIRE].

[38] A.J. Buras and J. Girrbach, Complete NLO QCD corrections for tree level $\triangle F=2 F C N C$ processes, JHEP 03 (2012) 052 [arXiv:1201.1302] [INSPIRE].

[39] C. Bobeth, M. Gorbahn and E. Stamou, Electroweak corrections to $B_{s, d} \rightarrow \ell^{+} \ell^{-}$, Phys. Rev. D 89 (2014) 034023 [arXiv:1311.1348] [INSPIRE].

[40] A.J. Buras, New physics patterns in $\epsilon^{\prime} / \epsilon$ and $\epsilon_{K}$ with implications for rare kaon decays and $\Delta M_{K}$, JHEP 04 (2016) 071 [arXiv:1601.00005] [INSPIRE].

[41] T. Blum et al., $K \rightarrow \pi \pi \Delta I=3 / 2$ decay amplitude in the continuum limit, Phys. Rev. D 91 (2015) 074502 [arXiv:1502.00263] [INSPIRE].

[42] NA48 collaboration, J.R. Batley et al., A precision measurement of direct CP-violation in the decay of neutral kaons into two pions, Phys. Lett. B 544 (2002) 97 [hep-ex/0208009] [INSPIRE].

[43] KTEV collaboration, A. Alavi-Harati et al., Measurements of direct CP-violation, CPT symmetry and other parameters in the neutral kaon system, Phys. Rev. D 67 (2003) 012005 [Erratum ibid. D 70 (2004) 079904] [hep-ex/0208007] [INSPIRE]. 
[44] KTEV collaboration, E. Abouzaid et al., Precise measurements of direct CP-violation, CPT symmetry and other parameters in the neutral kaon system, Phys. Rev. D 83 (2011) 092001 [arXiv:1011.0127] [INSPIRE].

[45] A.J. Buras and J.-M. Gérard, Final state interactions in $K \rightarrow \pi \pi$ decays: $\Delta I=1 / 2$ rule vs. $\epsilon^{\prime} / \epsilon$, Eur. Phys. J. C 77 (2017) 10 [arXiv:1603.05686] [InSPIRE].

[46] V. Antonelli, S. Bertolini, M. Fabbrichesi and E.I. Lashin, The $\Delta I=1 / 2$ selection rule, Nucl. Phys. B 469 (1996) 181 [hep-ph/9511341] [INSPIRE].

[47] S. Bertolini, J.O. Eeg and M. Fabbrichesi, A new estimate of $\epsilon^{\prime} / \epsilon$, Nucl. Phys. B 476 (1996) 225 [hep-ph/9512356] [INSPIRE].

[48] J.M. Frere, J. Galand, A. Le Yaouanc, L. Oliver, O. Pene and J.C. Raynal, $K^{0}-\bar{K}^{0}$ in the $\mathrm{SU}(2)_{L} \times \mathrm{SU}(2)_{R} \times \mathrm{U}(1)$ model of CP-violation, Phys. Rev. D 46 (1992) 337 [InSPIRE].

[49] E. Pallante and A. Pich, Strong enhancement of $\epsilon^{\prime} / \epsilon$ through final state interactions, Phys. Rev. Lett. 84 (2000) 2568 [hep-ph/9911233] [INSPIRE].

[50] E. Pallante and A. Pich, Final state interactions in kaon decays, Nucl. Phys. B 592 (2001) 294 [hep-ph/0007208] [INSPIRE].

[51] M. Buchler, G. Colangelo, J. Kambor and F. Orellana, A note on the dispersive treatment of $K \rightarrow \pi \pi$ with the kaon off-shell, Phys. Lett. B 521 (2001) 29 [hep-ph/0102289] [INSPIRE].

[52] M. Buchler, G. Colangelo, J. Kambor and F. Orellana, Dispersion relations and soft pion theorems for $K \rightarrow \pi \pi$, Phys. Lett. B 521 (2001) 22 [hep-ph/0102287] [INSPIRE].

[53] E. Pallante, A. Pich and I. Scimemi, The Standard Model prediction for $\epsilon^{\prime} / \epsilon$, Nucl. Phys. B 617 (2001) 441 [hep-ph/0105011] [INSPIRE].

[54] A.J. Buras, D. Buttazzo and R. Knegjens, $K \rightarrow \pi \nu \bar{\nu}$ and $\epsilon^{\prime} / \epsilon$ in simplified new physics models, JHEP 11 (2015) 166 [arXiv:1507.08672] [INSPIRE].

[55] M. Blanke, A.J. Buras and S. Recksiegel, Quark flavour observables in the littlest Higgs model with T-parity after LHC run 1, Eur. Phys. J. C 76 (2016) 182 [arXiv:1507.06316] [INSPIRE].

[56] A.J. Buras and F. De Fazio, $\epsilon^{\prime} / \epsilon$ in 331 models, JHEP 03 (2016) 010 [arXiv:1512.02869] [INSPIRE].

[57] A.J. Buras and F. De Fazio, 331 models facing the tensions in $\Delta F=2$ processes with the impact on $\epsilon^{\prime} / \epsilon, B_{s} \rightarrow \mu^{+} \mu^{-}$and $B \rightarrow K^{*} \mu^{+} \mu^{-}$, JHEP 08 (2016) 115 [arXiv: 1604.02344] [INSPIRE].

[58] M. Tanimoto and K. Yamamoto, Probing SUSY with 10 TeV stop mass in rare decays and CP-violation of kaon, Prog. Theor. Exp. Phys. 2016 (2016) 123B02 [arXiv:1603.07960] [INSPIRE].

[59] T. Kitahara, U. Nierste and P. Tremper, Supersymmetric explanation of CP-violation in $K \rightarrow \pi \pi$ decays, Phys. Rev. Lett. 117 (2016) 091802 [arXiv:1604.07400] [InSPIRE].

[60] M. Endo, S. Mishima, D. Ueda and K. Yamamoto, Chargino contributions in light of recent $\epsilon^{\prime} / \epsilon$, Phys. Lett. B 762 (2016) 493 [arXiv:1608.01444] [InSPIRE].

[61] Fermilab Lattice and MiLC collaborations, A. Bazavov et al., $B_{(s)}^{0}$-mixing matrix elements from lattice QCD for the Standard Model and beyond, Phys. Rev. D 93 (2016) 113016 [arXiv: 1602.03560] [INSPIRE]. 
[62] M. Blanke and A.J. Buras, Universal unitarity triangle 2016 and the tension between $\Delta M_{s, d}$ and $\epsilon_{K}$ in CMFV models, Eur. Phys. J. C 76 (2016) 197 [arXiv: 1602.04020] [INSPIRE].

[63] M. Endo, T. Kitahara, S. Mishima and K. Yamamoto, Revisiting kaon physics in general Z scenario, arXiv:1612.08839 [INSPIRE].

[64] G.C. Branco, P.M. Ferreira, L. Lavoura, M.N. Rebelo, M. Sher and J.P. Silva, Theory and phenomenology of two-Higgs-doublet models, Phys. Rept. 516 (2012) 1 [arXiv:1106.0034] [INSPIRE].

[65] A.J. Buras, F. De Fazio and J. Girrbach, 331 models facing new $b \rightarrow s \mu^{+} \mu^{-}$data, JHEP 02 (2014) 112 [arXiv: 1311.6729] [INSPIRE].

[66] A. Efrati, A. Falkowski and Y. Soreq, Electroweak constraints on flavorful effective theories, JHEP 07 (2015) 018 [arXiv:1503.07872] [INSPIRE].

[67] Z. Bai, N.H. Christ, T. Izubuchi, C.T. Sachrajda, A. Soni and J. Yu, $K_{L}-K_{S}$ mass difference from lattice QCD, Phys. Rev. Lett. 113 (2014) 112003 [arXiv:1406.0916] [INSPIRE].

[68] W. Altmannshofer and D.M. Straub, New physics in $b \rightarrow s$ transitions after LHC run 1 , Eur. Phys. J. C 75 (2015) 382 [arXiv:1411.3161] [InSPIRE].

[69] W. Altmannshofer and D.M. Straub, Implications of $b \rightarrow s$ measurements, arXiv: 1503.06199 [INSPIRE].

[70] S. Descotes-Genon, L. Hofer, J. Matias and J. Virto, Global analysis of $b \rightarrow$ sll anomalies, JHEP 06 (2016) 092 [arXiv: 1510.04239] [INSPIRE].

[71] T. Hurth, F. Mahmoudi and S. Neshatpour, On the anomalies in the latest LHCb data, Nucl. Phys. B 909 (2016) 737 [arXiv:1603.00865] [INSPIRE].

[72] Particle Data Group collaboration, K.A. Olive et al., Review of particle physics, Chin. Phys. C 38 (2014) 090001 [INSPIRE].

[73] E949 collaboration, A.V. Artamonov et al., New measurement of the $K^{+} \rightarrow \pi^{+} \nu \bar{\nu}$ branching ratio, Phys. Rev. Lett. 101 (2008) 191802 [arXiv:0808.2459] [INSPIRE].

[74] G. Isidori and R. Unterdorfer, On the short distance constraints from $K_{L, S} \rightarrow \mu^{+} \mu^{-}$, JHEP 01 (2004) 009 [hep-ph/0311084] [INSPIRE].

[75] Heavy Flavor Averaging Group (HFAG) collaboration, Y. Amhis et al., Averages of b-hadron, c-hadron and $\tau$-lepton properties as of summer 2014, arXiv:1412.7515 [INSPIRE].

[76] LHCb collaboration, First measurement of the differential branching fraction and CP asymmetry of the $B^{ \pm} \rightarrow \pi^{ \pm} \mu^{+} \mu^{-}$decay, JHEP 10 (2015) 034 [arXiv:1509.00414] [INSPIRE].

[77] LHCb and CMS collaborations, Observation of the rare $B_{s}^{0} \rightarrow \mu^{+} \mu^{-}$decay from the combined analysis of CMS and LHCb data, Nature $\mathbf{5 2 2}$ (2015) 68 [arXiv:1411.4413] [INSPIRE].

[78] LHCb collaboration, Differential branching fractions and isospin asymmetries of $B \rightarrow K^{(*)} \mu^{+} \mu^{-}$decays, JHEP 06 (2014) 133 [arXiv:1403.8044] [INSPIRE].

[79] M. Jung, Determining weak phases from $B \rightarrow J / \psi P$ decays, Phys. Rev. D 86 (2012) 053008 [arXiv: 1206. 2050] [INSPIRE].

[80] K. De Bruyn and R. Fleischer, A roadmap to control penguin effects in $B_{d}^{0} \rightarrow J / \psi K_{\mathrm{S}}^{0}$ and $B_{s}^{0} \rightarrow J / \psi \phi, J H E P 03$ (2015) 145 [arXiv:1412.6834] [INSPIRE]. 
[81] P. Frings, U. Nierste and M. Wiebusch, Penguin contributions to CP phases in $B_{d, s}$ decays to charmonium, Phys. Rev. Lett. 115 (2015) 061802 [arXiv:1503.00859] [INSPIRE].

[82] F. Newson et al., Prospects for $K^{+} \rightarrow \pi^{+} \nu \bar{\nu}$ at CERN in NA62, arXiv:1411.0109 [INSPIRE].

[83] A. Ceccucci, Direct CP-violation and rare $K$ decays perspectives, Acta Phys. Polon. B 47 (2016) 261 [INSPIRE].

[84] E391A collaboration, J.K. Ahn et al., Experimental study of the decay $K_{L}^{0} \rightarrow \pi^{0} \nu \bar{\nu}$, Phys. Rev. D 81 (2010) 072004 [arXiv:0911.4789] [INSPIRE].

[85] LHCb collaboration, Measurement of the $B_{s}^{0} \rightarrow \mu^{+} \mu^{-}$branching fraction and search for $B^{0} \rightarrow \mu^{+} \mu^{-}$decays at the LHCb experiment, Phys. Rev. Lett. 111 (2013) 101805 [arXiv: 1307.5024] [INSPIRE].

[86] LHCb collaboration, Angular analysis of the $B^{0} \rightarrow K^{* 0} \mu^{+} \mu^{-}$decay using $3 \mathrm{fb}^{-1}$ of integrated luminosity, JHEP 02 (2016) 104 [arXiv:1512.04442] [INSPIRE].

[87] BABAR collaboration, J.P. Lees et al., Search for $B \rightarrow K^{(*)} \nu \bar{\nu}$ and invisible quarkonium decays, Phys. Rev. D 87 (2013) 112005 [arXiv: 1303.7465] [inSPIRE].

[88] Belle collaboration, O. Lutz et al., Search for $B \rightarrow h^{(*)} \nu \bar{\nu}$ with the full Belle $\Upsilon(4 S)$ data sample, Phys. Rev. D 87 (2013) 111103 [arXiv:1303.3719] [INSPIRE].

[89] C. Bobeth, G. Hiller and G. Piranishvili, CP asymmetries in $\bar{B} \rightarrow \bar{K}^{*}(\rightarrow \bar{K} \pi) \bar{\ell} \ell$ and untagged $\bar{B}_{s}, B_{s} \rightarrow \phi\left(\rightarrow K^{+} K^{-}\right) \overline{\ell \ell}$ decays at NLO, JHEP 07 (2008) 106 [arXiv:0805.2525] [INSPIRE].

[90] C. Bobeth, G. Hiller and D. van Dyk, General analysis of $\bar{B} \rightarrow \bar{K}^{(*)} \ell^{+} \ell^{-}$decays at low recoil, Phys. Rev. D 87 (2013) 034016 [arXiv:1212.2321] [INSPIRE].

[91] W. Altmannshofer, A.J. Buras, D.M. Straub and M. Wick, New strategies for new physics search in $B \rightarrow K^{*} \nu \bar{\nu}, B \rightarrow K \nu \bar{\nu}$ and $B \rightarrow X_{s} \nu \bar{\nu}$ decays, JHEP 04 (2009) 022 [arXiv: 0902.0160] [INSPIRE].

[92] A.J. Buras, J. Girrbach-Noe, C. Niehoff and D.M. Straub, $B \rightarrow K^{(*)} \nu \bar{\nu}$ decays in the Standard Model and beyond, JHEP 02 (2015) 184 [arXiv: 1409.4557] [INSPIRE].

[93] T. Aushev et al., Physics at super B factory, arXiv:1002.5012 [INSPIRE].

[94] CKMfitter Group collaboration, J. Charles et al., CP violation and the CKM matrix: assessing the impact of the asymmetric B factories, Eur. Phys. J. C 41 (2005) 1 [hep-ph/0406184] [INSPIRE].

[95] L. Basso, S. Moretti and G.M. Pruna, A renormalisation group equation study of the scalar sector of the minimal B-L extension of the Standard Model, Phys. Rev. D 82 (2010) 055018 [arXiv: 1004.3039] [INSPIRE].

[96] K.S. Babu, C.F. Kolda and J. March-Russell, Implications of generalized Z-Z' mixing, Phys. Rev. D 57 (1998) 6788 [hep-ph/9710441] [INSPIRE].

[97] A.J. Buras, S. Jager and J. Urban, Master formulae for $\Delta F=2 N L O Q C D$ factors in the Standard Model and beyond, Nucl. Phys. B 605 (2001) 600 [hep-ph/0102316] [InSPIRE].

[98] M. Gorbahn, S. Jager, U. Nierste and S. Trine, The supersymmetric Higgs sector and B- $\bar{B}$ mixing for large $\tan \beta$, Phys. Rev. D 84 (2011) 034030 [arXiv:0901.2065] [INSPIRE].

[99] G. Buchalla, A.J. Buras and M.E. Lautenbacher, Weak decays beyond leading logarithms, Rev. Mod. Phys. 68 (1996) 1125 [hep-ph/9512380] [INSPIRE]. 
[100] A.J. Buras, Weak Hamiltonian, CP-violation and rare decays, hep-ph/9806471 [INSPIRE].

[101] T. Inami and C.S. Lim, Effects of superheavy quarks and leptons in low-energy weak processes $K_{L} \rightarrow \mu \bar{\mu}, K^{+} \rightarrow \pi^{+} \nu \bar{\nu}$ and $K^{0} \rightarrow \bar{K}^{0}$, Prog. Theor. Phys. 65 (1981) 297 [Erratum ibid. 65 (1981) 1772][INSPIRE].

[102] G. Buchalla, A.J. Buras and M.K. Harlander, Penguin box expansion: flavor changing neutral current processes and a heavy top quark, Nucl. Phys. B 349 (1991) 1 [INSPIRE].

[103] G. Buchalla and A.J. Buras, QCD corrections to rare $K$ and $B$ decays for arbitrary top quark mass, Nucl. Phys. B 400 (1993) 225 [INSPIRE].

[104] M. Misiak and J. Urban, QCD corrections to FCNC decays mediated by $Z$ penguins and $W$ boxes, Phys. Lett. B 451 (1999) 161 [hep-ph/9901278] [INSPIRE].

[105] G. Buchalla and A.J. Buras, The rare decays $K \rightarrow \pi \nu \bar{\nu}, B \rightarrow X \nu \bar{\nu}$ and $B \rightarrow \ell^{+} \ell^{-}$: an update, Nucl. Phys. B 548 (1999) 309 [hep-ph/9901288] [INSPIRE].

[106] J. Brod, M. Gorbahn and E. Stamou, Two-loop electroweak corrections for the $K \rightarrow \pi \nu \bar{\nu}$ decays, Phys. Rev. D 83 (2011) 034030 [arXiv: 1009.0947] [INSPIRE].

[107] A.J. Buras, D. Buttazzo, J. Girrbach-Noe and R. Knegjens, $K^{+} \rightarrow \pi^{+} \nu \bar{\nu}$ and $K_{L} \rightarrow \pi^{0} \nu \bar{\nu}$ in the Standard Model: status and perspectives, JHEP 11 (2015) 033 [arXiv:1503.02693] [INSPIRE].

[108] A.J. Buras, M. Gorbahn, U. Haisch and U. Nierste, Charm quark contribution to $K^{+} \rightarrow \pi^{+} \nu \bar{\nu}$ at next-to-next-to-leading order, JHEP 11 (2006) 002 [Erratum ibid. 11 (2012) 167] [hep-ph/0603079] [INSPIRE].

[109] G. Isidori, F. Mescia and C. Smith, Light-quark loops in $K \rightarrow \pi \nu \bar{\nu}$, Nucl. Phys. B 718 (2005) 319 [hep-ph/0503107] [INSPIRE].

[110] F. Mescia and C. Smith, Improved estimates of rare $K$ decay matrix-elements from $K_{\ell 3}$ decays, Phys. Rev. D 76 (2007) 034017 [arXiv: 0705. 2025] [INSPIRE].

[111] C. Bobeth, G. Hiller and G. Piranishvili, Angular distributions of $\bar{B} \rightarrow \bar{K} \ell^{+} \ell^{-}$decays, JHEP 12 (2007) 040 [arXiv:0709.4174] [INSPIRE].

[112] T. Hermann, M. Misiak and M. Steinhauser, Three-loop QCD corrections to $B_{s} \rightarrow \mu^{+} \mu^{-}$, JHEP 12 (2013) 097 [arXiv: 1311.1347] [INSPIRE].

[113] B. Grinstein and D. Pirjol, Exclusive rare $B \rightarrow K^{*} \ell^{+} \ell^{-}$decays at low recoil: controlling the long-distance effects, Phys. Rev. D 70 (2004) 114005 [hep-ph/0404250] [inSPIRE].

[114] M. Beylich, G. Buchalla and T. Feldmann, Theory of $B \rightarrow K^{(*)} \ell^{+} \ell^{-}$decays at high $q^{2}$ : OPE and quark-hadron duality, Eur. Phys. J. C 71 (2011) 1635 [arXiv:1101.5118] [INSPIRE].

[115] C. Bobeth, G. Hiller, D. van Dyk and C. Wacker, The decay $B \rightarrow K \ell^{+} \ell^{-}$at low hadronic recoil and model-independent $\Delta B=1$ constraints, JHEP 01 (2012) 107 [arXiv:1111.2558] [INSPIRE].

[116] D. Seidel, Analytic two loop virtual corrections to $b \rightarrow d \ell^{+} \ell^{-}$, Phys. Rev. D 70 (2004) 094038 [hep-ph/0403185] [INSPIRE].

[117] C. Greub, V. Pilipp and C. Schupbach, Analytic calculation of two-loop QCD corrections to $b \rightarrow s \ell^{+} \ell^{-}$in the high $q^{2}$ region, JHEP 12 (2008) 040 [arXiv:0810.4077] [INSPIRE]. 
[118] Fermilab Lattice and MiLC collaborations, J.A. Bailey et al., $\left|V_{u b}\right|$ from $B \rightarrow \pi \ell \nu$ decays and $(2+1)$-flavor lattice QCD, Phys. Rev. D 92 (2015) 014024 [arXiv:1503.07839] [INSPIRE].

[119] Fermilab Lattice and MiLC collaborations, J.A. Bailey et al., $B \rightarrow \pi \ell \ell$ form factors for new-physics searches from lattice QCD, Phys. Rev. Lett. 115 (2015) 152002 [arXiv: 1507.01618] [INSPIRE].

[120] J.A. Bailey et al., $B \rightarrow K \ell^{+} \ell^{-}$decay form factors from three-flavor lattice $Q C D$, Phys. Rev. D 93 (2016) 025026 [arXiv: 1509.06235] [INSPIRE].

[121] D. Du et al., Phenomenology of semileptonic B-meson decays with form factors from lattice QCD, Phys. Rev. D 93 (2016) 034005 [arXiv: 1510.02349] [inSPIRE].

[122] C. Bobeth, G. Hiller and D. van Dyk, The benefits of $\bar{B} \rightarrow \bar{K}^{*} \ell^{+} \ell^{-}$decays at low recoil, JHEP 07 (2010) 098 [arXiv: 1006.5013] [InSPIRE].

[123] A. Bharucha, D.M. Straub and R. Zwicky, $B \rightarrow V \ell^{+} \ell^{-}$in the Standard Model from light-cone sum rules, JHEP 08 (2016) 098 [arXiv: 1503.05534] [INSPIRE].

[124] R.R. Horgan, Z. Liu, S. Meinel and M. Wingate, Lattice QCD calculation of form factors describing the rare decays $B \rightarrow K^{*} \ell^{+} \ell^{-}$and $B_{s} \rightarrow \phi \ell^{+} \ell^{-}$, Phys. Rev. D 89 (2014) 094501 [arXiv: 1310.3722$]$ [INSPIRE].

[125] R.R. Horgan, Z. Liu, S. Meinel and M. Wingate, Rare B decays using lattice QCD form factors, PoS (LATTICE2014) 372 [arXiv: 1501.00367] [INSPIRE].

[126] G. D'Ambrosio and J. Portoles, Vector meson exchange contributions to $K \rightarrow \pi \gamma \gamma$ and $K_{L} \rightarrow \gamma \ell^{+} \ell^{-}$, Nucl. Phys. B 492 (1997) 417 [hep-ph/9610244] [INSPIRE].

[127] D. Gomez Dumm and A. Pich, Long distance contributions to the $K_{L} \rightarrow \mu^{+} \mu^{-}$decay width, Phys. Rev. Lett. 80 (1998) 4633 [hep-ph/9801298] [INSPIRE].

[128] J.-M. Gérard, C. Smith and S. Trine, Radiative kaon decays and the penguin contribution to the $\Delta I=1 / 2$ rule, Nucl. Phys. B 730 (2005) 1 [hep-ph/0508189] [INSPIRE].

[129] A.J. Buras, M. Jamin and M.E. Lautenbacher, The anatomy of $\epsilon^{\prime} / \epsilon$ beyond leading logarithms with improved hadronic matrix elements, Nucl. Phys. B 408 (1993) 209 [hep-ph/9303284] [INSPIRE].

[130] A.L. Kagan, Right-handed currents, $C P$-violation and $B \rightarrow V V$, hep-ph/0407076 [INSPIRE].

[131] S. Aoki et al., Review of lattice results concerning low-energy particle physics, Eur. Phys. J. C 74 (2014) 2890 [arXiv:1310.8555] [INSPIRE].

[132] HPQCD collaboration, I. Allison et al., High-precision charm-quark mass from current-current correlators in lattice and continuum QCD, Phys. Rev. D 78 (2008) 054513 [arXiv: 0805.2999] [INSPIRE].

[133] A.J. Buras and D. Guadagnoli, Correlations among new CP-violating effects in $\Delta F=2$ observables, Phys. Rev. D 78 (2008) 033005 [arXiv: 0805.3887] [inSPIRE].

[134] A.J. Buras, D. Guadagnoli and G. Isidori, On $\epsilon_{K}$ beyond lowest order in the operator product expansion, Phys. Lett. B 688 (2010) 309 [arXiv:1002.3612] [INSPIRE].

[135] A.J. Buras, J.-M. Gérard and W.A. Bardeen, Large- $N$ approach to kaon decays and mixing 28 years later: $\Delta I=1 / 2$ rule, $\hat{B}_{K}$ and $\Delta M_{K}$, Eur. Phys. J. C 74 (2014) 2871 [arXiv:1401.1385] [INSPIRE]. 
[136] A.J. Buras, M. Jamin and P.H. Weisz, Leading and next-to-leading QCD corrections to $\epsilon$ parameter and $B^{0}-\bar{B}^{0}$ mixing in the presence of a heavy top quark, Nucl. Phys. B 347 (1990) 491 [INSPIRE].

[137] J. Brod and M. Gorbahn, $\epsilon_{K}$ at next-to-next-to-leading order: the charm-top-quark contribution, Phys. Rev. D 82 (2010) 094026 [arXiv: 1007.0684] [INSPIRE].

[138] J. Brod and M. Gorbahn, Next-to-next-to-leading-order charm-quark contribution to the CP-violation parameter $\epsilon_{K}$ and $\Delta M_{K}$, Phys. Rev. Lett. 108 (2012) 121801 [arXiv:1108.2036] [INSPIRE].

[139] J. Urban, F. Krauss, U. Jentschura and G. Soff, Next-to-leading order QCD corrections for the $B^{0}-\bar{B}^{0}$ mixing with an extended Higgs sector, Nucl. Phys. B 523 (1998) 40 [hep-ph/9710245] [INSPIRE].

[140] RBC-UKQCD collaboration, N. Garron, R.J. Hudspith and A.T. Lytle, Neutral kaon mixing beyond the Standard Model with $n_{f}=2+1$ chiral fermions part 1: bare matrix elements and physical results, JHEP 11 (2016) 001 [arXiv: 1609.03334] [INSPIRE]. 